\title{
TRANSONIC SHOCK SOLUTIONS FOR A SYSTEM OF EULER-POISSON EQUATIONS*
}

\author{
TAO $\mathrm{LUO}^{\dagger}$ AND ZHOUPING XIN $\ddagger$
}

\begin{abstract}
A boundary value problem for a system of Euler-Poisson equations modeling semiconductor devices or plasma is considered. The boundary conditions are supersonic inflow and subsonic outflow. The purpose of this paper is to elucidate the role played by the electric field in the structure of solutions with transonic shocks. The existence, non-existence, uniqueness, and non-uniqueness of solutions with transonic shocks are obtained according to the different cases of boundary data and physical interval length. Detailed structures of solutions are given. Shock locations are determined by the boundary data. Different phenomena are shown for the different situations when the density of fixed, positively charged background ions is in supersonic and subsonic regimes.
\end{abstract}

Key words. Euler-Poisson equations, transonic shocks.

AMS subject classifications. 35L67, 35L65, 35Q35.

\section{Introduction}

The following system of one-dimensional Euler-Poisson equations:

$$
\left\{\begin{array}{l}
\rho_{t}+(\rho u)_{x}=0, \\
(\rho u)_{t}+\left(p(\rho)+\rho u^{2}\right)_{x}=\rho E, \\
E_{x}=\rho-b
\end{array}\right.
$$

where $x$ and $t$ are space and time variables, respectively, models several physical flows including the propagation of electrons in submicron semiconductor devices and plasma (cf. [13]) (hydrodynamic model), and the biological transport of ions for channel proteins (cf. [2]). In the hydrodynamical model of semiconductor devices or plasma, $u, \rho$, and $p$ represent the average particle velocity, electron density, and pressure, respectively, $E$ is the electric field, which is generated by the Coulomb force of particles, and $b>0$ stands for the density of fixed, positively charged background ions. The biological model describes the transport of ions between the extracellular side and the cytoplasmic side of the membranes ([2]). In this case, $\rho, \rho u$, and $E$ are the ion concentration, the ions translational mass, and the electric field, respectively.

In this paper, we study the transonic shock solutions for the following timeindependent problem:

$$
(\rho u)_{x}=0,\left(p(\rho)+\rho u^{2}\right)_{x}=\rho E, E_{x}=\rho-b,
$$

Assume that $p$ satisfies

$$
p(0)=0, p^{\prime}(\rho)>0, p^{\prime \prime}(\rho)>0, \text { for } \rho>0, p(+\infty)=+\infty .
$$

We consider a boundary value problem for (1.2) in an interval $0 \leq x \leq L$ with the boundary conditions

$$
(\rho, u, E)(0)=\left(\rho_{l}, u_{l}, \alpha\right), \quad(\rho, u)(L)=\left(\rho_{r}, u_{r}\right) .
$$

\footnotetext{
*Received: April 16, 2011; accepted: June 24, 2011. Communicated by Alberto Bressan.

${ }^{\dagger}$ Department of Mathematics and Statistics, Georgetown University, Box 571233 St. Mary's Hall 338A, Washington, DC 20057-1233, USA (t148@georgetown.edu).

$\ddagger$ The Institute of Mathematical Sciences and Department of Mathematics, Room 701 Academic Building No.1, The Chinese University of Hong Kong, Shatin, Hong Kong (zpxin@ims.cuhk.edu.hk).
} 
It will assumed that $u_{l}>0$ and $u_{r}>0$. It follows from the first equation in (1.2) that $\rho u(x)=$ constant $(0 \leq x \leq L)$ so the boundary data should satisfy $\rho_{l} u_{l}=\rho_{r} u_{r}$. We denote

$$
\rho_{l} u_{l}=\rho_{r} u_{r}=J .
$$

Then $\rho u(x)=J(0 \leq x \leq L)$ and the velocity is given by

$$
u=J / \rho .
$$

The boundary value problem for system (1.2) reduces to

$$
\left(p(\rho)+\frac{J^{2}}{\rho}\right)_{x}=\rho E, \quad E_{x}=\rho-b,
$$

with the boundary conditions

$$
(\rho, E)(0)=\left(\rho_{l}, \alpha\right), \quad \rho(L)=\rho_{r} .
$$

We use the terminology from gas dynamics to call $c=\sqrt{p^{\prime}(\rho)}$ the sound speed. There is a unique solution $\rho=\rho_{s}$ for the equation $p^{\prime}(\rho) \rho^{2}=J^{2}$, which is the sonic state (recall that $J=\rho u)$. In this case, the flow is called supersonic if

$$
p^{\prime}(\rho) \rho^{2}<J^{2} \text {, i.e., } \rho<\rho_{s} .
$$

If

$$
p^{\prime}(\rho) \rho^{2}>J^{2} \text {, i.e., } \rho>\rho_{s},
$$

then the flow is called subsonic.

We notice that $(1.7)_{1}$ is singular at sonic state $\left(p^{\prime}\left(\rho_{s}\right)-\frac{J^{2}}{\rho_{s}^{2}}=0\right)$ and the coefficient of $\rho_{x}$ changes the sign for the supersonic flow and subsonic flow. This makes the problem of determining which kind of boundary conditions should be posed to make the boundary value problem well-posed a subtle one. In previous works, some pure subsonic or supersonic solutions are obtained for both one-dimensional and multidimensional cases (cf. [5] and [13]). For a viscous approximation of transonic solutions in the two-dimensional case for the equations of semiconductors, see [7]. However, there have been only a few results for the transonic flow. In the following, we list several results which are closely related to the present paper. First, a boundary value problem for (1.7) was discussed in [1] for a linear pressure function of the form $p(\rho)=k \rho$ with the special boundary conditions $\rho(0)=\rho(L)=\bar{\rho}$ with $\bar{\rho}$ being a subsonic state for the case when $0<b<\rho_{s}$. The solution obtained in [1] may contain transonic shock. On the other hand, since the boundary conditions and the pressure function are special in [1], it is desirable to consider the more general boundary conditions with a more general pressure function. Moreover, only the case when $0<b<\rho_{s}$ (i.e., when $b$ is in the supersonic regime) is considered. As we will show later, the cases when $0<b<\rho_{s}$ and $b>\rho_{s}$ are completely different. Actually, $(b, 0)$ is a center when $0<b<\rho_{s}$ and a saddle point when $b>\rho_{s}$ for system (1.7). We will construct solutions with transonic shocks for both cases. In [14], the local-in-time stability of transonic shock solutions for the Cauchy problem of (1.1) is considered by assuming the existence of steady transonic shocks. In [15], a phase plane analysis is given for system (1.7). However, no transonic shock solutions are constructed in [15]. A transonic solution which may 
contain transonic shocks was constructed by I. Gamba (cf. [8]) by using a vanishing viscosity limit method. However, the solutions as the limit of vanishing viscosity may contain boundary layers. Therefore, the question of well-posedness of the boundary value problem for the inviscid problem can not be answered by the vanishing viscosity method. Moreover, the structure of the solutions constructed by the vanishing viscosity method in [8] is shown to be of bounded total variation and possibly contain more than one transonic shocks. One of the main purposes of the present paper is to obtain more detailed structure of the solutions for the boundary value problem (1.7) and (1.8) and answer the question of well-posedness of solutions for this boundary value problem. We give a thorough study of the structure of the solutions to the boundary value problem for the different situations of boundary data and the interval length $L$. The existence, non-existence, uniqueness, and non-uniqueness of solutions with transonic shocks are obtained according to the different cases of boundary data and physical interval length. The solution (when it exists) that we construct contains exactly one transonic shock in the interval $[0, L]$. On the left of this transonic shock, the flow is supersonic, it is subsonic on the right of this shock. Moreover, we can determine the shock location by the boundary data and $L$. It is interesting to compare this result with the transonic solutions of a quasi-one-dimensional gas flow through a nozzle studied by Embid, Goodman, and Majda ([4]). The time-dependent equations for the one dimensional isentropic nozzle flow are

$$
\left\{\begin{array}{l}
\rho_{t}+(\rho u)_{x}=-\frac{A^{\prime}(x)}{A(x)} \rho u, \\
(\rho u)_{t}+\left(\rho u^{2}+p(\rho)\right)_{x}=-\frac{A^{\prime}(x)}{A(x)} \rho u^{2}
\end{array}\right.
$$

where $\rho, u$, and $p$ denote respectively the density, velocity, and pressure, and $A(x)$ is the cross-sectional area of the nozzle. In [4], steady state solutions containing transonic shocks are constructed for the boundary value problem in the interval $[0$, $1]$ with the boundary conditions $(\rho, u)(0)=\left(\rho_{l}, u_{l}\right)$ and $(\rho, u)(1)=\left(\rho_{r}, u_{r}\right)$ satisfying $\rho_{l} u_{l}=\rho_{r} u_{r}$, with $\left(\rho_{l}, u_{l}\right)$ being supersonic and $\left(\rho_{r}, u_{r}\right)$ being subsonic. It is shown in [4] that, if $A(x)$ is not strictly monotone, then there exist multiple steady state transonic shock solutions, and the shock locations are not unique. Particularly, when $A^{\prime}(x) \equiv 0$ (this means the duct is uniform), the transonic shock can be anywhere in the duct. Therefore, the structure of solutions depends on the geometry of the nozzle. The electric field $E$ plays a similar role as we will show later. The difference is that the geometry of the nozzle is given, while the electric field $E$ is unknown and is a part of the solutions.

There have been many studies on the stability of transonic shocks for system (1.13) (cf. [10], [11], and [9]). It is interesting to investigate the stability of steady transonic solutions obtained in the paper. This is achieved recently in [12]. It would be interesting to extend the results of this paper to the multi-dimensional case, as those for gas dynamics (cf. [3] and [16]). An effort in this direction was made in [7] for a viscous approximation of transonic solutions in the 2-d case for the equations of semiconductors. However, passing to limit when the viscosity tends to zero for the viscosity approximation in [7] is still an open problem.

\section{Transonic shock solutions}

For the boundary value problem (1.7) and (1.8), we assume that $\rho_{l}<\rho_{s}$ and $\rho_{r}>\rho_{s}$. This means that the flow is supersonic at $x=0$ and subsonic at $x=L$. The solutions for this boundary value problem are expected to have transonic shocks in the interval $[0, L]$. A transonic shock solution is a discontinuous solution of the boundary 
value problem (1.7) and (1.8). Suppose the shock location is at a point $a \in[0, L]$, then we require the following Rankine-Hugoniot condition and entropy condition: Rankine-Hugoniot condition

$$
\left(p(\rho)+\frac{J^{2}}{\rho}\right)(a+)=\left(p(\rho)+\frac{J^{2}}{\rho}\right)(a-), E(a+)=E(a-) ;
$$

\section{Entropy condition}

$$
\rho(a+)>\rho(a-) .
$$

To say that the shock is transonic means

$$
\rho(a+)>\rho_{s}>\rho(a-) .
$$

The definition of transonic shock solutions for the boundary value problem (1.7) and (1.8) is given as follows:

Definition 2.1. A piecewise smooth solution $(\rho, E)$ with $\rho>0$ to the boundary value problem (1.7) and (1.8) is said to be a transonic shock solution if it is separated by a shock discontinuity located at $a \in[0, L]$, and of the form

$$
(\rho, E)=\left\{\begin{array}{l}
\left(\rho_{\text {sup }}, E_{\text {sup }}\right)(x), 0 \leq x \leq a, \\
\left(\rho_{\text {sub }}, E_{\text {sub }}\right)(x), a \leq x \leq L,
\end{array}\right.
$$

satisfying the Rankine-Hugoniot condition

$$
p\left(\rho_{\text {sup }}(a)\right)+\frac{J^{2}}{\rho_{\text {sup }}(a)}=p\left(\rho_{\text {sub }}(a)\right)+\frac{J^{2}}{\rho_{\text {sub }}(a)}, E_{\text {sup }}(a)=E_{\text {sub }}(a),
$$

and is supersonic behind the shock and subsonic ahead of the shock, i.e.,

$$
\rho_{\text {sup }}(x)<\rho_{s} \text { for } x \in[0, a]
$$

and

$$
\rho_{\text {sub }}(x)>\rho_{s}, \text { for } x \in[a, L] .
$$

Moreover, $\left(\rho_{\text {sup }}, E_{\text {sup }}\right)(x)$ and $\left(\rho_{\text {sub }}, E_{\text {sub }}\right)(x)$ satisfy Equation (1.7) on the intervals $[0, a]$ and $[a, L]$, respectively, and the following boundary conditions:

$$
\left(\rho_{\text {sup }}, E_{\text {sup }}\right)(0)=\left(\rho_{l}, \alpha\right), \rho_{\text {sub }}(L)=\rho_{r} .
$$

The main result of this paper can be summarized as follows:

Proposition 2.2. For the cases of $b<\rho_{s}$ and $b>\rho_{s}$, one can classify the boundary data $\left(\rho_{l}, \alpha\right), \rho_{r}$, and the length of the interval $L$ such that the boundary value problem (1.7) and (1.8) has:

1) no transonic shock solution;

2) a unique transonic shock solution;

3) more than one transonic shock solution. 


\section{Initial value problem for system (1.7)}

We will use the shock matching method to construct the transonic shock solutions for the boundary value problem. For this purpose, we study the following initial value problem for (1.7):

$$
\left\{\begin{array}{l}
\left(p(\rho)+\frac{J^{2}}{\rho}\right)_{x}=\rho E, \quad E_{x}=\rho-b, \text { for } x>x_{0} \\
(\rho, E)\left(x_{0}\right)=\left(\rho_{0}, E_{0}\right) .
\end{array}\right.
$$

The solution of (1.7) can be analyzed in $(\rho, E)$-phase plane.

Any trajectory in $(\rho, E)$-plane satisfies the following equation:

$$
d\left(\frac{1}{2} E^{2}-H(\rho)\right)=0, \text { where } H^{\prime}(\rho)=\frac{\rho-b}{\rho}\left(p^{\prime}(\rho)-\frac{J^{2}}{\rho^{2}}\right) .
$$

The trajectory passing through the point $\left(\rho_{0}, E_{0}\right)$ with $\rho_{0}>0$ is given by

$$
\frac{1}{2} E^{2}-\int_{\rho_{0}}^{\rho} H^{\prime}(s) d s=\frac{1}{2} E_{0}^{2} .
$$

Since the cases when $0<b<\rho_{s}$ ( $b$ is in supersonic region) and $b>\rho_{s}$ ( $b$ is in subsonic region) are completely different, we discuss these two cases separately. The phase portraits for these two cases can be found in Figure 1 and Figure 2 (all the figures are at the end of this paper).

A phase plane analysis of Equation (1.7) is given in [15]. We give some details here for our study of the transonic shock solutions.

3.1. The case when $0<b<\rho_{s}$. The following facts will be useful:

$$
\begin{gathered}
H^{\prime}\left(\rho_{s}\right)=H^{\prime}(b)=0, H^{\prime}(\rho)>0 \text { for } 0<\rho<b \text { and } \rho>\rho_{s}, H^{\prime}(\rho)>0 \text { for } b<\rho<\rho_{s}, \\
\lim _{\rho \rightarrow 0^{+}} \int_{\rho_{0}}^{\rho} H^{\prime}(s) d s=-\infty \text { for any } \rho_{0}>0 .
\end{gathered}
$$

For the different situations of the initial value $\left(\rho_{0}, E_{0}\right)$ on the $(\rho, E)$-plane, we give the following classification of solutions. First, we define the critical trajectory for the case when $0<b<\rho_{s}$.

Definition 3.1. The critical trajectory is the trajectory passing through the point $\left(\rho_{s}, 0\right)$ with the equation

$$
\frac{1}{2} E^{2}-\int_{\rho_{s}}^{\rho} H^{\prime}(s) d s=0 .
$$

There are two branches of the critical trajectory - a supersonic branch and a subsonic branch.

The supersonic branch is for $\rho_{\min }^{c} \leq \rho \leq \rho_{s}$ where $\rho_{\min }^{c}$ is determined by

$$
\int_{\rho_{s}}^{\rho_{\min }^{c}} H^{\prime}(s) d s=0, \quad 0<\rho_{\min }^{c}<b .
$$

The subsonic branch is for $\rho>\rho_{s}$. The supersonic branch is a loop with center $(b, 0)$, which is called the supersonic loop of the critical trajectory. The supersonic branch and subsonic branch intersect at the sonic point $\left(\rho_{s}, 0\right)$. 
Solutions for IVP (3.1) for the case $0<b<\rho_{s}$.

Case 1: $\quad\left(\rho_{0}, E_{0}\right)$ is inside the critical supersonic loop, i.e., $\left(\frac{1}{2} E_{0}^{2}-\int_{\rho_{s}}^{\rho_{0}} H^{\prime}(s) d s<0\right.$ and $\left.0<\rho_{0}<\rho_{s}\left(\rho_{0}, E_{0}\right) \neq(b, 0)\right)$.

In this case, the initial value problem (3.1) admits a unique solution $(\rho, E)$ for all $x \geq x_{0}$. In $(\rho, E)$-plane, the trajectory of the solution is given by Equation (3.3). In this case, the trajectory is a loop with the center $(b, 0)$. The direction of the trajectory is counter clockwise. The solution is periodic and always supersonic.

Case 2: $\quad\left(\rho_{0}, E_{0}\right)$ is inside the critical subsonic branch of the critical trajectory, i.e., $\left(\frac{1}{2} E_{0}^{2}-\int_{\rho_{s}}^{\rho_{0}} H^{\prime}(s) d s<0\right.$ and $\left.\rho_{0}>\rho_{s}\right)$.

In this case, the initial value problem (3.1) admits a unique solution $(\rho, E)$ for all $x \geq x_{0} . E$ is strictly increasing. The solution is always subsonic. Moreover,

$$
\lim _{x \rightarrow \infty}(\rho, E)=(\infty, \infty) .
$$

Case 3: $\quad\left(\rho_{0}, E_{0}\right)$ is on the critical supersonic trajectory, i.e., $\frac{1}{2} E_{0}^{2}-\int_{\rho_{\mathrm{s}}}^{\rho_{0}} H^{\prime}(s) d s=0$ and $0<\rho_{0} \leq \rho_{s}$. In this case, there are infinitely many smooth solutions to the IVP (3.1) for all $x \geq x_{0}$. These solutions are of the following types:

i)(Type I) (Periodic) The solution $(\rho, E)$ is always on the supersonic loop of the critical trajectory.

ii) (Type II) The solution travels along the supersonic loop of the critical trajectory $n$ times $(n=0,1,2, \cdots)$, and then travels to the sonic point $\left(\rho_{s}, 0\right)$. From this sonic point, it travels along the upper subsonic branch of the critical trajectory $\frac{1}{2} E^{2}-\int_{\rho_{s}}^{\rho} H^{\prime}(s) d s=0, E>0, \rho>\rho_{s}$. In this case, we have

$$
\lim _{x \rightarrow \infty}(\rho, E)=(\infty, \infty) .
$$

Case 4: $\quad\left(\left(\rho_{0}, E_{0}\right)\right.$ is on the critical trajectory, and $\rho_{0}>\rho_{s}$ (subsonic) and $E_{0}>0$.)

In this case, there exists a unique solution $(\rho, E)(x)$ to the initial value problem (3.1) for all $x \geq x_{0}$, which travels along the upper subsonic branch of the critical trajectory $\frac{1}{2} E^{2}-\int_{\rho_{s}}^{\rho} H^{\prime}(s) d s=0, E>E_{0}, \rho>\rho_{0}$ satisfying

$$
\rho_{x}>0, E_{x}>0, \lim _{x \rightarrow \infty}(\rho, E)=(\infty, \infty) .
$$

Case 5: $\quad\left(\left(\rho_{0}, E_{0}\right)\right.$ is on the critical trajectory, and $\rho_{0}>\rho_{s}$ (subsonic) and $E_{0}<$ 0$.$) In this case, there are infinitely many solutions. In (\rho, E)$ plane, the solutions start from $\left(\rho_{0}, E_{0}\right)$, travel along the lower subsonic branch of the critical trajectory $\frac{1}{2} E^{2}-\int_{\rho_{s}}^{\rho} H^{\prime}(s) d s=0,0>E>E_{0}, \rho<\rho_{0}$ in the direction $\rho$ decreases and $E$ increases. The solutions reaches the sonic point $\left(\rho_{s}, 0\right)$ at some $x_{1}>x_{0}$. After then $\left(x>x_{1}\right)$, this case reduces to Case 3 .

Case 6: $\quad\left(\frac{1}{2} E_{0}^{2}-\int_{\rho_{s}}^{\rho_{0}} H^{\prime}(s) d s>0\right.$ and $\left.0<\rho_{0}<\rho_{s}.\right)$

In this case, the solution to the initial value problem (3.1) exists only on a finite interval $\left[x_{0}, x_{2}\right)$ for some $x_{2}>x_{0}$. Moreover,

$$
\lim _{x \rightarrow x_{2}^{-}}(\rho, E)=\left(\rho_{s}, E_{1}\right),
$$

where $E_{1}$ is determined by

$$
\frac{1}{2} E_{1}^{2}-\int_{\rho_{0}}^{\rho_{s}} H^{\prime}(s) d s=\frac{1}{2} E_{0}^{2}, E_{1}<0 .
$$


Furthermore,

$$
\lim _{x \rightarrow x_{2}^{-}} \rho_{x}(x)=+\infty
$$

Case 7: $\quad\left(\frac{1}{2} E_{0}^{2}-\int_{\rho_{s}}^{\rho_{0}} H^{\prime}(s) d s>0\right.$ and $\left.\rho_{0}>\rho_{s}, E_{0}>0.\right)$

In this case, the solution to the initial value problem (3.1) exists for all $x \geq x_{0}$. Along the trajectory of the solution, both $\rho$ and $E$ are strictly increasing. Moreover,

$$
\lim _{x \rightarrow \infty}(\rho, E)(x)=(+\infty,+\infty) .
$$

Case 8: $\quad\left(\frac{1}{2} E_{0}^{2}-\int_{\rho_{s}}^{\rho_{0}} H^{\prime}(s) d s>0\right.$ and $\left.\rho_{0}>\rho_{s}, E_{0}<0.\right)$

In this case, the solution to the initial value problem (3.1) exists only on a finite interval $\left[x_{0}, x_{3}\right)$ for some $x_{3}>x_{0}$. Moreover,

$$
\lim _{x \rightarrow x_{3}^{-}}(\rho, E)=\left(\rho_{s}, E_{2}\right)
$$

where $E_{2}$ is determined by

$$
\frac{1}{2} E_{2}^{2}-\int_{\rho_{0}}^{\rho_{s}} H^{\prime}(s) d s=\frac{1}{2} E_{0}^{2}, \quad E_{2}<0
$$

Furthermore,

$$
\lim _{x \rightarrow x_{3}^{-}} \rho_{x}(x)=-\infty
$$

3.2. The case when $b>\rho_{s}$. In the case when $b>\rho_{s}$, the equilibrium point $(b, 0)$ is a saddle point on the phase plane (see Figure 2).

One can also define the critical trajectory for the case $b>\rho_{s}$ as:

Definition 3.2. The critical trajectory (for the case $b>\rho_{s}$ ) is the trajectory passing through the point $(b, 0)$ with the equation:

$$
\frac{1}{2} E^{2}-\int_{b}^{\rho} H^{\prime}(s) d s=0 .
$$

The initial value problem 3.2 for the different cases of the initial data $\left(\rho_{0}, E_{0}\right)$ can be solved as follows.

Case $1\left(\rho_{0}<\rho_{s}\right)$, i.e., $\rho_{0}$ is supersonic. In this case, the solution of (3.1) only exists in a finite interval $\left[x_{0}, x_{4}\right)$. Moreover,

$$
\lim _{x \rightarrow x_{4}-}(\rho, E)=\left(\rho_{s},-\sqrt{E_{0}^{2}+2 \int_{\rho_{0}}^{\rho_{s}} H^{\prime}(s) d s}\right), \quad \lim _{x \rightarrow x_{4}-} \rho_{x}=+\infty .
$$

Case $2 \rho_{0}>\rho_{s}$.

a) $\left(\rho_{0}, E_{0}\right)$ is inside the critical trajectory, i.e.,

$$
\frac{1}{2} E_{0}^{2}+\int_{\rho_{0}}^{b} H^{\prime}(s) d s<0, \rho_{0}>\rho_{s} .
$$

There are two subcases. 
a1) $\rho_{s}<\rho_{0}<b$.

In this case, the initial value problem (3.1) admits a unique solution $(\rho, E)$ in a finite interval $\left[x_{0}, x_{5}\right)$. Moreover,

$$
\begin{aligned}
& b>\rho(x)>\rho_{s}, x \in\left[x_{0}, x_{5}\right), \\
& \lim _{x \rightarrow x_{5}-}(\rho, E)(x)=\left(\rho_{s},-\sqrt{E_{0}^{2}+2 \int_{\rho_{0}}^{\rho_{s}} H^{\prime}(s) d s}\right), \lim _{x \rightarrow x_{5}-} \rho_{x}(x)=-\infty .
\end{aligned}
$$

a2) $\rho_{0}>b$.

In this case, the initial value problem (3.1) admits a unique solution $(\rho, E)$ for all $x \geq x_{0}$. Moreover,

$$
\begin{aligned}
& \rho(x)>b>\rho_{s}, E_{x}>0, x \in\left[x_{0}, \infty\right), \\
& \lim _{x \rightarrow \infty}(\rho, E)(x)=(+\infty,+\infty) .
\end{aligned}
$$

b) $\left(\rho_{0}, E_{0}\right)$ is outside the critical trajectory, i.e.,

$$
\frac{1}{2} E_{0}^{2}+\int_{\rho_{0}}^{b} H^{\prime}(s) d s>0, \rho_{0}>\rho_{s} .
$$

There are two subcases.

b1) $E_{0}>0$.

In this case, the initial value problem (3.1) admits a unique solution $(\rho, E)$ for all $x \geq x_{0}$. Moreover,

$$
\begin{aligned}
& \rho(x)>\rho_{s}, x \in\left[x_{0}, \infty\right), \\
& \lim _{x \rightarrow \infty}(\rho, E)(x)=(+\infty,+\infty) .
\end{aligned}
$$

b2) $E_{0}<0$.

In this case, the initial value problem (3.1) admits a unique solution $(\rho, E)$ in a finite interval $\left[x_{0}, x_{6}\right)$. Moreover,

$$
\begin{aligned}
& \rho(x)>\rho_{s}, x \in\left[x_{0}, x_{6}\right), \\
& \lim _{x \rightarrow x_{6}-}(\rho, E)(x)=\left(\rho_{s},-\sqrt{E_{0}^{2}+2 \int_{\rho_{0}}^{\rho_{s}} H^{\prime}(s) d s}\right), \lim _{x \rightarrow x_{6}-} \rho_{x}(x)=-\infty .
\end{aligned}
$$

c) $\left(\rho_{0}, E_{0}\right)$ is on the critical supersonic trajectory, i.e.,

$$
\frac{1}{2} E_{0}^{2}+\int_{\rho_{0}}^{b} H^{\prime}(s) d s=0 .
$$

c1) $\rho_{s}<\rho_{0}<b, E_{0}>0$.

In this case, the initial value problem (3.1) admits a unique solution $(\rho, E)$ for all $x \geq x_{0}$. Moreover,

$$
\begin{gathered}
\rho_{x}>0, E_{x}<0, x>x_{0}, \\
\lim _{x \rightarrow \infty}(\rho, E)(x)=(b, 0) .
\end{gathered}
$$


c2) $\rho_{s}<\rho_{0}<b, E_{0}<0$.

In this case, the initial value problem (3.1) admits a unique solution $(\rho, E)$ in a finite interval $\left[x_{0}, x_{7}\right)$. Moreover,

$$
\begin{aligned}
& \rho_{x}(x)<0, E_{x}(x)<0, x \in\left[x_{0}, x_{7}\right), \\
& \lim _{x \rightarrow x_{7}-}(\rho, E)(x)=\left(\rho_{s},-\sqrt{2 \int_{b}^{\rho_{s}} H^{\prime}(s) d s}\right), \lim _{x \rightarrow x_{7}-} \rho_{x}(x)=-\infty .
\end{aligned}
$$

c3) $\rho_{0}>b, E_{0}>0$.

In this case, the initial value problem (3.1) admits a unique solution $(\rho, E)$ for all $x \geq x_{0}$. Moreover,

$$
\begin{aligned}
& \rho_{x}>0, E_{x}>0, x>x_{0}, \\
& \lim _{x \rightarrow \infty}(\rho, E)(x)=(\infty, \infty) .
\end{aligned}
$$

c4) $\rho_{0}>b, E_{0}<0$.

In this case, the initial value problem (3.1) admits a unique solution $(\rho, E)$ for all $x \geq x_{0}$. Moreover,

$$
\begin{aligned}
& \rho_{x}(x)>0, E_{x}(x)>0, x>x_{0}, \\
& \lim _{x \rightarrow \infty}(\rho, E)(x)=(b, 0) .
\end{aligned}
$$

\section{Some general lemmas}

In this section, we give several lemmas which will be used later. In what follows, $(\rho, E)\left(x, \rho_{0}, E_{0}\right)\left(x \geq x_{0}\right)$ denotes the solution of the initial value problem (3.1) and $T\left(\rho_{0}, E_{0}\right)$ presents the trajectory passing through the state $\left(\rho_{0}, E_{0}\right)$ in the direction as $x$ increases. Precisely, we define

Definition 4.1. We say that a state $\left(\rho_{1}, E_{1}\right) \in T\left(\rho_{0}, E_{0}\right)$ if there exist $x_{0} \in \mathrm{R}^{1}$ and $x_{1} \in \mathrm{R}^{1}$ satisfying $x_{1} \geq x_{0}$ such that $\left(\rho_{1}, E_{1}\right)=(\rho, E)\left(x_{1}, \rho_{0}, E_{0}\right)$.

Therefore, if $\left(\rho_{1}, E_{1}\right) \in T\left(\rho_{0}, E_{0}\right)$, then

$$
\frac{1}{2} E_{1}^{2}-\int_{\rho_{0}}^{\rho_{1}} H^{\prime}(s) d s=\frac{1}{2} E_{0}^{2}
$$

For any $\rho \in\left(0, \rho_{s}\right)$, there exists one and only one $F(\rho)$ satisfying

$$
p(F(\rho))+\frac{J^{2}}{F(\rho)}=p(\rho)+\frac{J^{2}}{\rho}, \quad F(\rho)>\rho_{s},
$$

i.e., the state $(\rho, E)$ and $(F(\rho), E)$ can be connected by a transonic shock (cf. the Rankine-Hogoniout condition 2.1). It is easy to verify that

$$
\begin{gathered}
F^{\prime}(\rho)=\frac{p^{\prime}(\rho)-\frac{J^{2}}{\rho^{2}}}{p^{\prime}(F(\rho))-\frac{J^{2}}{F(\rho)^{2}}}<0, \text { for } 0<\rho<\rho_{s}, \\
H^{\prime}(F(\rho)) F^{\prime}(\rho)=\frac{F(\rho)-b}{F(\rho)}\left(p^{\prime}(\rho)-\frac{J^{2}}{\rho^{2}}\right), \text { for } 0<\rho<\rho_{s} .
\end{gathered}
$$


For the trajectory passing through $\left(\rho_{l}, \alpha\right)$, the shock curve is defined by $T_{\text {shock}}$ :

$$
T_{\text {shock }}=\left\{(F(\rho), E):(\rho, E) \in T\left(\rho_{l}, \alpha\right)\right\} .
$$

Let $\ell\left(\left(\rho_{1}, E_{1}\right) ;\left(\rho_{2}, E_{2}\right)\right)$ be the length in $x$ for the trajectory of (1.7) to travel from the state $\left(\rho_{1}, E_{1}\right)$ to the state $\left(\rho_{2}, E_{2}\right)$ when $\left(\rho_{1}, E_{1}\right)$ and $\left(\rho_{2}, E_{2}\right)$ are on the same trajectory. When $\left(\rho_{1}, E_{1}\right)$ and $\left(\rho_{2}, E_{2}\right)$ are on the same periodic trajectory, $\ell\left(\left(\rho_{1}, E_{1}\right) ;\left(\rho_{2}, E_{2}\right)\right)$ is understood as the length in $x$ which does not exceed one period. Some elementary but important properties of these trajectories and shock wave curves are listed in the following lemmas.

Lemma 4.2. Suppose that the two states $\left(\rho_{1}, E_{1}\right)$ and $\left(\rho_{2}, E_{2}\right)$ are on the same trajectory of system (1.7), i.e., $\left(\rho_{2}, E_{2}\right) \in T\left(\rho_{1}, E_{1}\right)$. Then

i) if on the trajectory connecting these two states, $E$ does not change sign (then $E$ is a function of $\rho$, denoted by $E(\rho)$ ),

$$
\ell\left(\left(\rho_{1}, E_{1}\right) ;\left(\rho_{2}, E_{2}\right)\right)=\int_{\rho_{1}}^{\rho_{2}} \frac{p^{\prime}(\rho)-\frac{J^{2}}{\rho^{2}}}{\rho E(\rho)} d \rho
$$

ii) if on the trajectory connecting these two states, $E$ is strictly increasing or decreasing (then $\rho$ is a function of $E$, denoted by $\rho\left(E, \rho_{1}\right)$ ), then

$$
\ell\left(\left(\rho_{1}, E_{1}\right) ;\left(\rho_{2}, E_{2}\right)\right)=\int_{E_{1}}^{E_{2}} \frac{d E}{\rho\left(E, \rho_{1}\right)-b},
$$

as long as $\rho\left(E, \rho_{1}\right) \neq b$ for $E$ between $E_{1}$ and $E_{2}$.

Proof. It follows from $(1.7)_{1}$ that $\frac{p^{\prime}(\rho)-\frac{J^{2}}{\rho^{2}}}{\rho E} d \rho=d x$ when $E$ does not change sign. This proves (4.4), while the second equation in (1.7) yields $\frac{d E}{\rho-b}=d x$. Thus (4.5) follows.

Lemma 4.3. For fixed $\left(\rho_{0}, E_{0}\right)$ and $\rho_{r}$, let

$$
X(\bar{\rho})=\ell\left(\left(\rho_{0}, E_{0}\right) ;(\bar{\rho}, E(\bar{\rho}))\right)+\ell\left((F(\bar{\rho}), E(\bar{\rho})) ;\left(\rho_{r}, E_{r}(\bar{\rho})\right)\right),
$$

where $\rho_{0}<\rho_{s}, \bar{\rho}<\rho_{s}, \rho_{r}>\rho_{s}, \quad(\bar{\rho}, E(\bar{\rho})) \in T\left(\rho_{0}, E_{0}\right), \quad\left(\rho_{r}, E_{r}(\bar{\rho})\right) \in T(F(\bar{\rho}), E(\bar{\rho}))$. If $E$ does not change sign along the trajectories from $\left(\rho_{0}, E_{0}\right)$ to $(\bar{\rho}, E(\bar{\rho}))$ and from $(F(\bar{\rho}), E(\bar{\rho}))$ to $\left(\rho_{r}, E_{r}(\bar{\rho})\right)$, then

$$
X^{\prime}(\bar{\rho})=\left(p^{\prime}(\bar{\rho})-\frac{J^{2}}{\bar{\rho}^{2}}\right)\left(\frac{1}{\bar{\rho}}-\frac{1}{F(\bar{\rho})}\right) Q(\bar{\rho}),
$$

provided $E(\bar{\rho}) \neq 0$, where

$$
Q(\bar{\rho})=\frac{1}{E(\bar{\rho})}+b \int_{F(\bar{\rho})}^{\rho_{r}} \frac{p^{\prime}(t)-\frac{J^{2}}{t^{2}}}{t E^{3}(\bar{\rho}, t)} d t
$$

and

$$
E(\bar{\rho}, t)=\operatorname{sgn}(E(\bar{\rho})) \sqrt{E^{2}(\bar{\rho})+2 \int_{F(\bar{\rho})}^{t} H^{\prime}(s) d s},
$$


for $t$ between $F(\bar{\rho})$ and $\rho_{r}$. Moreover,

$$
Q^{\prime}(\bar{\rho})=\left(p^{\prime}(\bar{\rho})-\frac{J^{2}}{\bar{\rho}^{2}}\right)\left(\frac{1}{E^{3}(\bar{\rho})}\left[\frac{b}{\bar{\rho}}-\frac{b}{F(\bar{\rho})}-1\right]+3 b^{2}\left[\frac{1}{\bar{\rho}}-\frac{1}{F(\bar{\rho})}\right] \int_{F(\bar{\rho})}^{\rho_{r}} \frac{p^{\prime}(t)-\frac{J^{2}}{t^{2}}}{t E^{5}(\bar{\rho}, t)} d t\right) .
$$

Proof. Let $X_{1}(\bar{\rho})=\ell\left(\left(\rho_{0}, E_{0}\right) ;(\bar{\rho}, E(\bar{\rho}))\right)$ and $X_{2}(\bar{\rho})=\ell\left((F(\bar{\rho}), E(\bar{\rho})) ;\left(\rho_{r}, E_{r}(\bar{\rho})\right)\right)$. Then it follows from Lemma 4.2 that

$$
X_{1}(\bar{\rho})=\int_{\rho_{0}}^{\bar{\rho}} \frac{p^{\prime}(t)-\frac{J^{2}}{t^{2}}}{t E(t)} d t, X_{2}(\bar{\rho})=\int_{F(\bar{\rho})}^{\rho_{r}} \frac{p^{\prime}(t)-\frac{J^{2}}{t^{2}}}{t E(\bar{\rho}, t)} d t
$$

where $(t, E(t)) \in T\left(\rho_{0}, E_{0}\right)$ for $t$ between $\rho_{0}$ and $\bar{\rho}$, and $E(\bar{\rho}, t)$ is given by

$$
\frac{1}{2} E^{2}(\bar{\rho}, t)-H(t)=\frac{1}{2} E^{2}(\bar{\rho})-H(F(\bar{\rho})),
$$

for $t$ between $F(\bar{\rho})$ and $\rho_{r}$. Since $(\bar{\rho}, E(\bar{\rho})) \in T\left(\rho_{0}, E_{0}\right)$, it holds that

$$
\frac{1}{2} E^{2}(\bar{\rho})-H(\bar{\rho})=\frac{1}{2} E_{0}^{2}-H\left(\rho_{0}\right) .
$$

Therefore

$$
E(\bar{\rho}) E^{\prime}(\bar{\rho})=H^{\prime}(\bar{\rho})
$$

By (4.11), one can get

$$
X_{1}^{\prime}(\bar{\rho})=\frac{p^{\prime}(\bar{\rho})-\frac{J^{2}}{\bar{\rho}^{2}}}{\bar{\rho} E(\bar{\rho})} .
$$

Noting that $E(\bar{\rho}, t)=E(\bar{\rho})$ as $t=F(\bar{\rho})$ in (4.12), and using the second equation in (4.11), we obtain

$$
\begin{aligned}
X_{2}^{\prime}(\bar{\rho})= & -\frac{p^{\prime}(F(\bar{\rho}))-\frac{J^{2}}{(F(\bar{\rho}))^{2}}}{F(\bar{\rho}) E(\bar{\rho})} F^{\prime}(\bar{\rho}) \\
& -\int_{F(\bar{\rho})}^{\rho_{r}} \frac{\left(p^{\prime}(t)-\frac{J^{2}}{t^{2}}\right) \partial E(\bar{\rho}, t) / \partial \bar{\rho}}{t E^{2}(\bar{\rho}, t)} d t .
\end{aligned}
$$

By virtue of (4.3), (4.12), and (4.13), one has

$$
\begin{aligned}
E(\bar{\rho}, t) \frac{\partial E(\bar{\rho}, t)}{\partial \bar{\rho}} & =E(\bar{\rho}) E^{\prime}(\bar{\rho})-H^{\prime}\left(F(\bar{\rho}) F^{\prime}(\bar{\rho})\right. \\
& =H^{\prime}(\bar{\rho})-H^{\prime}(F(\bar{\rho})) F^{\prime}(\bar{\rho}) \\
& =\left(p^{\prime}(\bar{\rho})-\frac{J^{2}}{\bar{\rho}^{2}}\right)\left(\frac{b}{F(\bar{\rho})}-\frac{b}{\bar{\rho}}\right) .
\end{aligned}
$$

Since $X(\bar{\rho})=X_{1}(\bar{\rho})+X_{2}(\bar{\rho})$, (4.7) follows from (4.15)-(4.17). (4.10) can be obtained by the same method.

The following alternative lemma will be used later. 
LEMMA 4.4. For the fixed $\left(\rho_{0}, E_{0}\right)$ and $\rho_{r}$ satisfying $\rho_{0}<\rho_{s}, \rho_{r}>\rho_{s}$, assume that $(\bar{\rho}, E(\bar{\rho})) \in T\left(\rho_{0}, E_{0}\right)$ satisfies $0<\bar{\rho}<\rho_{s}$ and $E$ does not change sign along the trajectory from $\left(\rho_{0}, E_{0}\right)$ to $(\bar{\rho}, E(\bar{\rho}))$. Moreover, suppose that the trajectory starting from $(F(\bar{\rho}), E(\bar{\rho}))$ crosses the $\rho$-axis at the point $(q(\bar{\rho}), 0)$ and then intersects the line $\rho=\rho_{r}$ at $\left(\rho_{r}, E_{r}(\bar{\rho})\right)$ (i.e. $(q(\bar{\rho}), 0) \in T(F(\bar{\rho}), E(\bar{\rho}))$ and $\left.\left(\rho_{r}, E_{r}(\bar{\rho})\right) \in T(q(\bar{\rho}), 0)\right)$. Assume further that $\rho \neq b$ on the trajectory $T(F(\bar{\rho}), E(\bar{\rho}))$. Let

$$
X(\bar{\rho})=\ell\left(\left(\rho_{0}, E_{0}\right) ;(\bar{\rho}, E(\bar{\rho}))\right)+\ell\left((F(\bar{\rho}), E(\bar{\rho})) ;\left(\rho_{r}, E_{r}(\bar{\rho})\right)\right) .
$$

Then

$$
\begin{gathered}
\frac{d X(\bar{\rho})}{d \bar{\rho}}=\left(p^{\prime}(\bar{\rho})\right. \\
\left.-\frac{J^{2}}{\bar{\rho}^{2}}\right) \frac{F(\bar{\rho})-\bar{\rho}}{\bar{\rho}}\left\{\frac{1}{(F(\bar{\rho})-b) E(\bar{\rho})}-\frac{b}{F(\bar{\rho})}\left[\frac{1}{\left(\rho_{r}-b\right) E_{r}(\bar{\rho})}\right.\right. \\
\left.\left.+\int_{F(\bar{\rho})}^{q(\bar{\rho})} \frac{d \rho}{(\rho-b)^{2} E_{1}(\rho, \bar{\rho})}+\int_{q(\bar{\rho})}^{\rho_{r}} \frac{d \rho}{(\rho-b)^{2} E_{2}(\rho, \bar{\rho})}\right]\right\},
\end{gathered}
$$

provided $E(\bar{\rho}) \neq 0$ and $E_{r}(\bar{\rho}) \neq 0$, where

$$
\begin{gathered}
E_{1}(\rho, \bar{\rho})=\operatorname{sgn}(E(\bar{\rho})) \sqrt{E^{2}(\bar{\rho})+2 \int_{F(\bar{\rho})}^{\rho} H^{\prime}(t) d t} \\
E_{2}(\rho, \bar{\rho})=-\operatorname{sgn}(E(\bar{\rho})) \sqrt{E^{2}(\bar{\rho})+2 \int_{F(\bar{\rho})}^{\rho} H^{\prime}(t) d t} .
\end{gathered}
$$

Proof. By the definition of $E_{1}(\rho, \bar{\rho}), E_{2}(\rho, \bar{\rho})$, and $q(\bar{\rho})$, it is clear that

$$
\begin{gathered}
E_{1}(q(\bar{\rho}), \bar{\rho})=E_{2}(q(\bar{\rho}), \bar{\rho})=0, \\
E_{1}(F(\bar{\rho}), \bar{\rho})=E(\bar{\rho}), E_{2}\left(\rho_{r}, \bar{\rho}\right)=E_{r}(\bar{\rho}) .
\end{gathered}
$$

Let

$$
Y_{1}(\bar{\rho})=\ell\left(\left(\rho_{0}, E_{0}\right) ;(\bar{\rho}, E(\bar{\rho}))\right)
$$

and

$$
Y_{2}(\bar{\rho})=\ell\left((F(\bar{\rho}), E(\bar{\rho})) ;\left(\rho_{r}, E_{r}(\bar{\rho})\right)\right) .
$$

Similar to (4.14), one has

$$
Y_{1}^{\prime}(\bar{\rho})=\frac{p^{\prime}(\bar{\rho})-\frac{J^{2}}{\bar{\rho}^{2}}}{\bar{\rho} E(\bar{\rho})}
$$

as long as $E(\bar{\rho}) \neq 0$. The Equation $\frac{d E_{1}(\rho, \bar{\rho})}{d x}=\rho-b$ yields that

$$
L_{2}(\bar{\rho}):=\ell((F(\bar{\rho}), E(\bar{\rho})) ;(q(\bar{\rho}), 0))=\int_{F(\bar{\rho})}^{q(\bar{\rho})} \frac{\partial E_{1}(\rho, \bar{\rho}) / \partial \rho}{\rho-b} d \rho .
$$

Due to (4.21) and (4.22), integration by parts gives

$$
L_{2}(\bar{\rho})=-\frac{E(\bar{\rho})}{F(\bar{\rho})-b}+\int_{F(\bar{\rho})}^{q(\bar{\rho})} \frac{E_{1}(\rho, \bar{\rho})}{(\rho-b)^{2}} d \rho .
$$


Similarly,

$$
L_{3}(\bar{\rho}):=\ell\left((q(\bar{\rho}), 0) ;\left(\rho_{r}, E_{r}(\bar{\rho})\right)\right)=\frac{E_{r}(\bar{\rho})}{\rho_{r}-b}+\int_{q(\bar{\rho})}^{\rho_{r}} \frac{E_{2}(\rho, \bar{\rho})}{(\rho-b)^{2}} d \rho .
$$

It should be noted that

$$
\ell\left((F(\bar{\rho}), E(\bar{\rho})) ;\left(\rho_{r}, E_{r}(\bar{\rho})\right)\right)=L_{2}(\bar{\rho})+L_{3}(\bar{\rho}) .
$$

By (4.26), (4.21), and (4.22), we have,

$$
L_{2}^{\prime}(\bar{\rho})=-\frac{E^{\prime}(\bar{\rho})}{F(\bar{\rho})-b}+\int_{F(\bar{\rho})}^{q(\bar{\rho})} \frac{\partial E_{1}(\rho, \bar{\rho}) / \bar{\rho}}{(\rho-b)^{2}} d \rho
$$

An argument similar to that for (4.14) shows

$$
E^{\prime}(\bar{\rho}) E^{\prime}(\bar{\rho})=H^{\prime}(\bar{\rho}) .
$$

It follows from (4.19) that

$$
\frac{1}{2} E_{1}^{2}(\rho, \bar{\rho})=\frac{1}{2} E^{2}(\bar{\rho})+\int_{F(\bar{\rho})}^{\rho} H^{\prime}(t) d t
$$

Therefore, in view of (4.30), one obtains that

$$
E_{1}(\rho, \bar{\rho}) \frac{\partial E_{1}}{\partial \bar{\rho}}=H^{\prime}(\bar{\rho})-H^{\prime}(F(\bar{\rho})) F^{\prime}(\bar{\rho})
$$

This then yields, with the help of (4.30), (4.31), and (4.32), that

$$
L_{2}^{\prime}(\bar{\rho})=-\frac{H^{\prime}(\bar{\rho})}{(F(\bar{\rho})-b) E(\bar{\rho})}+\left(H^{\prime}(\bar{\rho})-H^{\prime}(F(\bar{\rho})) F^{\prime}(\bar{\rho})\right) \int_{F(\bar{\rho})}^{q(\bar{\rho})} \frac{1}{(\rho-b)^{2} E_{1}(\rho, \bar{\rho})} d \rho .
$$

Now we show that $\int_{F(\bar{\rho})}^{q(\bar{\rho})} \frac{1}{(\rho-b)^{2} E_{1}(\rho, \bar{\rho})} d \rho$ is finite. This is necessary because $\left.E_{1}(q(\bar{\rho}), \bar{\rho})\right)=0$. Let

$$
g(\rho)=E^{2}(\bar{\rho})+2 \int_{F(\bar{\rho})}^{\rho} H^{\prime}(t) d t
$$

Then (4.19) yields that

$$
E_{1}(\rho, \bar{\rho})=\operatorname{sgn}(E(\bar{\rho})) \sqrt{g(\rho)}
$$

Clearly, $g(q(\bar{\rho}))=0$ and

$$
g^{\prime}(q(\bar{\rho}))=H^{\prime}(q(\bar{\rho})) \neq 0
$$

because $q(\bar{\rho}) \neq b$ and $q(\bar{\rho}) \neq \rho_{s}$. So

$$
g(\rho)=g^{\prime}(q(\bar{\rho}))(\rho-q(\bar{\rho}))+O\left((\rho-q(\bar{\rho}))^{2}\right),
$$

as $|\rho-q(\bar{\rho})|$ is small. This, together with (4.33) and (4.34), implies that $\int_{F(\bar{\rho})}^{q(\bar{\rho})} \frac{1}{(\rho-b)^{2} E_{1}(\rho, \bar{\rho})} d \rho$ is finite. Similarly, one can show that

$$
L_{3}^{\prime}(\bar{\rho})=\left(H^{\prime}(\bar{\rho})-H^{\prime}(F(\bar{\rho})) F^{\prime}(\bar{\rho})\right)\left(\frac{1}{\left(\rho_{r}-b\right) E_{r}(\bar{\rho})}+\int_{q(\bar{\rho})}^{\rho_{r}} \frac{1}{(\rho-b)^{2} E_{2}(\rho, \bar{\rho})} d \rho\right) .
$$

Then (4.18) follows from (4.24), (4.32), and (4.35), in view of (4.2).

In the following, since the behavior of solutions for $0<b<\rho_{s}$ and $b>\rho_{s}$ are completely different, we construct transonic shock solutions for those two cases separately. 
5. Transonic shock solutions for the case of $0<b<\rho_{s}$

We consider this problem according to the relative position of $\left(\rho_{1}, \alpha\right)$.

The first case is that $\left(\rho_{l}, \alpha\right)$ is inside the critical trajectory. In this case, by Case 1 discussed in Section 2, the initial value problem

$$
\left\{\begin{array}{l}
\left(p(\rho)+\frac{J^{2}}{\rho}\right)_{x}=\rho E, \quad E_{x}=\rho-b, \text { for } x>0, \\
(\rho, E)(0)=\left(\rho_{l}, \alpha\right)
\end{array}\right.
$$

has a unique periodic supersonic solution. Denote the period of the solution bey $P$. Let $\rho_{L}(x)$ be the solution of initial value problem (5.1), and

$$
\rho_{\min }=: \min _{0 \leq x \leq P} \rho_{L}(x), \rho_{\max }=: \max _{0 \leq x \leq P} \rho_{L}(x) .
$$

Then $\rho_{\min }$ and $\rho_{\max }$ are two solutions of the following equation for $\rho$ :

$$
\frac{1}{2} \alpha^{2}=\int_{\rho}^{\rho_{l}} H^{\prime}(s) d s
$$

So $\left(\rho_{\min }, 0\right)$ and $\left(\rho_{\max }, 0\right)$ are the two intersection points of the trajectory for $(5.1)$ with the line $E=0$ on the $(\rho, E)$-plane (see Figure 3 and Figure 4$)$. Then we have the following theorem:

Theorem 5.1. Suppose that $0<b<\rho_{s}$ and $\left(\rho_{l}, \alpha\right)$ is inside the supersonic loop of critical trajectory, i.e.,

$$
\frac{1}{2} \alpha^{2}-\int_{\rho_{s}}^{\rho_{l}} H^{\prime}(s) d s<0, \text { and } 0<\rho_{l}<\rho_{s}
$$

and

$$
\left(\rho_{l}, \alpha\right) \neq(b, 0)
$$

and assume that

$$
\rho_{r}>F\left(\rho_{\min }\right)
$$

Then

1) If

$$
L<\ell\left(\left(F\left(\rho_{l}\right), \alpha\right) ;\left(\rho_{r}, E_{\max }\right)\right),
$$

the boundary value problem (1.7) and (1.8) does not have a transonic shock solution; 2) If

$$
L \geq \ell\left(\left(F\left(\rho_{l}\right), \alpha\right) ;\left(\rho_{r}, E_{\max }\right)\right),
$$

then the boundary value problem (1.7) and (1.8) admits a unique transonic shock solution containing a single transonic shock, the location of the transonic shock is uniquely determined by the boundary data $\left(\rho_{l}, \alpha\right), \rho_{r}$, and the interval length $L$.

Proof. For $L \geq \ell\left(\left(F\left(\rho_{l}\right), \alpha\right) ;\left(\rho_{r}, E_{\max }\right)\right)$, there exists an integer $k \geq 0$ such that

$$
k P+\ell\left(\left(F\left(\rho_{l}\right), \alpha\right) ;\left(\rho_{r}, E_{\max }\right)\right) \leq L<(k+1) P+\ell\left(\left(F\left(\rho_{l}\right), \alpha\right) ;\left(\rho_{r}, E_{\max }\right)\right) .
$$

We shall prove that the length $\ell\left(\left(\rho_{l}, \alpha\right) ;(\rho, E)\right)+\ell\left((F(\rho), E) ;\left(\rho_{r}, E_{\max }\right)\right)$ is strictly increasing as $(\rho, E) \in T\left(\rho_{l}, \alpha\right)$ and moves counterclockwise along the trajectory of (5.1) starting from $\left(\rho_{l}, \alpha\right)$. Then 1) and 2) follow immediately. We will treat 
only the case that $\alpha>0$, since the case when $\alpha \leq 0$ can be handled similarly. The proof consists of several steps.

Step 1. For any $\bar{\rho} \in\left[\rho_{\text {min }}, \rho_{l}\right]$, let

$$
\begin{gathered}
E(\bar{\rho})=\sqrt{\alpha^{2}+2 \int_{\rho_{l}}^{\bar{\rho}} H^{\prime}(s) d s,} \\
E_{r}(\bar{\rho})=\sqrt{E^{2}(\bar{\rho})+2 \int_{F(\bar{\rho})}^{\rho_{r}} H^{\prime}(s) d s},
\end{gathered}
$$

i.e., $\left(\rho_{l}, \alpha\right)$ and $(\bar{\rho}, E(\bar{\rho}))$ are on the same supersonic trajectory of $(1.7)$ and $\left(\rho_{r}, E_{r}(\bar{\rho})\right)$ and $(F(\bar{\rho}), E(\bar{\rho}))$ are on the same subsonic trajectory. Let

$$
X_{1}(\bar{\rho})=\ell\left(\left(\rho_{l}, \alpha\right) ;(\bar{\rho}, E(\bar{\rho}))\right)+\ell\left((F(\bar{\rho}), E(\bar{\rho})) ;\left(\rho_{r}, E_{r}(\bar{\rho})\right)\right) \text {, for } \rho_{\min }<\rho \leq \rho_{l} .
$$

Then Lemma 4.3 yields

$$
X_{1}^{\prime}(\bar{\rho})<0, \text { for } \rho_{\min }<\rho \leq \rho_{l},
$$

where one has used the facts that $0<\bar{\rho}<\rho_{s}<F(\bar{\rho})<\rho_{r}, E(\bar{\rho})>0$, and $0<b<\rho_{s}$.

Step 2. For $\bar{\rho} \in\left[\rho_{\min }, \rho_{\max }\right]$, we define

$$
\begin{gathered}
E(\bar{\rho})=-\sqrt{2 \int_{\rho_{\text {min }}}^{\bar{\rho}} H^{\prime}(s) d s .} \\
E_{r}(\bar{\rho})=\sqrt{E(\bar{\rho})^{2}+2 \int_{F(\bar{\rho})}^{\rho_{r}} H^{\prime}(s) d s},
\end{gathered}
$$

i.e., $\left(\rho_{\min }, 0\right)$ and $(\bar{\rho}, E(\bar{\rho}))$ are on the same supersonic trajectory and $\left(\rho_{r}, E_{r}(\bar{\rho})\right)$ and $(F(\bar{\rho}), E(\bar{\rho}))$ are on the same subsonic trajectory. Let

$$
X_{2}(\bar{\rho})=\ell\left(\left(\rho_{\min }, 0\right) ;(\bar{\rho}, E(\bar{\rho}))\right)+\ell\left((F(\bar{\rho}), E(\bar{\rho})) ;\left(\rho_{r}, E_{r}(\bar{\rho})\right)\right), \quad \text { for } \quad \rho_{\min }<\bar{\rho} \leq \rho_{\max } .
$$

Then one can apply Lemma 4.3 again to show that

$$
X_{2}^{\prime}(\bar{\rho})>0, \text { for } \bar{\rho}_{\min }<\rho<\rho_{\max }
$$

due to the facts that $0<b<\rho_{s}$, and for $\rho_{\min }<\bar{\rho}<\rho_{\max }, 0<\bar{\rho}<\rho_{s}, E(\bar{\rho})<0, E_{r}(\bar{\rho})>$ $0, \rho_{r}>\rho_{s}>b, q(\bar{\rho})<F(\bar{\rho})$ and $q(\bar{\rho})<F(\bar{\rho})$. Moreover,

$$
E_{1}(\rho, \bar{\rho})<0, \quad \text { for } q(\bar{\rho})<\rho \leq F(\bar{\rho}),
$$

and

$$
E_{1}(\rho, \bar{\rho})>0 \text {, for } q(\bar{\rho})<\rho \leq \rho_{r} .
$$

These quantities are defined in Lemma 4.3.

Step 3. For $\bar{\rho} \in\left[\rho_{l}, \rho_{\max }\right]$, we define

$$
E(\bar{\rho})=\sqrt{2 \int_{\rho_{\max }}^{\bar{\rho}} H^{\prime}(s) d s},
$$




$$
E_{r}(\rho)=\sqrt{E(\bar{\rho})^{2}+2 \int_{F(\bar{\rho})}^{\rho_{r}} H^{\prime}(s) d s}
$$

i.e., $\left(\rho_{\max }, 0\right)$ and $(\bar{\rho}, E(\bar{\rho}))$ are on the same supersonic trajectory of (1.7) and $\left(\rho_{r}, E_{r}(\bar{\rho})\right)$ and $(F(\bar{\rho}), E(\bar{\rho}))$ are on the same subsonic trajectory. Let

$$
X_{3}(\bar{\rho})=\ell\left(\left(\rho_{\max }, 0\right) ;(\bar{\rho}, E(\bar{\rho}))\right)+\ell\left((F(\bar{\rho}), E(\bar{\rho})) ;\left(\rho_{r}, E_{r}(\bar{\rho})\right)\right) \text {, for } \rho_{l}<\bar{\rho}<\rho_{\max } .
$$

A similar analysis as in the proof of Step 1 shows that

$$
X_{3}^{\prime}(\bar{\rho})<0, \text { for } \rho_{l}<\rho<\rho_{\max } .
$$

Based on these monotonicity properties, the transonic solutions can be shown to have the structure as described in the following. We only describe the case for $k=0$, since for other values of $k$, starting from the state $\left(\rho_{l}, \alpha\right)$, the solution first travels $k$ times along the periodic trajectory of (5.1) and comes back to the state $\left(\rho_{l}, \alpha\right)$ at $x=k P$.

(i) When

$$
\ell\left(\left(F\left(\rho_{l}\right), \alpha\right) ;\left(\rho_{r}, E_{\max }\right)\right) \leq L \leq \ell\left(\left(\rho_{l}, \alpha\right),\left(\rho_{\min }, 0\right)\right)+\ell\left(\left(F\left(\rho_{\min }\right), 0\right) ;\left(\rho_{r}, E_{\text {min }}\right)\right),
$$

with $E_{\max }=\sqrt{\alpha^{2}+2 \int_{F\left(\rho_{l}\right)}^{\rho_{r}} H^{\prime}(\rho) d \rho}$ and $E_{\min }=\sqrt{\alpha^{2}+2 \int_{F\left(\rho_{\min }\right)}^{\rho_{r}} H^{\prime}(\rho) d \rho}$, there exist a unique state $\left(\rho^{*}, E^{*}\right)$ on the trajectory of system (1.7) passing through $\left(\rho_{l}, \alpha\right)$ satisfying $\rho_{\min } \leq \rho^{*} \leq \rho_{l}$ and $E^{*} \geq 0$ and a unique number $\beta$ satisfying $E_{\min } \leq \beta \leq E_{\max }$ such that the following equality holds true:

$$
L=\ell\left(\left(\rho_{l}, \alpha\right) ;\left(\rho^{*}, E^{*}\right)\right)+\ell\left(\left(F\left(\rho^{*}\right), E^{*}\right) ;\left(\rho_{r}, \beta\right)\right) .
$$

Thus the transonic shock location is $a=\ell\left(\left(\rho_{l}, \alpha\right) ;\left(\rho^{*}, E^{*}\right)\right)$.

(ii) If $L_{3}+L_{4}<L<L_{3}+L_{5}+L_{6}$, where

$$
L_{3}=\ell\left(\left(\rho_{l}, \alpha\right) ;\left(\rho_{\min }, 0\right)\right), L_{4}=\ell\left(\left(F\left(\rho_{\min }\right), 0\right) ;\left(\rho_{r}, E_{\min }\right)\right),
$$

with $E_{\text {min }}=\sqrt{2 \int_{F\left(\rho_{\min }\right)}^{\rho_{r}} H^{\prime}(s) d s}$,

$$
L_{5}=\ell\left(\left(\rho_{\min }, 0\right) ;\left(\rho_{\max }, 0\right)\right), L_{6}=\ell\left(\left(F\left(\rho_{\max }\right), 0\right) ;\left(\rho_{r}, \tilde{E}\right)\right),
$$

with $\tilde{E}=\sqrt{2 \int_{F\left(\rho_{\max }\right)}^{\rho_{r}} H^{\prime}(s) d s}$ and $E_{\max }=\sqrt{\alpha^{2}+2 \int_{F\left(\rho_{l}\right)}^{\rho_{r}} H^{\prime}(s) d s}$, there exist a unique number $\beta_{1}$ and a unique state $\left(\rho_{*}, E_{*}\right)$ on the trajectory of system (1.7) passing through $\left(\rho_{l}, \alpha\right)$ satisfying $\rho_{\min }<\rho_{*}<\rho_{\max }$ and $E_{*}<0$ such that the following equality holds true:

$$
L=\ell\left(\left(\rho_{l}, \alpha\right) ;\left(\rho_{*}, E_{*}\right)\right)+\ell\left(\left(F\left(\rho_{*}\right), E_{*}\right) ;\left(\rho_{r}, \beta_{1}\right)\right) .
$$

So the transonic shock location is $a=\ell\left(\left(\rho_{l}, \alpha\right) ;\left(\rho_{*}, E_{*}\right)\right)$.

(iii) If

$$
L_{3}+L_{5}+L_{6}<L<L_{3}+L_{5}+L_{7}+L_{8}
$$


where $L_{i}(i=3,5,6)$ are defined in (ii) above, and

$$
L_{7}=\ell\left(\left(\rho_{\max }, 0\right) ;\left(\rho_{l}, \alpha\right)\right), L_{8}=\ell\left(F\left(\rho_{l}, \alpha\right) ;\left(\rho_{r}, E_{\alpha}\right)\right),
$$

with $E_{\alpha}=\sqrt{\alpha^{2}+2 \int_{F\left(\rho_{l}\right)}^{\rho^{r}} H^{\prime}(s) d s}$, there exist a unique number $\beta_{2}$ and a unique state $\left(\rho^{* *}, E^{* *}\right)$ on the trajectory of $(1.7)$ passing through $\left(\rho_{l}, \alpha\right)$ satisfying $\rho_{l}<\rho^{* *}<\rho_{\max }$ and $E^{* *}>0$ such that the following equality holds true:

$$
L=\ell\left(\left(\rho_{l}, \alpha\right) ;\left(\rho^{* *}, E^{* *}\right)\right)+\ell\left(\left(F\left(\rho^{* *}\right), E * *\right) ;\left(\rho_{r}, \beta_{2}\right)\right) .
$$

Hence the transonic shock location is $\ell\left(\left(\rho_{l}, \alpha\right) ;\left(\rho^{* *}, E^{* *}\right)\right)$.

We now turn to the case when $\left(\rho_{l}, \alpha\right)$ is on the supersonic loop of the critical trajectory, i.e.,

$$
\frac{1}{2} \alpha^{2}-\int_{\rho_{s}}^{\rho_{l}} H^{\prime}(s) d s=0, \rho_{l}<\rho_{s}
$$

There are two intersection points of the supersonic loop of the critical trajectory and the line $E=0$. One is $\left(\rho_{s}, 0\right)$, another one is $\left(\rho_{\min }^{c}, 0\right)\left(\int_{\rho_{s}}^{\rho_{\text {min }}^{c}} H^{\prime}(t) d t=0,0<\rho_{\min }^{c}<\right.$ $b)$. The solution of (5.1) through any state on the supersonic loop of the critical trajectory is periodic with period $P_{c}$ (see Figure 4).

Then the following theorem holds.

TheOREM 5.2. Suppose that $0<b<\rho_{s}$ and $\left(\rho_{l}, \alpha\right)$ is on the supersonic loop of critical trajectory, i.e., (5.19) holds, and assume that $\rho_{r}>F\left(\rho_{\min }^{c}\right)$. Let $\bar{E}\left(\rho_{l}\right)=$ $\sqrt{\alpha^{2}+2 \int_{F\left(\rho_{l}\right)}^{\rho_{r}} H^{\prime}(t) d t}$ such that $\left(\rho_{r}, \bar{E}\left(\rho_{l}\right)\right) \in T\left(F\left(\rho_{l}\right), \alpha\right)$. Then

(1) If $L<\ell\left(\left(F\left(\rho_{l}\right), \alpha\right) ;\left(\rho_{r}, \bar{E}\left(\rho_{l}\right)\right)\right)$, then the boundary value problem (1.7) and (1.8) does not have a solution;

(2)If $L=k P_{c}+\ell\left(\left(\rho_{l}, \alpha\right) ;\left(\rho_{s}, 0\right)\right)+\ell\left(\left(\rho_{s}, 0\right) ;\left(\rho_{r}, E_{c}\right)\right)$, where $E_{c}=\sqrt{2 \int_{\rho_{s}}^{\rho_{r}} H^{\prime}(t) d t}$ such that $\left(\rho_{r}, E_{c}\right) \in T\left(\rho_{s}, 0\right)$ and $k \geq 0$ is an integer, then the solution of the boundary value problem (1.7) and (1.8) admits a unique smooth solution;

(3) If $L \geq \ell\left(\left(F\left(\rho_{l}\right), \alpha\right) ;\left(\rho_{r}, \bar{E}\left(\rho_{l}\right)\right)\right)$, but $L \neq k P_{c}+\ell\left(\left(\rho_{l}, \alpha\right) ;\left(\rho_{s}, 0\right)\right)+\ell\left(\left(\rho_{s}, 0\right) ;\left(\rho_{r}, E_{c}\right)\right)$ for some integer $k \geq 0$, then the boundary value problem of (1.7) and (1.8) admits a unique transonic shock solution.

Proof. We only consider the case when $\alpha>0$; the case when $\alpha \leq 0$ can be handled similarly. Set

$$
\begin{gathered}
\bar{E}\left(\rho_{\text {min }}^{c}\right)=\sqrt{2 \int_{F\left(\rho_{\text {min }}^{c}\right)}^{\rho_{r}} H^{\prime}(t) d t}, L_{1}^{c}=\ell\left(\left(\rho_{l}, \alpha\right) ;\left(\rho_{\text {min }}^{c}, 0\right)\right), L_{2}^{c}=\ell\left(\left(\rho_{\text {min }}^{c}, 0\right) ;\left(\rho_{s}, 0\right)\right), \\
L_{3}^{c}=\ell\left(\left(\rho_{\text {min }}^{c}, 0\right) ;\left(\rho_{l}, \alpha\right)\right), L_{4}^{c}=\ell\left(\left(F\left(\rho_{l}\right), \alpha\right) ;\left(\rho_{r}, \bar{E}\left(\rho_{l}\right)\right)\right), \\
L_{5}^{c}=\ell\left(\left(F\left(\rho_{\text {min }}^{c}\right), 0\right) ;\left(\rho_{r}, \bar{E}\left(\rho_{\text {min }}^{c}\right)\right)\right), L_{6}^{c}=\ell\left(\left(\rho_{s}, 0\right) ;\left(\rho_{r}, E_{c}\right)\right) .
\end{gathered}
$$

One notes that

$$
P_{c}=L_{1}^{c}+L_{2}^{c}+L_{3}^{c}
$$


(1) is obvious by the structure of the solutions for (5.1) described in Section 2. If $L \geq L_{4}^{c}$, then there exists an integer $k \geq 0$ such that

$$
k P_{c}+L_{4}^{c} \leq L<(k+1) P_{c}+L_{4}^{c} .
$$

We only need to consider the case when $k=0$. In this case, similar to the proof for Lemma 4.2, the solution can be constructed as follows:

(a) If

$$
L_{4}^{c} \leq L \leq L_{1}^{c}+L_{5}^{c},
$$

then there exist a unique state $\left(\rho_{c}^{*}, E_{c}^{*}\right)$ on supersonic loop of the critical trajectory satisfying $\rho_{\min }^{c} \leq \rho_{c}^{*} \leq \rho_{l}$ and $E_{c}^{*} \geq 0$ and a unique number $\beta^{c}$ satisfying $\left.\bar{E}\left(\rho_{\min }^{c}\right)\right) \leq$ $\beta^{c} \leq \bar{E}\left(\rho_{l}\right)$ such that the following equality holds true:

$$
L=\ell\left(\left(\rho_{l}, \alpha\right) ;\left(\rho_{c}^{*}, E_{c}^{*}\right)\right)+\ell\left(\left(F\left(\rho_{c}^{*}\right), E_{c}^{*}\right) ;\left(\rho_{r}, \beta^{c}\right)\right) .
$$

So the transonic shock location is $a=\ell\left(\left(\rho_{l}, \alpha\right) ;\left(\rho_{c}^{*}, E_{c}^{*}\right)\right)$.

(b) If $L_{1}^{c}+L_{5}^{c} \leq L<L_{1}^{c}+L_{2}^{c}+L_{6}^{c}$, then there exist a unique number $\beta_{1}^{c}$ and a unique state $\left(\rho_{c}^{* *}, E_{c}^{* *}\right)$ on supersonic loop of the critical trajectory satisfying $\rho_{\min }^{c} \leq$ $\rho_{c}^{*}<\rho_{s}$ and $E_{c}^{*} \leq 0$ such that the following equality holds true:

$$
L=L_{1}^{c}+\ell\left(\left(\rho_{\min }^{c}, 0\right) ;\left(\rho_{c}^{* *} E_{c}^{* *}\right)\right)+\ell\left(\left(F\left(\rho_{c}^{* *}\right), E_{c}^{* *}\right) ;\left(\rho_{r}, \beta_{1}^{c}\right)\right) .
$$

Hence the transonic shock location is $a=L_{1}^{c}+\ell\left(\left(\rho_{\min }^{c}, 0\right) ;\left(\rho_{c}^{* *} E_{c}^{* *}\right)\right)$.

(c) If $L=L_{1}^{c}+L_{2}^{c}+L_{6}^{c}$, then the solution of the boundary value problem of (1.7) and (1.8) is smooth (no transonic shock). In the $(\rho, E)$-phase plane, the solution starts from $\left(\rho_{l}, \alpha\right)$, travels along the supersonic loop of the critical trajectory to the sonic state $\left(\rho_{s}, 0\right)$, then travels along the subsonic branch of the critical trajectory to the state $\left(\rho_{r}, E_{c}\right)$.

(d) If $L_{1}^{c}+L_{2}^{c}+L_{6}^{c}<L \leq L_{1}^{c}+L_{2}^{c}+L_{3}^{c}+L_{4}^{c}$, then there exist a unique number $\beta_{2}^{c}$ and a unique state $\left(\rho_{c}^{0}, E_{c}^{0}\right)$ on the supersonic loop of the critical trajectory satisfying $\rho_{l} \geq \rho_{c}^{0}<\rho_{s}$ and $E_{c}^{0}>0$ such that the following equality holds true:

$$
L=L_{1}^{c}+L_{2}^{c}+\ell\left(\left(\rho_{s}, 0\right) ;\left(\rho_{c}^{0}, E_{c}^{0}\right)\right)+\ell\left(\left(F\left(\rho_{c}^{0}\right), E_{c}^{0}\right) ;\left(\rho_{r}, \beta_{2}^{c}\right)\right) .
$$

Thus the transonic shock location is $a=L_{1}^{c}+L_{2}^{c}+\ell\left(\left(\rho_{s}, 0\right) \cdot\left(\rho_{c}^{0}, E_{c}^{0}\right)\right)$. This finishes the proof of the Theorem.

When the state $\left(\rho_{l}, \alpha\right)$ is outside the supersonic loop of the critical trajectory, i.e.

$$
\frac{1}{2} \alpha^{2}-\int_{\rho_{s}}^{\rho_{l}} H^{\prime}(s) d s>0, \text { and } 0<\rho_{l}<\rho_{s},
$$

the solutions can be constructed as follows. Let $T_{o}$ the supersonic trajectory passing through the point $\left(\rho_{l}, \alpha\right)$ on $(\rho, E)$ phase plane, i.e.,

$$
T_{o}=\left\{(\rho, E): \frac{1}{2} E^{2}=\frac{1}{2} \alpha^{2}+\int_{\rho_{l}}^{\rho} H^{\prime}(t) d t, 0<\rho<\rho_{s}\right\},
$$

and $T_{o}^{\text {shock }}$ be the shock conjugate of $T_{o}$,

$$
T_{o}^{\text {shock }}=\left\{(F(\rho), E):(\rho, E) \in T_{o}\right\} .
$$


Then $T_{o}^{\text {shock }}$ intersects the subsonic branch of the critical trajectory at two points, denoted by $(\check{\rho}, \breve{E})$ and $(\check{\rho},-\check{E})$, where $\breve{E}>0$. Denote the intersection point of $T_{o}$ with $\rho$-axis by $\left(\rho_{\min }^{o}, 0\right)$. Then we have the following theorem:

THEOREM 5.3. Suppose that $0<b<\rho_{s}$ and $\left(\rho_{l}, \alpha\right)$ is outside the supersonic loop of critical trajectory, i.e., (5.24) holds, and assume that $\rho_{r}>F\left(\rho_{\min }^{c}\right)$. Then

(1) if $\quad \alpha \geq-\check{E}$ and if $L<\ell\left(\left(F\left(\rho_{l}\right), \alpha\right) ;\left(\rho_{r}, \bar{E}\left(\rho_{l}\right)\right)\right)$, where $\bar{E}\left(\rho_{l}\right)=$ $\sqrt{\alpha^{2}+2 \int_{\rho_{l}}^{\rho_{r}} H^{\prime}(s) d s}$, or $\alpha<-\check{E}$, then the boundary value problem (1.7) and (1.8) does not have a solution;

(2) if $\alpha \geq-\check{E}$ and $L \geq \ell\left(\left(F\left(\rho_{l}\right), \alpha\right) ;\left(\rho_{r}, \bar{E}\left(\rho_{l}\right)\right)\right)$, then the boundary value problem of (1.7) and (1.8) admits a unique transonic shock solution.

Proof. (1) follows easily by a phase plane analysis. To prove (2), we assume that $\alpha>0$ (the proof for $-\check{E} \leq \alpha \leq 0$ is similar). Define

$$
\bar{L}(\rho)=\ell\left(\left(\rho_{l}, \alpha\right) ;(\rho, E(\rho))\right)+\ell\left((F(\rho), E(\rho)) ;\left(\rho_{r}, \bar{E}(\rho)\right)\right), \text { for }(\rho, E(\rho)) \in T_{o}, E(\rho) \geq 0,
$$

where $\bar{E}(\rho)=\sqrt{E^{2}(\rho)+2 \int_{F(\rho)}^{\rho_{r}} H^{\prime}(t) d t}$,

$$
\tilde{L}(\rho)=\ell\left(\left(\rho_{\min }^{o}, 0\right) ;(\rho, E(\rho))\right)+\ell\left((F(\rho), E(\rho)) ;\left(\rho_{r}, \bar{E}(\rho)\right)\right),
$$

for $\quad(\rho, E(\rho)) \in T_{o}, \quad \rho_{\min }^{o} \leq \rho<F^{-1}(\check{\rho}), \quad E(\rho)<0, \quad$ where $\quad \bar{E}(\rho)=$ $\sqrt{E^{2}(\rho)+2 \int_{F(\rho)}^{\rho_{r}} H^{\prime}(t) d t}$. It follows from the same argument as in the proof of Lemmas 4.2 and 4.3 that $\bar{L}(\rho)$ is a strictly decreasing function of $\rho$ for $\rho_{\min }^{o} \leq \rho \leq \rho_{l}$ and $\tilde{L}(\rho)$ is a strictly increasing function of $\rho$ for $\rho_{\min }^{o} \leq \rho<F^{-1}(\check{\rho})$. There are three cases to consider.

(a) If $\bar{L}\left(\rho_{l}\right) \leq L \leq \bar{L}\left(\rho_{\text {min }}^{o}\right)$, then there exits a unique state $\left(\rho_{1}, E_{1}\right) \in T_{o}$ with $E_{1} \geq$ 0 such that $L=\bar{L}\left(\rho_{1}\right)$. In this case, the transonic shock location is at $a=$ $\ell\left(\left(\rho_{l}, \alpha\right) ;\left(\rho_{1}, E_{1}\right)\right)$.

(b). If $\ell\left(\left(\rho_{l}, \alpha\right) ;\left(\rho_{\text {min }}^{o}, 0\right)\right)+\tilde{L}\left(\rho_{\text {min }}^{o}\right) \leq L<\ell\left(\left(\rho_{l}, \alpha\right) ;\left(\rho_{\text {min }}^{o}, 0\right)\right)+\lim _{\rho \rightarrow F^{-1}(\check{\rho})} \tilde{L}(\rho)$, then there exits a unique state $\left(\rho_{2}, E_{2}\right) \in T_{o}$ with $-\check{E}<E_{2} \leq 0$ such that $L=\tilde{L}\left(\rho_{1}\right)$. In this case, the transonic shock location is at $a=\ell\left(\left(\rho_{l}, \alpha\right) ;\left(\rho_{2}, E_{2}\right)\right)$.

(c) If $L \geq \ell\left(\left(\rho_{l}, \alpha\right) ;\left(\rho_{\text {min }}^{o}, 0\right)\right)+\lim _{\rho \rightarrow F^{-1}(\check{\rho})} \tilde{L}(\rho)$, the only possible solution of the boundary value problem is described as follows: in the $(\rho, E)$-phase plane, the solution starting from $\left(\rho_{l}, \alpha\right)$ travels along $T_{o}$ counterclockwise and can not travel beyond the point $\left(F^{-1}(\check{\rho}),-\check{E}\right)$. Since otherwise, it travels beyond the point $\left(F^{-1}(\check{\rho}),-\check{E}\right)$ and can never reach the state $\rho_{r}$. So the solution travels along the $T_{o}$ and reaches the point $\left(F^{-1}(\check{\rho}),-\check{E}\right)$, and jumps to the point $(\check{\rho},-\check{E})$ by a transonic shock. From $(\check{\rho},-\check{E})$ the solution travels along the lower portion of the subsonic branch of the critical trajectory $\left\{(\rho, E): E=-\sqrt{2 \int_{\rho_{s}}^{\rho} H^{\prime}(t) d t}, \rho>\rho_{s}\right\}$ and reaches the sonic point $\left(\rho_{s}, 0\right)$, and from the sonic point $\left(\rho_{s}, 0\right)$ the solution travels along the supersonic loop $\left\{(\rho, E): \frac{1}{2} E^{2}=\int_{\rho_{s}}^{\rho} H^{\prime}(t) d t, \rho<\rho_{s}\right\} k$ times $k=0,1,2, \ldots$ and comes back to the sonic point, where the value of $k$ is determined by $L$. Then from the sonic point, the solution travels along the upper portion of the subsonic branch of the critical trajectory $\left\{(\rho, E): E=\sqrt{2 \int_{\rho_{s}}^{\rho} H^{\prime}(t) d t}, \rho>\rho_{s}\right\}$ in the direction that $\rho$ increases and reaches the state $\left(\rho_{r}, E_{c}\right)$ where $E_{c}=\sqrt{2 \int_{\rho_{s}}^{\rho_{r}} H^{\prime}(t) d t}$. 


\section{Transonic shock solutions for the case when $b>\rho_{s}$.}

In this section, we study the case when $b>\rho_{s}$, i.e., $b$ is in the subsonic region. It is easy to see that

$$
H^{\prime}(\rho)>0 \text { for } 0<\rho<\rho_{s} \text { and } \rho>b, H^{\prime}(\rho)<0 \text {, for } \rho_{s}<\rho<b .
$$

First, we study the geometry of the shock curves in the $(\rho, E)$-plane in the following two lemmas. Let

$$
T_{b}:=\left\{(\rho, E): \frac{1}{2} E^{2}-H(\rho)=-H\left(F^{-1}(b)\right), \rho \leq \rho_{s}\right\},
$$

and $S_{b}$ be the set of states which can be connected to the states of $T_{b}$ by transonic shocks, i.e.,

$$
S_{b}:=\left\{(F(\rho), E):(\rho, E) \in T_{b}\right\} .
$$

Then $S_{b}$ is a curve in the $(\rho, E)$-plane satisfying the following equation:

$$
\frac{1}{2} E^{2}-H\left(F^{-1}(\rho)\right)=-H\left(F^{-1}(b)\right), \quad \rho_{s} \leq \rho \leq b .
$$

Clearly $(b, 0) \in S_{b}$. Let

$$
C_{b}^{s u b}:=\left\{(\rho, E): \frac{1}{2} E^{2}-H(\rho)=-H(b), \rho_{s} \leq \rho \leq b\right\},
$$

the subsonic branch of the critical trajectory passing through $(b, 0)$. Then the following lemma holds (see Figure 5).

Lemma 6.1. Suppose that the pressure function $p$ satisfies (1.3) and $b>\rho_{s}$. Then

$$
H\left(F^{-1}(\rho)\right)-H\left(F^{-1}(b)\right)>H(\rho)-H(b), \quad \rho_{s} \leq \rho<b,
$$

i.e., the curve $S_{b}$ is outside the curve $C_{b}^{s u b}$.

Proof. Let $\rho_{b}$ be the density satisfying

$$
0<\rho_{b}<\rho_{s}, \quad H\left(\rho_{b}\right)=H(b) .
$$

We first show that

$$
\rho_{s}<F\left(\rho_{b}\right)<b .
$$

Since $H\left(\rho_{b}\right)=H(b)$, it follows from the definition of the function $H$ that

$$
\begin{aligned}
(p(b) & \left.+\frac{J^{2}}{b}\right)-\left(p\left(\rho_{b}\right)+\frac{J^{2}}{\rho_{b}}\right) \\
& -\int_{\rho_{b}}^{b}\left(p^{\prime}(t)-\frac{J^{2}}{t^{2}}\right) \frac{b}{t} d t=0 .
\end{aligned}
$$

Let

$$
\begin{aligned}
f(z):= & \left(p(z)+\frac{J^{2}}{z}\right)-\left(p(g(z))+\frac{J^{2}}{g(z)}\right) \\
& -\int_{g(z)}^{z}\left(p^{\prime}(t)-\frac{J^{2}}{t^{2}}\right) \frac{z}{t} d t
\end{aligned}
$$


for $z \geq \rho_{s}$, where $g(z)=F^{-1}(z)$. Since $g\left(\rho_{s}\right)=\rho_{s}$, one has

$$
f\left(\rho_{s}\right)=0 .
$$

On the other hand,

$$
p(g(z))+\frac{J^{2}}{g(z)}=p(z)+\frac{J^{2}}{z}, \quad z \geq \rho_{s}
$$

Hence,

$$
\left(p^{\prime}(g(z))-\frac{J^{2}}{(g(z))^{2}}\right) g^{\prime}(z)=p^{\prime}(z)-\frac{J^{2}}{z^{2}}, \quad z \geq \rho_{s} .
$$

(6.8) and (6.10) yield

$$
\begin{aligned}
f^{\prime}(z) & =\left(p^{\prime}(z)-\frac{J^{2}}{z^{2}}\right) \frac{1}{g(z)}(z-g(z))-\int_{g(z)}^{z}\left(p^{\prime}(t)-\frac{J^{2}}{t^{2}}\right) \frac{1}{t} d t \\
& =\int_{g(z)}^{z}\left(p^{\prime}(z)-\frac{J^{2}}{z^{2}}\right) \frac{1}{g(z)} d t-\int_{g(z)}^{z}\left(p^{\prime}(t)-\frac{J^{2}}{t^{2}}\right) \frac{1}{t} d t .
\end{aligned}
$$

Since $p^{\prime \prime}(\rho)>0$ for $\rho>0$ (see (1.3)), it follows that

$$
p^{\prime}(z)-\frac{J^{2}}{z^{2}}>p^{\prime}(t)-\frac{J^{2}}{t^{2}}, \text { for } g(z) \leq t<z .
$$

This, together with (6.11), implies

$$
f^{\prime}(z)>0, \text { for } z>\rho_{s} .
$$

Therefore, in view of (6.9), one has

$$
f(b)>0
$$

since $b>\rho_{s}$. This means, in view of (6.8),

$$
\begin{aligned}
(p(b)+ & \left.\frac{J^{2}}{b}\right)-\left(p(g(b))+\frac{J^{2}}{g(b)}\right) \\
& -\int_{g(b)}^{b}\left(p^{\prime}(t)-\frac{J^{2}}{t^{2}}\right) \frac{b}{t} d t>0 .
\end{aligned}
$$

Next, we define

$$
\begin{aligned}
q(\rho):= & \left(p(b)+\frac{J^{2}}{b}\right)-\left(p(\rho)+\frac{J^{2}}{\rho}\right) \\
& -\int_{\rho}^{b}\left(p^{\prime}(t)-\frac{J^{2}}{t^{2}}\right) \frac{b}{t} d t, \text { for } 0<\rho<\rho_{s} .
\end{aligned}
$$

It is easy to verify that

$$
q^{\prime}(\rho)=\left(p^{\prime}(\rho)-\frac{J^{2}}{\rho^{2}}\right)\left(\frac{b}{\rho}-1\right)<0, \text { for } 0<\rho<\rho_{s},
$$


since $b>\rho_{s}>\rho$. This, together with (6.7) and (6.13), implies

$$
\rho_{b}<g(b)=F^{-1}(b)
$$

Since $F^{\prime}(\rho)<0$ for $0<\rho<\rho_{s}$ (cf. 4.2), (6.6) follows.

Next, let

$$
h(\rho)=H\left(F^{-1}(\rho)\right)-H(\rho)+H(b)-H\left(F^{-1}(b)\right), \quad \rho_{s} \leq \rho<b .
$$

Since $F^{-1}\left(\rho_{s}\right)=\rho_{s}$, it holds that

$$
h\left(\rho_{s}\right)=H(b)-H\left(F^{-1}(b)\right)=H\left(\rho_{b}\right)-H\left(F^{-1}(b)\right),
$$

where $\rho_{b}<\rho_{s}$ is the constant defined in (6.5). Since $\rho_{s}<b, H^{\prime}(\rho)>0$ for $0<\rho<\rho_{s}$. Thus, (6.16) and (6.17) imply

$$
h\left(\rho_{s}\right)>0 .
$$

On the other hand, just as in (6.10), one has

$$
\left(p^{\prime}(g(\rho))-\frac{J^{2}}{(g(\rho))^{2}}\right) g^{\prime}(\rho)=p^{\prime}(\rho)-\frac{J^{2}}{\rho^{2}}, \quad \rho \geq \rho_{s},
$$

where and in the following

$$
g(\rho)=F^{-1}(\rho) .
$$

This gives

$$
H^{\prime}(g(\rho)) g^{\prime}(\rho)=\left(p^{\prime}(\rho)-\frac{J^{2}}{\rho^{2}}\right)\left(\frac{g(\rho)-b}{g(\rho)}\right), \quad \rho \geq \rho_{s} .
$$

Therefore,

$$
h^{\prime}(\rho)=\left(p^{\prime}(\rho)-\frac{J^{2}}{\rho^{2}}\right)\left(\frac{b}{\rho}-\frac{b}{g(\rho)}\right), \quad \rho \geq \rho_{s} .
$$

Since $g(\rho)=F^{-1}(\rho)<\rho_{s}$ for $\rho>\rho_{s}$, it follows that

$$
h^{\prime}(\rho)<0, \quad \rho>\rho_{s} .
$$

On the other hand

$$
h(b)=0 .
$$

This, together with (6.18) and (6.22), implies (6.4).

For $\left(\rho_{l}, \alpha\right)$ satisfying $0<\rho_{l}<\rho_{s}$, let

$$
\bar{T}\left(\rho_{l}, \alpha\right)=\left\{(\rho, E): \frac{1}{2} E^{2}-H(\rho)=\frac{1}{2} \alpha^{2}-H\left(\rho_{l}\right), \rho>0\right\},
$$

and

$$
S\left(\rho_{l}, \alpha\right)=\left\{(\rho, E): \frac{1}{2} E^{2}-H\left(F^{-1}(\rho)\right)=\frac{1}{2} \alpha^{2}-H\left(\rho_{l}\right), \rho \geq \rho_{s}\right\} .
$$


So $S\left(\rho_{l}, \alpha\right)$ is the set of states which can be connected to the set $\left\{(\rho, E) \in \bar{T}\left(\rho_{l}, \alpha\right)\right.$ : $\left.0<\rho \leq \rho_{s}\right\}$ by a transonic shock. Moreover, we define

$$
\begin{gathered}
E_{1}^{2}(\rho)=\alpha^{2}+2\left(H(\rho)-H\left(\rho_{l}\right)\right), \\
E_{2}^{2}(\rho)=\alpha^{2}+2\left(H\left(F^{-1}(\rho)\right)-H\left(\rho_{l}\right)\right), \rho_{s} \leq \rho \leq \rho^{\alpha},
\end{gathered}
$$

where $\rho^{\alpha}$ is determined by

$$
H\left(F^{-1}\left(\rho^{\alpha}\right)\right)=H\left(\rho_{l}\right)-\frac{1}{2} \alpha^{2}, \rho^{\alpha}>\rho_{s} .
$$

Obviously

$$
E_{2}\left(\rho^{\alpha}\right)=0
$$

Then we have following lemma.

Lemma 6.2. Suppose the pressure function p satisfies (1.3) and $b>\rho_{s}$. Then

$$
E_{1}^{2}(\rho)>E_{2}^{2}(\rho), \quad \text { for } \rho_{s}<\rho \leq \rho^{\alpha},
$$

i.e., the curve $\tilde{T}\left(\rho_{l}, \alpha\right)$ is outside the shock curve $S\left(\rho_{l}, \alpha\right)$ in the $(\rho, E)$-plane.

Proof. Obviously

$$
E_{1}^{2}\left(\rho_{s}\right)=E_{2}^{2}\left(\rho_{s}\right)
$$

Let $g(\rho)=F^{-1}(\rho)$ for $\rho \geq \rho_{s}$. By (4.2), we have

$$
p^{\prime}(g(\rho))-\frac{J^{2}}{(g(\rho))^{2}} g^{\prime}(\rho)=p^{\prime}(\rho)-\frac{J^{2}}{\rho^{2}}, \rho \geq \rho_{s} .
$$

It follows from (6.26), (6.27), and (6.32) that

$$
\begin{aligned}
& \frac{d\left(E_{1}^{2}(\rho)-E_{2}^{2}(\rho)\right)}{d \rho} \\
= & 2\left(H^{\prime}(\rho)-H^{\prime}(g(\rho)) g^{\prime}(\rho)\right) \\
= & 2\left(p^{\prime}(\rho)-\frac{J^{2}}{\rho^{2}}\right)\left(\frac{b}{g(\rho)}-\frac{b}{\rho}\right),
\end{aligned}
$$

for $\quad \rho_{s} \leq \rho \leq \rho^{\alpha} . \quad$ For $\quad \rho>\rho_{s}, \quad g(\rho)=F^{-1}(\rho)<\rho_{s}, \quad p^{\prime}(\rho)-\frac{J^{2}}{\rho^{2}}>0 . \quad$ Therefore, $\frac{d\left(E_{1}^{2}(\rho)-E_{2}^{2}(\rho)\right)}{d \rho}>0$ for $\rho_{s}<\rho \leq \rho^{\alpha}$. This, together with (6.31), implies (6.30).

In the following, it is always assumed that the pressure function $p$ satisfies (1.3) and $b>\rho_{s}$. Denote by

$$
\mathfrak{T}\left(\rho_{l}, \alpha\right):=\left\{(\rho, E): \frac{1}{2} E^{2}-H(\rho)=\frac{1}{2} \alpha^{2}-H\left(\rho_{l}\right), \rho_{\text {min }}^{\text {out }} \leq \rho<\rho_{s}\right\}
$$

the supersonic trajectory passing through $\left(\rho_{l}, \alpha\right)$, and by

$$
S\left(\rho_{l}, \alpha\right):=\left\{\left(F(\rho, E):(\rho, E) \in \mathfrak{T}\left(\rho_{l}, \alpha\right)\right\}\right.
$$


the curve on the $(\rho, E)$-plane consisting of the states which can be connected to those on $\mathfrak{T}\left(\rho_{l}, \alpha\right)$ by a transonic shock. In the case when the curve $S\left(\rho_{l}, \alpha\right)$ intersects the critical trajectory passing through $(b, 0)$ at two points, we denote those two points by $\left(\rho_{c}, E_{c}\right)$ and $\left(\rho_{c},-E_{c}\right)$ with $E_{c}>0$. It follows from Lemmas 6.1 and 6.2 , the geometry of the shock curves and the phase portrait of system (1.7) are as shown in Figures 6-16 for the different cases. With this, we can construct transonic shock solutions according to the different situations of $\left(\rho_{l}, \alpha\right), \rho_{r}$, and $L$. This will be done in the following subsections.

6.1. Case 1: $\left(\rho_{l}, \alpha\right)$ is outside the trajectory through $\left(F^{-1}(b), 0\right)$. In this case,

$$
\frac{1}{2} \alpha^{2}-H\left(\rho_{l}\right)>-H\left(F^{-1}(b)\right), 0<\rho_{l}<\rho_{s} .
$$

Define $\rho_{\text {min }}^{\text {out }}$ by

$$
H\left(\rho_{m i n}^{\text {out }}\right)=H\left(\rho_{l}\right)-\frac{1}{2} \alpha^{2}, 0<\rho_{\text {min }}^{\text {out }}<\rho_{s}
$$

so that $\left(\rho_{\text {min }}^{\text {out }}, 0\right)$ is the intersection point of the trajectory through $(\rho, \alpha)$ and the line $E=0$. There are the following subcases to consider.

\section{Subcase 1.}

$$
\rho_{r} \geq F\left(\rho_{\text {min }}^{\text {out }}\right),
$$

the phase portrait of this case is given in Figure 6;

\section{Subcase 2.}

$$
\rho_{c} \leq \rho_{r} \leq F\left(\rho_{\text {min }}^{\text {out }}\right),
$$

the phase portrait of this case is given in Figure 7;

Subcase 3.

$$
b<\rho_{r}<\rho_{c},
$$

the phase portrait of this case is given in Figure 8;

\section{Subcase 4.}

$$
\rho_{s}<\rho_{r}<b,
$$

the phase portrait of this case is given in Figure 9. The analysis for Subcase 1 is simple. We give more detailed analysis for subcases 2-4.

Analysis for Subcase 2. In Subcases 2, (6.39), the line $\rho=\rho_{r}$ intersects the shock curve $S\left(\rho_{l}, \alpha\right)$ at two points $\left(\rho_{r}, E_{r}^{0}\right)$ and $\left(\rho_{r},-E_{r}^{0}\right)$ with $E_{r}^{0}>0$, the trajectory passing through $\left(\rho_{r}, 0\right)$ satisfying $\frac{1}{2} E^{2}-H(\rho)=-H\left(\rho_{r}\right)$ intersects the shock curve $S\left(\rho_{l}, \alpha\right)$ at two points $\left(\rho_{r}^{1}, E_{r}^{1}\right)$ and $\left(\rho_{r}^{1},-E_{r}^{1}\right)$ with $E_{r}^{1}>0$ (see Figure 7). Clearly, $\rho_{r}^{1}>\rho_{r}$ and $E_{r}^{0}>E_{r}^{1}$. For $\bar{\rho} \in\left[F^{-1}\left(\rho_{r}^{1}\right), F^{-1}\left(\rho_{c}\right)\right]$, set

$$
E_{r}(\bar{\rho})=\sqrt{E^{2}(\bar{\rho})+2\left(\left(H\left(\rho_{r}\right)-H(F(\bar{\rho}))\right.\right.},
$$

where

$$
E(\bar{\rho})=-\sqrt{\alpha^{2}+2\left(H(\bar{\rho})-H\left(\rho_{l}\right)\right)}
$$


satisfies $(\bar{\rho}, E(\bar{\rho})) \in T\left(\rho_{\text {min }}^{\text {out }}, 0\right) \subset T\left(\rho_{l}, \alpha\right)$. In this case, for any state $(\bar{\rho}, E(\bar{\rho})) \in T\left(\rho_{l}, \alpha\right)$ satisfying $F^{-1}\left(\rho_{r}^{1}\right)<\bar{\rho}<F^{-1}\left(\rho_{c}\right),-E_{c}<E(\bar{\rho})<-E_{r}^{1}$, the trajectory $T(F(\bar{\rho}), E(\bar{\rho}))$ starting from $(F(\bar{\rho}), E(\bar{\rho}))$ intersects the line $\rho=\rho_{r}$ twice at $\left(\rho_{r},-E_{r}(\bar{\rho})\right)$ and $\left(\rho_{r}, E_{r}(\bar{\rho})\right)$. Obviously,

$$
E_{r}\left(F^{-1}\left(\rho_{r}^{1}\right)\right)=0, E_{r}\left(F^{-1}\left(\rho_{r}\right)\right)=E_{r}^{0} .
$$

For $(\bar{\rho}, E(\bar{\rho})) \in T\left(\rho_{\text {min }}^{\text {out }}, 0\right) \subset T\left(\rho_{l}, \alpha\right)$, define

$$
\begin{gathered}
Y(\bar{\rho})=\ell\left(\left(\rho_{l}, \alpha\right) ;(\bar{\rho}, E(\bar{\rho}))\right)+\ell\left((F(\bar{\rho}), E(\bar{\rho})) ;\left(\rho_{r},-E_{r}(\bar{\rho})\right)\right), \\
\quad \text { for } \bar{\rho} \in\left[F^{-1}\left(\rho_{r}^{1}\right), F^{-1}\left(\rho_{r}\right)\right],-E_{r}^{0} \leq E(\bar{\rho}) \leq-E_{r}^{1} \\
Z(\bar{\rho})=\ell\left(\left(\rho_{l}, \alpha\right) ;(\bar{\rho}, E(\bar{\rho}))\right)+\ell\left((F(\bar{\rho}), E(\bar{\rho})) ;\left(\rho_{r}, E_{r}(\bar{\rho})\right)\right) \\
\quad \text { for } \bar{\rho} \in\left[F^{-1}\left(\rho_{r}^{1}\right), F^{-1}\left(\rho_{c}\right)\right),-E_{c}<E(\bar{\rho}) \leq-E_{r}^{1} .
\end{gathered}
$$

It should be noted that $Z(\bar{\rho})=Y(\bar{\rho})+\ell\left(\left(\rho_{r},-E_{r}(\bar{\rho})\right) ;\left(\rho_{r}, E_{r}(\bar{\rho})\right)\right)$ for $\bar{\rho} \in$ $\left[F^{-1}\left(\rho_{r}^{1}\right), F^{-1}\left(\rho_{r}\right)\right]$ and $Y\left(F^{-1}\left(\rho_{r}^{1}\right)\right)=Z\left(F^{-1}\left(\rho_{r}^{1}\right)\right)$. With those notations, we have the following Lemma,

Lemma 6.3. Suppose that $\left(\rho_{l}, \alpha\right)$ satisfies (6.36), $\rho_{r}$ satisfies (6.39), and $\alpha>E_{r}^{0}$. Then there exists a unique state $(\hat{\rho}, E(\hat{\rho})) \in T\left(\rho_{l}, \alpha\right)$ satisfying $F^{-1}\left(\rho_{r}^{1}\right)<\hat{\rho}<F^{-1}\left(\rho_{r}\right)$ and $-E_{r}^{0}<E(\hat{\rho})<-E_{r}^{1}$ such that

$$
Y^{\prime}\left(F^{-1}\left(\rho_{r}^{1}\right)\right)=-\infty,\left\{\begin{array}{l}
Y^{\prime}(\bar{\rho})<0, \text { for } F^{-1}\left(\rho_{r}^{1}\right)<\bar{\rho}<\hat{\rho}, \\
Y^{\prime}(\bar{\rho})>0, \text { for } \hat{\rho}<\bar{\rho} \leq F^{-1}\left(\rho_{r}\right) .
\end{array}\right.
$$

So

$$
Y(\hat{\rho})=\min _{F^{-1}\left(\rho_{r}^{1}\right) \leq \bar{\rho} \leq F^{-1}\left(\rho_{r}\right)} Y(\bar{\rho})
$$

The proof of this Lemma will be given in the Appendix.

Analysis for Subcase 3. In Subcase 3, (6.40), the line $\rho=\rho_{r}$ intersects the shock curve $S\left(\rho_{l}, \alpha\right)$ at two points $\left(\rho_{r}, E_{r}\right)$ and $\left(\rho_{r},-E_{r}\right)$ with $E_{r}>0$, and the trajectory passing through $\left(\rho_{r}, 0\right)$ satisfying $\frac{1}{2} E^{2}-H(\rho)=-H\left(\rho_{r}\right)$ intersects the shock curve $S\left(\rho_{l}, \alpha\right)$ at two points $\left(\bar{\rho}_{r}, \bar{E}_{r}\right)$ and $\left(\bar{\rho}_{r},-\bar{E}_{r}\right)$ with $\bar{E}_{r}>0$ (see Figure 8). Clearly, $\bar{\rho}_{r}>\rho_{r}$ and $E_{r}>\bar{E}_{r}$.

For $\bar{\rho} \in\left[F^{-1}\left(\bar{\rho}_{r}\right), F^{-1}\left(\rho_{r}\right)\right]$, let

$$
E_{r}(\bar{\rho})=\sqrt{E^{2}(\bar{\rho})+2\left(H\left(\rho_{r}\right)-H(F(\bar{\rho}))\right)},
$$

where $E(\bar{\rho})=-\sqrt{\alpha^{2}+2\left(H(\bar{\rho})-H\left(\rho_{l}\right)\right)}$ satisfies $(\bar{\rho}, E(\bar{\rho})) \in T\left(\rho_{\text {min }}^{\text {out }}, 0\right) \subset T\left(\rho_{l}, \alpha\right)$.

In this case, for any state $\left(\bar{\rho}, E(\bar{\rho}) \in T\left(\rho_{l}, \alpha\right)\right.$ satisfying $F^{-1}\left(\bar{\rho}_{r}\right)<\bar{\rho}<F^{-1}\left(\rho_{c}\right)$, $-E_{c}<E(\bar{\rho})<-\bar{E}_{r}$, the trajectory $T(F(\bar{\rho}), E(\bar{\rho}))$ starting from $(F(\bar{\rho}), E(\bar{\rho}))$ intersects the line $\rho=\rho_{r}$ twice at $\left(\rho_{r},-E_{r}(\bar{\rho})\right)$ and $\left(\rho_{r}, E_{r}(\bar{\rho})\right)$. Obviously, $E_{r}\left(F^{-1}\left(\bar{\rho}_{r}\right)\right)=$ $0, E_{r}\left(F^{-1}\left(\rho_{c}\right)\right)=E_{c}$. For $(\bar{\rho}, E(\bar{\rho})) \in T\left(\rho_{\text {min }}^{\text {out }}, 0\right) \subset T\left(\rho_{l}, \alpha\right)$, define

$$
\begin{gathered}
\bar{Y}(\bar{\rho})=\ell\left(\left(\rho_{l}, \alpha\right) ;(\bar{\rho}, E(\bar{\rho}))\right)+\ell\left((F(\bar{\rho}), E(\bar{\rho})) ;\left(\rho_{r},-E_{r}(\bar{\rho})\right)\right), \\
\text { for } \bar{\rho} \in\left[F^{-1}\left(\bar{\rho}_{r}\right), F^{-1}\left(\rho_{r}\right)\right],-E_{r} \leq E(\bar{\rho}) \leq-\bar{E}_{r},
\end{gathered}
$$




$$
\begin{aligned}
\bar{Z}(\bar{\rho})= & \ell\left(\left(\rho_{l}, \alpha\right) ;(\bar{\rho}, E(\bar{\rho}))\right)+\ell\left((F(\bar{\rho}), E(\bar{\rho})) ;\left(\rho_{r}, E_{r}(\bar{\rho})\right)\right), \\
& \text { for } \bar{\rho} \in\left[F^{-1}\left(\bar{\rho}_{r}\right), F^{-1}\left(\rho_{c}\right)\right),-E_{c}<E(\bar{\rho}) \leq-\bar{E}_{r} .
\end{aligned}
$$

It should be noted that $\bar{Z}(\bar{\rho})=\bar{Y}(\bar{\rho})+\ell\left(\left(\rho_{r},-E_{r}(\bar{\rho})\right) ;\left(\rho_{r}, E_{r}(\bar{\rho})\right)\right)$ for $\bar{\rho} \in$ $\left[F^{-1}\left(\bar{\rho}_{r}\right), F^{-1}\left(\rho_{c}\right)\right]$ and $\bar{Y}\left(F^{-1}\left(\bar{\rho}_{r}\right)\right)=\bar{Z}\left(F^{-1}\left(\bar{\rho}_{r}\right)\right)$. Similar to Lemma 6.3, we have

LEMmA 6.4. Suppose that $\left(\rho_{l}, \alpha\right)$ satisfies (6.36), (6.40) holds, and $\alpha>E_{r}^{0}$. Then there exists a unique state $(\hat{\rho}, E(\hat{\rho})) \in T\left(\rho_{l}, \alpha\right)$ satisfying $F^{-1}\left(\bar{\rho}_{r}\right)<\hat{\rho}<F^{-1}\left(\rho_{c}\right)$ and $-E_{r}<E(\hat{\rho})<-E_{c}$ such that

$$
\bar{Y}^{\prime}\left(F^{-1}\left(\bar{\rho}_{r}\right)\right)=-\infty,\left\{\begin{array}{l}
\bar{Y}^{\prime}(\bar{\rho})<0, \text { for } F^{-1}\left(\bar{\rho}_{r}\right)<\bar{\rho}<\hat{\rho}, \\
\bar{Y}^{\prime}(\bar{\rho})>0, \text { for } \hat{\rho}<\bar{\rho} \leq F^{-1}\left(\rho_{r}\right) .
\end{array}\right.
$$

So

$$
\bar{Y}(\hat{\rho})=\min _{F^{-1}\left(\bar{\rho}_{r}\right) \leq \bar{\rho} \leq F^{-1}\left(\rho_{c}\right)} \bar{Y}(\bar{\rho}) .
$$

The proof of this lemma is almost the same as that for Lemma 6.3 given in the Appendix, so we omit it.

Analysis for Subcase 4. In Subcase 4, (6.41), the line $\rho=\rho_{r}$ intersects the shock curve $S\left(\rho_{l}, \alpha\right)$ at two points $\left(\rho_{r}, E_{r}\right)$ and $\left(\rho_{r},-E_{r}\right)$ with $E_{r}>0$. The trajectory passing through $(b, 0)$ satisfying $\frac{1}{2} E^{2}-H(\rho)=-H(b)$ intersects the shock curve $S\left(\rho_{l}, \alpha\right)$ at two points $\left(\rho_{c}, E_{c}\right)$ and $\left(\rho_{c},-E_{c}\right)$ with $E_{c}>0$ (see Figure 9$)$. For $\bar{\rho} \in\left[F^{-1}\left(\rho_{c}\right), F^{-1}\left(\rho_{r}\right)\right]$, set

$$
e_{r}(\bar{\rho})=-\sqrt{E^{2}(\bar{\rho})+2\left(\left(H\left(\rho_{r}\right)-H(F(\bar{\rho}))\right.\right.},
$$

where $E(\bar{\rho})=-\sqrt{\alpha^{2}+2\left(H(\bar{\rho})-H\left(\rho_{l}\right)\right)}$ satisfies $(\bar{\rho}, E(\bar{\rho})) \in T\left(\rho_{\text {min }}^{\text {out }}, 0\right) \subset T\left(\rho_{l}, \alpha\right)$. For $(\bar{\rho}, E(\bar{\rho})) \in T\left(\rho_{\text {min }}^{\text {out }}, 0\right) \subset T\left(\rho_{l}, \alpha\right)$, we define

$$
\mu(\bar{\rho})=\ell\left(\left(\rho_{l}, \alpha\right) ;(\bar{\rho}, E(\bar{\rho}))\right)+\ell\left((F(\bar{\rho}), E(\bar{\rho})) ;\left(\rho_{r},-e_{r}(\bar{\rho})\right)\right)
$$

for $\bar{\rho} \in\left[F^{-1}\left(\rho_{c}\right), F^{-1}\left(\rho_{r}\right)\right],-E_{r} \leq E(\bar{\rho}) \leq-E_{c}$. Then the following lemma holds.

LEMma 6.5. Suppose that $\left(\rho_{l}, \alpha\right)$ satisfies (6.36), (6.41) holds, and $\alpha>E_{r}$. Then there exists a unique state $(\hat{\rho}, E(\hat{\rho})) \in T\left(\rho_{l}, \alpha\right)$ satisfying $F^{-1}\left(\rho_{c}\right)<\hat{\rho}<F^{-1}\left(\rho_{r}\right)$ and $-E_{r}<E(\hat{\rho})<-E_{c}$ such that

$$
\lim _{\bar{\rho} \rightarrow \rho_{c}+} \mu^{\prime}(\bar{\rho})=-\infty,\left\{\begin{array}{l}
\mu^{\prime}(\bar{\rho})<0, \text { for } F^{-1}\left(\rho_{c}\right)<\bar{\rho}<\hat{\rho} \\
\mu^{\prime}(\bar{\rho})>0, \text { for } \hat{\rho}<\bar{\rho} \leq F^{-1}\left(\rho_{r}\right) .
\end{array}\right.
$$

So

$$
\mu(\hat{\rho})=\min _{F^{-1}\left(\rho_{c}\right) \leq \bar{\rho} \leq F^{-1}\left(\rho_{r}\right)} \mu(\bar{\rho}) .
$$

The proof of this Lemma is given in the Appendix.

The following three theorems give respectively the non-existence, the existence and uniqueness, and existence of multiple transonic shock solutions to the boundary 
value problem (1.7) and (1.8) under various conditions, for the case when $\left(\rho_{l}, \alpha\right)$ satisfies (6.36). In order to state these theorems, we set

$$
\beta=\sqrt{\alpha^{2}+2\left(H\left(\rho_{r}\right)-H\left(F\left(\rho_{l}\right)\right)\right.}, \text { when it is well defined. }
$$

Also, in the statement of the following three theorems, the functions $Y, Z, \bar{Y}, \bar{Z}, \mu$, and the state $\hat{\rho}$ are given in the analysis for subcases $2-4$. So we do not specify them in the statement of the theorems.

THEOREM 6.6 (Non-existence of transonic shock solutions).

Suppose that $\left(\rho_{l}, \alpha\right)$ satisfies (6.36). Then the boundary value problem (1.7) and (1.8) has no solution if $L<\ell\left(\left(F\left(\rho_{l}\right), \alpha\right),\left(\rho_{r}, \beta\right)\right)$, or

(a) in Subcase 1, (6.38), if $\alpha<-E_{c}$;

(b) in Subcase 2, i.e., (6.39), if $-E_{r}^{1}<\alpha<E_{r}^{0}$ or $\alpha<-E_{c}$ or $\alpha>E_{r}^{0}$ and $\ell\left(\left(\rho_{l}, \alpha\right) ;\left(F^{-1}\left(\rho_{r}\right), E_{r}^{0}\right)\right)<L<Y(\hat{\rho})$, (where and in the following $\hat{\rho}$ is given in (6.45));

(c) in Subcase 3, (6.40): if $-\bar{E}_{r}<\alpha<E_{r}$ or $\alpha<-E_{c}$;

(d) in Subcase 4, (6.41), if $-E_{c} \leq \alpha<E_{r}$ or $\alpha<-E_{r}$.

THEOREM 6.7 (Existence and Uniqueness of transonic shock solutions).

Suppose that $\left(\rho_{l}, \alpha\right)$ satisfies (6.36). Then the boundary value problem (1.7) and (1.8) has a unique transonic shock solution in the following cases:

(a) in Subcase 1, (6.38), if

$$
\alpha>-E_{c} \text { and } \ell\left(\left(F\left(\rho_{l}\right), \alpha\right) ;\left(\rho_{r}, \beta\right)\right) \leq L<+\infty ;
$$

(b) in Subcase 2, (6.39), if

$$
\alpha>E_{r}^{0} \text { and } \ell\left(\left(F\left(\rho_{l}\right), \alpha\right) ;\left(\rho_{r}, \beta\right)\right) \leq L \leq \ell\left(\left(\rho_{l}, \alpha\right) ;\left(F^{-1}\left(\rho_{r}\right), E_{r}^{0}\right)\right) ;
$$

or if $\alpha>E_{r}^{0}$, and

$$
\left\{\begin{array}{l}
\alpha>E_{r}^{0} \text { and } \\
\min \left\{Y\left(F^{-1}\left(\bar{\rho}_{r}\right)\right), Y\left(F^{-1}\left(\rho_{r}\right)\right)\right\}<L<\max \left\{Y\left(F^{-1}\left(\bar{\rho}_{r}\right)\right), Y\left(F^{-1}\left(\rho_{r}\right)\right)\right\} \\
\quad \text { when } Y\left(F^{-1}\left(\bar{\rho}_{r}\right)\right)>Y\left(F^{-1}\left(\rho_{r}\right)\right)
\end{array}\right.
$$

where the function $Y$ is given in (6.44);

(c) in Subcase 3, (6.40), if

$$
\alpha>E_{r} \text { and } \ell\left(\left(F\left(\rho_{l}\right), \alpha\right) ;\left(\rho_{r}, \beta\right)\right) \leq L \leq \ell\left(\left(\rho_{l}, \alpha\right) ;\left(F^{-1}\left(\rho_{r}\right), E_{r}\right)\right) ;
$$

or if

$$
\left\{\begin{array}{l}
\alpha>E_{r} \text { and } \\
\min \left\{\bar{Y}\left(F^{-1}\left(\bar{\rho}_{r}\right)\right), \bar{Y}\left(F^{-1}\left(\rho_{r}\right)\right)\right\}<L<\max \left\{\bar{Y}\left(F^{-1}\left(\bar{\rho}_{r}\right)\right), \bar{Y}\left(F^{-1}\left(\rho_{r}\right)\right)\right\} \\
\text { when } \bar{Y}\left(F^{-1}\left(\bar{\rho}_{r}\right)\right)>\bar{Y}\left(F^{-1}\left(\rho_{r}\right)\right)
\end{array}\right.
$$

or if

$$
\max \left\{\bar{Y}\left(F^{-1}\left(\bar{\rho}_{r}\right)\right), \bar{Y}\left(F^{-1}\left(\rho_{r}\right)\right)\right\} \leq L<+\infty ;
$$

(d) in Subcase 4, (6.41), if

$$
\alpha>E_{r} \text { and } \ell\left(\left(F\left(\rho_{l}\right), \alpha\right) ;\left(\rho_{r}, \beta\right)\right) \leq L \leq \ell\left(\left(\rho_{l}, \alpha\right) ;\left(F^{-1}\left(\rho_{r}\right), E_{r}\right)\right),
$$


or if

$$
\mu\left(F^{-1}\left(\rho_{r}\right)\right) \leq L<+\infty
$$

THEOREM 6.8 (Existence of multiple transonic shock solutions).

Suppose that $\left(\rho_{l}, \alpha\right)$ satisfies (6.36). Then the boundary value problem (1.7) and (1.8) has more than one transonic shock solutions in the following cases:

(a) In Subcase 2, (6.39), if $\alpha>E_{r}^{0}$ and $Y(\hat{\rho})<L \leq \min \left\{Y\left(F^{-1}\left(\rho_{r}^{1}\right)\right), Y\left(F^{-1}\left(\rho_{r}\right)\right)\right\}$, then there exist two and only two solutions; if $\alpha>E_{r}^{0}$ and

$$
\min \left\{Y\left(F^{-1}\left(\rho_{r}^{1}\right)\right), Y\left(F^{-1}\left(\rho_{r}\right)\right)\right\}<L<\max \left\{Y\left(F^{-1}\left(\rho_{r}^{1}\right)\right), Y\left(F^{-1}\left(\rho_{r}\right)\right)\right\}
$$

when $Y\left(F^{-1}\left(\rho_{r}^{1}\right)\right)<Y\left(F^{-1}\left(\rho_{r}\right)\right)$,

then there exist at least two transonic shock solutions;

(b) In Subcase 3, (6.40), if $\alpha>E_{r}$ and $\bar{Y}(\hat{\rho})<L \leq \min \left\{\bar{Y}\left(F^{-1}\left(\bar{\rho}_{r}\right)\right), \bar{Y}\left(F^{-1}\left(\rho_{r}\right)\right)\right\}$, then there exist two and only two solutions; if $\alpha>E_{r}$ and

$$
\begin{aligned}
& \min \left\{\bar{Y}\left(F^{-1}\left(\bar{\rho}_{r}\right)\right), \bar{Y}\left(F^{-1}\left(\rho_{r}\right)\right)\right\}<L<\max \left\{\bar{Y}\left(F^{-1}\left(\bar{\rho}_{r}\right)\right), \bar{Y}\left(F^{-1}\left(\rho_{r}\right)\right)\right\} \\
& \text { when } \bar{Y}\left(F^{-1}\left(\bar{\rho}_{r}\right)\right)<\bar{Y}\left(F^{-1}\left(\rho_{r}\right)\right),
\end{aligned}
$$

then there at least two transonic solutions;

(c) In Subcase 4, (6.41), if $\alpha>E_{r}$ and

$$
\begin{aligned}
& \min \left\{\mu\left(F^{-1}\left(\bar{\rho}_{r}\right)\right), \mu\left(F^{-1}\left(\rho_{r}\right)\right)\right\}<L<\max \left\{\mu\left(F^{-1}\left(\bar{\rho}_{r}\right)\right), \mu\left(F^{-1}\left(\rho_{r}\right)\right)\right\} \\
& \text { when } \mu\left(F^{-1}\left(\bar{\rho}_{r}\right)\right)<\mu\left(F^{-1}\left(\rho_{r}\right)\right),
\end{aligned}
$$

then there at least two transonic solutions.

Proof of Theorem 6.6. It follows from the definition of $\beta$ that $\left(\rho_{r}, \beta\right) \in$ $T\left(F\left(\rho_{l}\right), \alpha\right)$. Then it can be checked easily that the boundary value problem (1.7) and (1.8) has no solution if $L<\ell\left(\left(F\left(\rho_{l}\right), \alpha\right),\left(\rho_{r}, \beta\right)\right)$ by studying the phase portraits. The other parts in (a)-(d) can be proved by the phase portrait analysis as well (see Figures 6-9).

\section{Proof of Theorem 6.7.}

Proof. Proof of (a). In this case, it will be shown show that if $\alpha>-E_{c}$ and $\ell\left(\left(F\left(\rho_{l}\right), \alpha\right) ;\left(\rho_{r}, \beta\right)\right) \leq L<+\infty$, then there exists a unique state $\left(\rho^{*}, E^{*}\right) \in T\left(\rho_{l}, \alpha\right)$ satisfying $\rho_{\min }^{\text {out }} \leq \rho^{*} \leq \rho_{l}$ and $-E_{c}<E^{*} \leq \alpha$ and there is a constant $E_{r}$ such that

$$
\left(\rho_{r}, E_{r}\right) \in T\left(F\left(\rho^{*}\right), E^{*}\right), L=\ell\left(\left(\rho_{l}, \alpha\right) ;\left(\rho^{*}, E^{*}\right)\right)+\ell\left(\left(F\left(\rho^{*}\right), E^{*}\right) ;\left(\rho_{r}, E_{r}\right)\right),
$$

so the transonic shock location is $a=\ell\left(\left(\rho_{l}, \alpha\right) ;\left(\rho^{*}, E^{*}\right)\right)$. We prove this for $\alpha>0$. The case when $-E_{c}<\alpha \leq 0$ can be handled similarly.

In this case, we claim:

(i) If

$$
\ell\left(\left(F\left(\rho_{l}\right), \alpha\right) ;\left(\rho_{r}, \beta\right)\right) \leq L \leq \ell\left(\left(\rho_{l}, \alpha\right) ;\left(\rho_{\text {min }}^{\text {out }}, 0\right)\right)+\ell\left(\left(F\left(\rho_{\text {min }}^{\text {out }}\right), 0\right) ;\left(\rho_{r}, E_{r 1}\right)\right),
$$

where $\beta$ is given by $(6.55), E_{r 1}$ is determined by

$$
E_{r 1}=\sqrt{2\left(H\left(\rho_{r}\right)-H\left(F\left(\rho_{\text {min }}^{\text {out }}\right)\right)\right.},
$$


such that $\left(\rho_{r}, E_{r 1}\right) \in T\left(F\left(\rho_{\text {min }}^{\text {out }}\right), 0\right)$, then there exist a unique state $\left(\rho^{*}, E^{*}\right) \in T\left(\rho_{l}, \alpha\right)$ satisfying $\rho_{\text {min }}^{\text {out }} \leq \rho^{*} \leq \rho_{l}$ and $0 \leq E^{*} \leq \alpha$ and a constant $E_{r}$ such that

$$
\left(\rho_{r}, E_{r}\right) \in T\left(F\left(\rho^{*}, E^{*}\right)\right), L=\ell\left(\left(\rho_{l}, \alpha\right) ;\left(\rho^{*}, E^{*}\right)\right)+\ell\left(\left(F\left(\rho^{*}\right), E^{*}\right) ;\left(\rho_{r}, E_{r}\right)\right),
$$

(ii) If

$$
\ell\left(\left(\rho_{l}, \alpha\right) ;\left(\rho_{\text {min }}^{\text {out }}, 0\right)\right)+\ell\left(\left(F\left(\rho_{m i n}^{\text {out }}\right), 0\right) ;\left(\rho_{r}, E_{r 1}\right)\right) \leq L<+\infty,
$$

then there exist a unique state $\left(\rho^{*}, E^{*}\right) \in T\left(\rho_{\text {min }}^{\text {out }}, 0\right)$ satisfying $\rho_{\text {min }}^{\text {out }} \leq \rho^{*} \leq F^{-1}\left(\rho_{c}\right)$ and $-E_{c}<E^{*} \leq 0$ and a constant $E_{r}^{*}>0$ such that

$$
\left(\rho_{r}, E_{r}^{*}\right) \in T\left(F\left(\rho^{*}\right), E^{*}\right), L=\ell\left(\left(\rho_{l}, \alpha\right) ;\left(\rho^{*}, E^{*}\right)\right)+\ell\left(\left(F\left(\rho^{*}\right), E^{*}\right) ;\left(\rho_{r}, E_{r}^{*}\right)\right) .
$$

This will be proved by using Lemmas 4.3 and 4.4. First, if (6.57) holds, we define

$$
X(\bar{\rho})=\ell\left(\left(\rho_{l}, \alpha\right) ;(\bar{\rho}, E(\bar{\rho}))\right)+\ell\left((F(\bar{\rho}), E(\bar{\rho})) ;\left(\rho_{r}, E_{r}(\bar{\rho})\right)\right),
$$

for $(\bar{\rho}, E(\bar{\rho})) \in T\left(\rho_{l}, \alpha\right), \rho_{\text {min }}^{\text {out }} \leq \rho<\rho_{l}$ and $0<E(\bar{\rho}) \leq \alpha$. Here the meaning of $E_{r}(\bar{\rho})$ is the same as that in Lemma 4.3, i.e.

$$
E_{r}(\bar{\rho})=\sqrt{E^{2}(\bar{\rho})+2\left(H(F(\bar{\rho}))-H\left(\rho_{r}\right)\right)} .
$$

Then one can apply (4.7) in Lemma 4.3 to obtain

$$
X^{\prime}(\bar{\rho})<0, \text { for } \rho_{\text {min }}^{\text {out }} \leq \rho<\rho_{l},
$$

due to the fact that $0<\bar{\rho}<\rho_{s}, \rho_{r}>F(\bar{\rho})>\rho_{s}>\bar{\rho}, E(\bar{\rho})>0$, and $E(\bar{\rho}, t)>0$ for $\rho_{r}>$ $t>F(\bar{\rho})$ (the definition of $E(\bar{\rho}, t)$ can be found in Lemma 4.3). This proves (i).

In order to prove (ii), we set

$$
\phi(\bar{\rho})=\ell\left(\left(\rho_{\text {min }}^{\text {out }}, 0\right) ;(\bar{\rho}, E(\bar{\rho}))\right)+\ell\left((F(\bar{\rho}), E(\bar{\rho})) ;\left(\rho_{r}, E_{r}(\bar{\rho})\right)\right),
$$

for $(\bar{\rho}, E(\bar{\rho})) \in T\left(\rho_{\text {min }}^{\text {out }}, 0\right), \rho_{\text {min }}^{\text {out }}<\bar{\rho}<F^{-1}\left(\rho_{c}\right)$, and $-E_{c}<E(\bar{\rho})<0$. Here the meaning of $E_{r}(\bar{\rho})$ is the same as $(6.62)$. It follows from Lemma 4.4 that

$$
\begin{gathered}
\frac{d \phi(\bar{\rho})}{d \bar{\rho}}=\left(p^{\prime}(\bar{\rho})-\right. \\
\left.-\frac{J^{2}}{\bar{\rho}^{2}}\right) \frac{F(\bar{\rho})-\bar{\rho}}{\bar{\rho}}\left\{\frac{1}{(F(\bar{\rho})-b) E(\bar{\rho})}-\frac{b}{F(\bar{\rho})}\left[\frac{1}{\left(\rho_{r}-b\right) E_{r}(\bar{\rho})}\right.\right. \\
\left.\left.+\int_{F(\bar{\rho})}^{q(\bar{\rho})} \frac{d \rho}{(\rho-b)^{2} E_{1}(\rho, \bar{\rho})}+\int_{q(\bar{\rho})}^{\rho_{r}} \frac{d \rho}{(\rho-b)^{2} E_{2}(\rho, \bar{\rho})}\right]\right\},
\end{gathered}
$$

where

$$
\begin{gathered}
E_{1}(\rho, \bar{\rho})=-\sqrt{E^{2}(\bar{\rho})+2 \int_{F(\bar{\rho})}^{\rho} H^{\prime}(t) d t}, \\
E_{2}(\rho, \bar{\rho})=\sqrt{E^{2}(\bar{\rho})+2 \int_{F(\bar{\rho})}^{\rho} H^{\prime}(t) d t},
\end{gathered}
$$

and where $q(\bar{\rho})$ is determined by

$$
E_{1}(q(\bar{\rho}), \bar{\rho})=E_{2}(q(\bar{\rho}), \bar{\rho})=0 .
$$


It is clear that $q(\bar{\rho})<F(\bar{\rho})$ and $q(\bar{\rho})<\rho_{r}$ for $\rho_{\text {min }}^{\text {out }}<\bar{\rho}<F^{-1}\left(\rho_{c}\right)$. Moreover, $E_{1}(\rho, \rho)<$ 0 as $q(\bar{\rho})<\rho \leq F(\bar{\rho})$, so

$$
\int_{F(\bar{\rho})}^{q(\bar{\rho})} \frac{1}{(\rho-b)^{2} E_{1}(\rho, \bar{\rho})} d \rho>0 .
$$

On the other hand, $q(\rho)<\rho_{r}$ and $E_{2}(\rho, \bar{\rho})>0$ as $q(\bar{\rho})<\rho \leq \rho_{r}$, so

$$
\int_{q(\bar{\rho})}^{\rho_{r}} \frac{1}{(\rho-b)^{2} E_{2}(\rho, \bar{\rho})} d \rho>0
$$

Note that $p^{\prime}(\bar{\rho})-\frac{J^{2}}{\bar{\rho}^{2}}<0, F(\bar{\rho})>b>\bar{\rho}$, and $E(\bar{\rho})<0$ for $\rho_{\text {min }}^{\text {out }}<\bar{\rho}<F^{-1}\left(\rho_{c}\right)$. This, together with (6.69) and (6.68), yields

$$
\phi^{\prime}(\bar{\rho})>0 \text {, for } \rho_{\text {min }}^{\text {out }}<\bar{\rho}<F^{-1}\left(\rho_{c}\right) .
$$

Finally, it remains to show that

$$
\lim _{\bar{\rho} \rightarrow F^{-1}\left(\rho_{c}\right)-} \ell\left((F(\bar{\rho}), E(\bar{\rho})) ;\left(\rho_{r}, E_{r}(\bar{\rho})\right)\right)=+\infty,
$$

where $E_{r}(\bar{\rho})$ is determined by $(6.62)$. Since the trajectory $T(F(\bar{\rho}), E(\bar{\rho}))$ intersects the $\rho$-axis at $(q(\bar{\rho}), 0)$, it suffices to show that

$$
\ell((F(\bar{\rho}), E(\bar{\rho})) ;(q(\bar{\rho}), 0))=\int_{F(\bar{\rho})}^{q(\bar{\rho})} \frac{p^{\prime}(t)-\frac{J^{2}}{t^{2}}}{E_{1}(t, \bar{\rho}) t} d t \rightarrow+\infty,
$$

as $\bar{\rho} \rightarrow F^{-1}\left(\rho_{c}\right)-$, where

$$
E_{1}(t, \bar{\rho})=-\sqrt{2 \int_{q(\bar{\rho})}^{t} \frac{(s-b)\left(p^{\prime}(s)-\frac{J^{2}}{s^{2}}\right)}{s} d s}, q(\bar{\rho}) \leq t \leq F(\bar{\rho}) .
$$

In fact, as $\bar{\rho}<F^{-1}\left(\rho_{c}\right), F(\bar{\rho}) \geq q(\bar{\rho})>b$. Therefore

$$
\left|E_{1}(t, \bar{\rho})\right| \leq C \sqrt{\int_{q(\rho)}^{t}(s-b) d s}=C \sqrt{\frac{1}{2}\left((t-b)^{2}-(q(\bar{\rho})-b)^{2}\right)}, q(\bar{\rho}) \leq t \leq F(\bar{\rho}) .
$$

It follows that

$$
\ell((F(\bar{\rho}), E(\bar{\rho})) ;(q(\bar{\rho}), 0)) \geq C \int_{q(\bar{\rho})}^{F(\bar{\rho})} \frac{1}{\left|E_{1}(t, \bar{\rho})\right|} d t \geq C \int_{q(\bar{\rho})}^{F(\bar{\rho})} \frac{1}{\sqrt{\frac{1}{2}\left((t-b)^{2}-(q(\bar{\rho})-b)^{2}\right)}} .
$$

As $\bar{\rho} \rightarrow F^{-1}\left(\rho_{c}\right)-, F(\bar{\rho}) \rightarrow \rho_{c}>b, q(\bar{\rho}) \rightarrow b,(6.72)$ follows.

Proof. Proof of (b). First, we claim that if $\ell\left(\left(F\left(\rho_{l}\right), \alpha\right) ;\left(\rho_{r}, \beta\right)\right) \leq L \leq$ $\ell\left(\left(\rho_{l}, \alpha\right) ;\left(F^{-1}\left(\rho_{r}\right), E_{r}^{0}\right)\right)$, where $\beta=\sqrt{\alpha^{2}+2\left(H\left(\rho_{r}\right)-H\left(F\left(\rho_{l}\right)\right)\right.}$, then there exist a unique state $\left(\rho^{*}, E^{*}\right) \in T\left(\rho_{l}, \alpha\right)$ satisfying $F^{-1}\left(\rho_{r}\right) \leq \rho^{*} \leq \rho_{l}$ and $E_{r}^{0} \leq E^{*} \leq \alpha$ and a constant $E_{r}$ such that

$$
\left(\rho_{r}, E_{r}\right) \in T\left(F\left(\rho^{*}\right), E^{*}\right), L=\ell\left(\left(\rho_{l}, \alpha\right) ;\left(\rho^{*}, E^{*}\right)\right)+\ell\left(\left(F\left(\rho^{*}\right), E^{*}\right) ;\left(\rho_{r}, E_{r}\right)\right) .
$$


To prove this, one can define

$$
x(\bar{\rho})=\ell\left(\left(\rho_{l}, \alpha\right) ;(\bar{\rho}, E(\bar{\rho}))\right)+\ell\left((F(\bar{\rho}), E(\bar{\rho})) ;\left(\rho_{r}, E_{r}(\bar{\rho})\right)\right),
$$

for $(\bar{\rho}, E(\bar{\rho})) \in T\left(\rho_{l}, \alpha\right), F^{-1}\left(\rho_{r}\right) \leq \bar{\rho} \leq \rho_{l}$ and $E_{r}^{0} \leq E(\bar{\rho}) \leq \alpha$, where $E_{r}(\bar{\rho})$ is determined by $\left(\rho_{r}, E_{r}(\bar{\rho})\right) \in T(F(\rho), E(\bar{\rho}))$ satisfying $E_{r}^{0} \leq E_{r}(\bar{\rho}) \leq \beta$. It follows from (4.7) and (4.10) that

$$
x^{\prime}(\bar{\rho})=\left(p^{\prime}(\bar{\rho})-\frac{J^{2}}{\bar{\rho}^{2}}\right)\left(\frac{1}{\bar{\rho}}-\frac{1}{F(\bar{\rho})}\right) Q(\bar{\rho}),
$$

where

$$
Q(\bar{\rho})=\frac{1}{E(\bar{\rho})}+b \int_{F(\bar{\rho})}^{\rho_{r}} \frac{p^{\prime}(t)-\frac{J^{2}}{t^{2}}}{t E^{3}(\bar{\rho}, t)} d t
$$

Therefore,

$$
x^{\prime}(\bar{\rho})<0, F^{-1}\left(\rho_{r}\right) \leq \bar{\rho} \leq \rho_{l},
$$

since $E(\bar{\rho}) \geq E_{r}^{0}>0, F(\bar{\rho}) \leq \rho_{r}$, and $E(\bar{\rho}, t)>0$ as $F(\bar{\rho}) \leq t \leq \rho_{r}$.

Second, using Lemma 6.3, one can show that if $Y\left(F^{-1}\left(\rho_{r}^{1}\right)\right)>Y\left(F^{-1}\left(\rho_{r}\right)\right)$, then there exists a unique state $\left(\bar{\rho} *, E\left(\bar{\rho}^{*}\right)\right) \in T\left(\rho_{l}, \alpha\right)$ satisfying $\left(F^{-1}\left(\rho_{r}^{1}\right)\right)<\bar{\rho}^{*}<F^{-1}\left(\rho_{r}\right)$ such that $L=Z(\bar{\rho} *)$. So the shock location is $a=\ell\left(\left(\rho_{l}, \alpha\right) ;\left(\bar{\rho}^{*}, E\left(\bar{\rho}^{*}\right)\right)\right)$. Hence (b) is proved. if

Proof. Proof of (c). As in the proof of (a), we can show that in Subcase 3, (6.40),

$$
\alpha>E_{r}, \ell\left(\left(F\left(\rho_{l}\right), \alpha\right) ;\left(\rho_{r}, \beta\right)\right) \leq L \leq \ell\left(\left(\rho_{l}, \alpha\right) ;\left(F^{-1}\left(\rho_{r}\right), E_{r}\right)\right),
$$

then there exist a unique state $\left(\rho^{*}, E^{*}\right) \in T\left(\rho_{l}, \alpha\right)$ satisfying $F^{-1}\left(\rho_{r}\right) \leq \rho^{*} \leq \rho_{l}$ and $E_{r} \leq E^{*} \leq \alpha$ and a constant $E_{R}$ such that

$$
\left(\rho_{r}, E_{R}\right) \in T\left(F\left(\rho^{*}\right), E^{*}\right), L=\ell\left(\left(\rho_{l}, \alpha\right) ;\left(\rho^{*}, E^{*}\right)\right)+\ell\left(\left(F\left(\rho^{*}\right), E^{*}\right) ;\left(\rho_{r}, E_{R}\right)\right) .
$$

So the transonic shock location is $a=\ell\left(\left(\rho_{l}, \alpha\right) ;\left(\rho^{*}, E^{*}\right)\right)$. This finishes the first part in (c). The rest is similar to that of for (b) by using Lemma 6.4.

Proof of (d). The proof of this part is similar to that for (b).

\section{Proof of Theorem 6.8.}

Proof. Proof of (a). We consider the Subcase 2, (6.39). First, if

$$
\begin{aligned}
& \alpha>E_{r}^{0}, \\
& Y(\hat{\rho})<L \leq \min \left\{Y\left(F^{-1}\left(\rho_{r}^{1}\right)\right), Y\left(F^{-1}\left(\rho_{r}\right)\right)\right\},
\end{aligned}
$$

it then follows from (6.45) and (6.46) that there exist two and only two states $\left(\rho_{1}^{*}, E\left(\rho_{1}^{*}\right)\right) \in T\left(\rho_{l}, \alpha\right)$ and $\left(\rho_{2}^{*}, E\left(\rho_{2}^{*}\right)\right) \in T\left(\rho_{l}, \alpha\right)$ satisfying

$$
\begin{aligned}
& F^{-1}\left(\rho_{r}^{1}\right)<\rho_{1}^{*}<\hat{\rho}, \hat{\rho}<\rho_{2}^{*}<F^{-1}\left(\rho_{r}\right), \\
& E(\hat{\rho})<E\left(\rho_{1}^{*}\right)<-E_{r}^{1},-E_{r}^{0}<E\left(\rho_{2}^{*}\right)<E(\hat{\rho})
\end{aligned}
$$


such that

$$
L=Y\left(\rho_{1}^{*}\right)=Y\left(\rho_{2}^{*}\right) .
$$

In this case, there are two shock locations, i.e., $\ell\left(\left(\rho_{l}, \alpha\right) ;\left(\rho_{1}^{*}, E\left(\rho_{1}^{*}\right)\right)\right.$ and $\ell\left(\left(\rho_{l}, \alpha\right) ;\left(\rho_{2}^{*}, E\left(\rho_{2}^{*}\right)\right)\right)$.

Next, if $\alpha>E_{r}^{0}$ and

$$
\min \left\{Y\left(F^{-1}\left(\rho_{r}^{1}\right)\right), Y\left(F^{-1}\left(\rho_{r}\right)\right)\right\}<L<\max \left\{Y\left(F^{-1}\left(\rho_{r}^{1}\right)\right), Y\left(F^{-1}\left(\rho_{r}\right)\right)\right\}
$$

when $Y\left(F^{-1}\left(\rho_{r}^{1}\right)\right)<Y\left(F^{-1}\left(\rho_{r}\right)\right)$, since $Y\left(F^{-1}\left(\rho_{r}^{1}\right)\right)=Z\left(F^{-1}\left(\rho_{r}^{1}\right)\right)$, by using $(6.50)$ and (6.51), one can show that there exist two states $\left(\bar{\rho}_{1}^{*}, E\left(\bar{\rho}_{1}^{*}\right)\right) \in T\left(\rho_{l}, \alpha\right)$ and $\left(\bar{\rho}_{2}^{*}, E\left(\bar{\rho}_{2}^{*}\right)\right) \in$ $T\left(\rho_{l}, \alpha\right)$ satisfying $\left(F^{-1}\left(\rho_{r}^{1}\right)\right)<\bar{\rho}_{1}^{*}, \bar{\rho}_{2}^{*}<F^{-1}\left(\rho_{r}\right)$ such that

$$
L=Z\left(\rho_{1}^{*}\right)=Y\left(\rho_{2}^{*}\right),
$$

This finishes the proof of (a). The proofs for (b) and (c) are similar.

Next, we consider the following case.

6.2. Case 2: $\left(\rho_{l}, \alpha\right)$ is between the trajectory passing through $\left(F^{-1}(b), 0\right)$ and the subsonic part of the trajectory passing through $(b, 0)$. In this case, $\left(\rho_{l}, \alpha\right)$ satisfies:

$$
-H(b)<\frac{1}{2} \alpha^{2}-H\left(\rho_{l}\right)<-H\left(F^{-1}(b)\right), 0<\rho_{l}<\rho_{s} .
$$

The supersonic part of the trajectory passing through $(\rho, \alpha)$ intersects the line $E=0$ at $\left(\rho_{\text {min }}^{b w}, 0\right)$, the shock curve $S\left(\rho_{l}, \alpha\right)$ intersects the subsonic part of the critical trajectory passing through $(b, 0)$ at two points, denoted by $\left(\rho^{c}, E^{c}\right)$ and $\left(\rho^{c},-E^{c}\right)$.

There are four cases to consider:

$$
\begin{array}{ll}
\text { Subcase 1: } & \rho_{r}>b m, \\
\text { Subcase 2: } & F\left(\rho_{\text {min }}^{b w}\right)<\rho_{r}<b, \\
\text { Subcase3: } & \rho^{c}<\rho_{r}<F\left(\rho_{\text {min }}^{b w}\right), \\
\text { Subcase }: & \rho_{s}<\rho_{r}<\rho^{c} .
\end{array}
$$

The phase portraits for above four subcases can be found in Figures 10-13, respectively.

The analysis for Subcase 1 is simple, and Subcase 4 can be treated similarly as Subcase 3. Therefore, we give only detailed analysis for subcases 2 and 3 .

\section{Analysis on Subcase 2.}

In this case, the trajectory passing through $\left(\rho_{r}, 0\right)$ satisfying $\frac{1}{2} E^{2}-H(\rho)=-H(b)$ intersects the shock curve $S\left(\rho_{l}, \alpha\right)$ at two points, denoted by $\left(\rho^{K}, E^{K}\right)$ and $\left(\rho^{K},-E^{K}\right)$. For any state $\left(\rho_{0}, E_{0}\right)$ between the trajectory through $\left(\rho_{r}, 0\right)$ and the critical trajectory $T_{b}$ through $(b, 0)$, i.e.,

$$
-H\left(\rho_{r}\right)<\frac{1}{2} E_{0}^{2}-H\left(\rho_{0}\right)<-H(b)
$$

the trajectory through $\left(\rho_{0}, E_{0}\right)$ is also between the trajectory through $\left(\rho_{r}, 0\right)$ and the critical trajectory $T_{b}$ through $(b, 0)$, and thus intersects the line $\rho=\rho_{r}$ at two points, denoted by $\left(\rho_{r}, E_{r}\left(\rho_{0}, E_{0}\right)\right)$ and $\left(\rho_{r},-E_{r}\left(\rho_{0}, E_{0}\right)\right)$.

Analysis on Subcase 3. 
In this case, the line $\rho=\rho_{r}$ intersects the shock curve $S\left(\rho_{l}, \alpha\right)$ at two points $\left(\rho_{r}, E_{r}^{0}\right)$ and $\left(\rho_{r},-E_{r}^{0}\right)$ with $E_{r}^{0}>0$. The trajectory passing through $(b, 0)$ satisfying $\frac{1}{2} E^{2}-H(\rho)=-H(b)$ intersects the shock curve $S\left(\rho_{l}, \alpha\right)$ at two points $\left(\rho^{c}, E^{c}\right)$ and $\left(\rho^{c},-E^{c}\right)$ with $E^{c}>0$ (see Figure 12).

For $\bar{\rho} \in\left[\rho_{m i n}^{b w}, F^{-1}\left(\rho_{r}\right)\right]$, set

$$
E_{r}^{-}(\bar{\rho})=-\sqrt{E_{-}^{2}(\bar{\rho})+2\left(H\left(\rho_{r}\right)-H(F(\bar{\rho}))\right)},
$$

where

$$
E_{-}(\bar{\rho})=-\sqrt{\alpha^{2}+2\left(H(\bar{\rho})-H\left(\rho_{l}\right)\right)}
$$

satisfies $\left(\bar{\rho}, E_{-}(\bar{\rho})\right) \in T\left(\rho_{m i n}^{b w}, 0\right) \subset T\left(\rho_{l}, \alpha\right)$ and $-E_{r}^{0} \leq E_{-}(\bar{\rho}) \leq 0$. In this case, we define also

$$
\begin{gathered}
\mathfrak{Y}(\bar{\rho})=\ell\left(\left(\rho_{l}, \alpha\right) ;\left(\bar{\rho}, E_{-}(\bar{\rho})\right)\right)+\ell\left(\left(F(\bar{\rho}), E_{-}(\bar{\rho})\right) ;\left(\rho_{r}, E_{r}(\bar{\rho})\right)\right), \\
\text { for } \bar{\rho} \in\left[F^{-1}\left(\rho_{\text {min }}^{b w}\right), F^{-1}\left(\rho_{r}\right)\right],-E_{r}^{0} \leq E_{-}(\bar{\rho}) \leq 0 .
\end{gathered}
$$

For $\bar{\rho} \in\left[\rho_{\text {min }}^{b w}, F^{-1}\left(\rho_{c}\right)\right]$, set

$$
E_{r}^{-}(\bar{\rho})=-\sqrt{E_{+}^{2}(\bar{\rho})+2\left(H\left(\rho_{r}\right)-H(F(\bar{\rho}))\right)},
$$

where

$$
E_{+}(\bar{\rho})=\sqrt{\alpha^{2}+2\left(H(\bar{\rho})-H\left(\rho_{l}\right)\right)}
$$

satisfies $\left(\bar{\rho}, E_{+}(\bar{\rho})\right) \in T\left(\rho_{l}, \alpha\right)$ and $0 \leq E_{+}(\bar{\rho}) \leq E^{c}$. In this case, we define

$$
\begin{aligned}
& \mathcal{Z}(\bar{\rho})=\ell\left(\left(\rho_{l}, \alpha\right) ;\left(\bar{\rho}, E_{+}(\bar{\rho})\right)\right)+\ell\left(\left(F(\bar{\rho}), E_{+}(\bar{\rho})\right) ;\left(\rho_{r}, E_{r}^{-}(\bar{\rho})\right)\right), \\
& \text { for } \bar{\rho} \in\left[\rho_{\text {min }}^{b w}, F^{-1}\left(\rho_{c}\right)\right), 0 \leq E_{+}(\bar{\rho})<E^{c} .
\end{aligned}
$$

It is easy to see that

$$
\mathfrak{Y}\left(\rho_{\text {min }}^{b w}\right)=\mathfrak{Z}\left(\rho_{\text {min }}^{b w}\right)
$$

Then the following lemma holds.

Lemma 6.9. Suppose that $\left(\rho_{l}, \alpha\right)$ satisfies (6.78), $\rho_{r}$ satisfies (6.81), and $\alpha>E^{c}$. Then there exists a unique state $(\hat{\rho}, E(\hat{\rho})) \in T\left(\rho_{\min }^{\min }, 0\right) \subset T\left(\rho_{l}, \alpha\right)$ satisfying $\rho_{\min }^{c}<\hat{\rho}<$ $F^{-1}\left(\rho_{r}\right)$ and $-E_{r}^{0}<E(\hat{\rho})<0$ such that

$$
\mathfrak{Y}^{\prime}\left(\rho_{\text {min }}^{b w}\right)=-\infty,\left\{\begin{array}{l}
\mathfrak{Y}^{\prime}(\bar{\rho})<0, \text { for } \rho_{\text {min }}^{b w}<\bar{\rho}<\hat{\rho}, \\
\mathfrak{Y}^{\prime}(\bar{\rho})>0, \text { for } \hat{\rho}<\bar{\rho} \leq F^{-1}\left(\rho_{r}\right) .
\end{array}\right.
$$

So

$$
\mathfrak{Y}(\hat{\rho})=\min _{\rho_{\text {min }}^{b w} \leq \bar{\rho} \leq F^{-1}\left(\rho_{r}\right)} \mathfrak{Y}(\bar{\rho}) .
$$

Also

$$
\begin{aligned}
& \mathfrak{Z}^{\prime}(\bar{\rho})>0, \text { for } \rho_{\text {min }}^{b w} \leq \bar{\rho}<F^{-1}\left(\rho^{c}\right), \\
& \lim _{\bar{\rho} \rightarrow F^{-1}\left(\rho^{c}\right)} \mathfrak{Z}(\bar{\rho})=+\infty
\end{aligned}
$$


The proof of this lemma is almost the same as that for Lemma 6.3 and thus omitted.

With these preparations, we are ready to state the following well-posedness results for transonic shock solutions. We will consider only Subcases 1-3 since the results when Subcase 4 holds are completely similar to those for Subcase 3.

THEOREM 6.10. Suppose that $\left(\rho_{l}, \alpha\right)$ satisfies (6.78). Then the boundary value problem (1.7) and (1.8)

(1) has no solution if

(1a) in Subcase 1, (i) $\alpha \leq E^{c}$ or (ii)

$$
\alpha>E^{c}, L<\ell\left(\left(F\left(\rho_{l}\right), \alpha\right) ;\left(\rho_{r}, E_{r}^{\alpha}\right)\right),
$$

(where $E_{r}^{\alpha}$ is determined by $\left(\rho_{r}, E_{r}^{\alpha}\right) \in T\left(F\left(\rho_{l}\right), \alpha\right)$ );

(1b) in Subcase 2, (i) $\alpha<E^{K}$ or (ii) (6.92) holds;

(1c) in Subcase 3, (i) $\alpha<-E_{r}^{0}$ or (ii) (6.92) holds; or (iii)

$$
\left.\alpha>E^{c}, \ell\left(\left(\rho_{l}, \alpha\right) ;\left(F^{-1}\left(\rho_{r}\right), E_{r}^{0}\right)\right)<L<\mathfrak{Y}(\hat{\rho})\right),
$$

(where and in the following $\hat{\rho}$ is given in (6.90));

(2) has a unique transonic shock solution if:

(2a) in Subcase1,

$$
\alpha>E^{c}, \ell\left(\left(F\left(\rho_{l}\right), \alpha\right) ;\left(\rho_{r}, E_{r}^{\alpha}\right)\right) \leq L<\infty
$$

(2b) in Subcase 2,

$$
\alpha>E^{K}, \ell\left(\left(F\left(\rho_{l}\right), \alpha\right) ;\left(\rho_{r}, E_{r}^{\alpha}\right)\right) \leq L<+\infty
$$

(2c) in Subcase 3, (i)

$$
\alpha>E_{c}, \ell\left(\left(F\left(\rho_{l}\right), \alpha\right) ;\left(\rho_{r}, \beta\right)\right) \leq L \leq \ell\left(\left(\rho_{l}, \alpha\right) ;\left(F^{-1}\left(\rho_{r}\right), E_{r}^{0}\right)\right),
$$

where $\beta$ is determined by (6.55), or (ii)

$$
\begin{aligned}
\alpha & >E^{c}, \\
\mathfrak{Y}\left(F^{-1}\left(\rho_{\text {min }}^{b w}\right)\right) & >\mathfrak{Y}\left(F^{-1}\left(\rho_{r}\right)\right), \quad \min \left\{\mathfrak{Y}\left(\rho_{\text {min }}^{b w}\right), \mathfrak{Y}\left(F^{-1}\left(\rho_{r}\right)\right)\right\}<L<\mathfrak{Y}\left(F^{-1}\left(\rho_{\text {min }}^{b w}\right)\right),
\end{aligned}
$$

or (iii)

$$
\max \left\{\mathfrak{Y}\left(F^{-1}\left(\rho_{r}^{1}\right)\right), \mathfrak{Y}\left(F^{-1}\left(\rho_{r}\right)\right)\right\} \leq L<+\infty
$$

(3) has more than one transonic shock solutions in the following cases:

(3a) in Subcase 3, if

$$
\alpha>E^{c}, \mathfrak{Y}(\hat{\rho})<L \leq \min \left\{\mathfrak{Y}\left(\left(\rho_{m i n}^{b w}\right), \mathfrak{Y}\left(F^{-1}\left(\rho_{r}\right)\right)\right\},\right.
$$

then there exist two and only two transonic shock solutions;

(3b) in Subcase 3, there are at least two transonic shock solutions if

$$
\left\{\begin{array}{l}
\alpha>E^{c} \\
\mathfrak{Y}\left(F^{-1}\left(\rho_{m i n}^{b w}\right)\right)<\mathfrak{Y}\left(F^{-1}\left(\rho_{r}\right)\right) \\
\min \left\{\mathfrak{Y}\left(\rho_{m i n}^{b w}\right), \mathfrak{Y}\left(F^{-1}\left(\rho_{r}\right)\right)\right\}<L<\mathfrak{Y}\left(F^{-1}\left(\rho_{r}\right)\right)
\end{array}\right.
$$


(1a), (1b), and (1c) are easily seen from phase portraits (see Figure 10 for (1a), Figure 11 for (1b) and Figure 12 for (1c)).

Proof. Proof of (2a). When (6.94) holds, we claim that there exists a unique state $\left(\rho *, E\left(\rho^{*}\right)\right) \in T\left(\rho_{l}, \alpha\right)$ satisfying $F^{-1}\left(\rho^{c}\right)<\rho^{*} \leq \rho_{l}$ and $E^{c}<E\left(\rho^{*}\right) \leq \alpha$ such that

$$
L=\ell\left(\left(\rho_{l}, \alpha\right) ;\left(\rho^{*}, E\left(\rho^{*}\right)\right)\right)+\ell\left(\left(F\left(\rho^{*}\right) ; E\left(\rho^{*}\right)\right),\left(\rho_{r}, E_{r}^{*}\right)\right),
$$

where $E_{r}^{*}$ satisfies $\left(\rho_{r}, E_{r}^{*}\right) \in T\left(F\left(\rho^{*}\right), E\left(\rho^{*}\right)\right)$. This can be shown as follows: If $\rho_{r}>b$ and $\alpha>E^{c}$, for $\left(\rho, E(\rho) \in T\left(\rho_{l}, \alpha\right)\right.$ satisfying $F^{-1}\left(\rho^{c}\right)<\rho \leq \rho_{l}$, we define

$$
X(\rho)=\ell\left(\left(\rho_{l}, \alpha\right) ;(\rho, E(\rho))+\ell\left(\left(F(\rho), E(\rho),\left(\rho_{r}, E_{r}(\rho)\right)\right),\right.\right.
$$

where $E_{r}(\rho)$ satisfies $\left(\rho_{r}, E_{r}(\rho)\right) \in T(F(\rho), E(\rho))$. It follows from (4.7) that

$$
X^{\prime}(\rho)<0
$$

for $(\rho, E(\rho)) \in T\left(\rho_{l}, \alpha\right)$ satisfying $F^{-1}\left(\rho^{c}\right)<\rho \leq \rho_{l}$ and $E^{c}<E(\rho) \leq \alpha$. Moreover, just as $(6.72)$,

$$
\lim _{\rho \rightarrow F^{-1}\left(\rho^{c}\right)+} \ell\left((F(\rho), E(\rho)) ;\left(\rho_{r}, E_{r}(\rho)\right)\right) \rightarrow+\infty .
$$

This implies

$$
\lim _{\rho \rightarrow F^{-1}\left(\rho^{c}\right)+} X(\rho) \rightarrow+\infty
$$

(6.100) follows from (6.101) and (6.103). This finishes the proof of (2a).

Proof of (2b). In this case, we show that:

Proof. (2b1) If $\alpha>E^{K}$ and

$$
\ell\left(\left(F\left(\rho_{l}\right), \alpha\right) ;\left(\rho_{r}, E_{r}^{\alpha}\right)\right) \leq L \leq \ell\left(\left(\rho_{l}, \alpha\right),\left(F^{-1}\left(\rho^{K}\right), E^{K}\right)\right)+\ell\left(\left(\rho^{K}, E^{K}\right),\left(\rho_{r}, 0\right)\right),
$$

then there exists a unique state $\left(\rho^{*}, E\left(\rho^{*}\right)\right) \in T\left(\rho_{l}, \alpha\right)$ satisfying $F^{-1}\left(\rho^{K}\right) \leq \rho^{*} \leq \rho_{l}$ and $E^{K} \leq E\left(\rho^{*}\right) \leq \alpha$ such that

$$
L=\ell\left(\left(\rho_{l}, \alpha\right) ;\left(\rho^{*}, E\left(\rho^{*}\right)\right)\right)+\ell\left(\left(F\left(\rho^{*}\right), E\left(\rho^{*}\right)\right) ;\left(\rho_{r}, E_{r}^{*}\right)\right),
$$

where $E_{r}^{*}$ satisfies $\left(\rho_{r}, E_{r}^{*}\right) \in T\left(F\left(\rho^{*}\right), E\left(\rho^{*}\right)\right)$;

(2b2) If $\alpha>E^{K}$ and

$$
\ell\left(\left(\rho_{l}, \alpha\right),\left(F^{-1}\left(\rho^{K}\right), E^{K}\right)\right)+\ell\left(\left(\rho^{K}, E^{K}\right),\left(\rho_{r}, 0\right)\right) \leq L<+\infty,
$$

then there exists a unique state $\left(\rho^{*}, E\left(\rho^{*}\right)\right) \in T\left(\rho_{l}, \alpha\right)$ satisfying $F^{-1}\left(\rho^{K}\right) \leq \rho^{*} \leq \rho_{l}$ and $E^{K} \leq E\left(\rho^{*}\right)<E^{c}$ such that

$$
L=\ell\left(\left(\rho_{l}, \alpha\right) ;\left(\rho^{*}, E\left(\rho^{*}\right)\right)\right)+\ell\left(\left(F\left(\rho^{*}\right), E\left(\rho^{*}\right)\right) ;\left(\rho_{r},-E_{r}\left(\left(F\left(\rho^{*}\right), E^{*}\right)\right)\right)\right) .
$$

This can be shown as follows: For $(\rho, E(\rho)) \in T\left(\rho_{l}, \alpha\right)$ satisfying $F^{-1}\left(\rho^{K}\right)<\rho \leq \rho_{l}$ and $E^{K}<E(\rho) \leq \alpha$, we define

$$
A(\rho)=\ell\left(\left(\rho_{l}, \alpha\right) ;(\rho, E(\rho))\right)+\ell\left((F(\rho), E(\rho)) ;\left(\rho_{r}, E_{r}(\rho)\right)\right),
$$


where $E_{r}(\rho)$ satisfies $\left(\rho_{r}, E_{r}(\rho)\right) \in T(F(\rho), E(\rho))$. Then (4.7) implies that

$$
A^{\prime}(\rho)<0
$$

for $(\rho, E(\rho)) \in T\left(\rho_{l}, \alpha\right)$ satisfying $F^{-1}\left(\rho^{K}\right)<\rho \leq \rho_{l}$. This proves (2b1).

For $(\bar{\rho}, E(\bar{\rho})) \in T\left(\rho_{l}, \alpha\right)$ satisfying $F^{-1}\left(\rho^{K}\right)<\bar{\rho} \leq \rho^{c}$ and $E^{K}<E(\rho) \leq \alpha$, we define

$$
B(\bar{\rho})=\ell\left(\left(\rho_{l}, \alpha\right) ;(\bar{\rho}, E(\bar{\rho}))\right)+\ell\left((F(\bar{\rho}), E(\bar{\rho})) ;\left(\rho_{r},-E_{r}(F(\bar{\rho}), E(\bar{\rho}))\right)\right) .
$$

Due to (4.18), one can obtain

$$
\begin{aligned}
\frac{d B(\bar{\rho})}{d \bar{\rho}}=\left(p^{\prime}(\bar{\rho})-\frac{J^{2}}{\bar{\rho}^{2}}\right) & \frac{F(\bar{\rho})-\bar{\rho}}{\bar{\rho}} \cdot\left\{\frac{1}{(F(\bar{\rho})-b) E(\bar{\rho})}-\frac{b}{F(\bar{\rho})}\left[\frac{1}{\left(\rho_{r}-b\right)\left(-E_{r}(F(\bar{\rho}), E(\bar{\rho}))\right.}\right.\right. \\
& \left.\left.+\int_{F(\bar{\rho})}^{q(\bar{\rho})} \frac{d \rho}{(\rho-b)^{2} E_{1}(\rho, \bar{\rho})}+\int_{q(\bar{\rho})}^{\rho_{r}} \frac{d \rho}{(\rho-b)^{2} E_{2}(\rho, \bar{\rho})}\right]\right\}
\end{aligned}
$$

Note that $q(\bar{\rho}), E_{1}(\rho, \bar{\rho}), E_{2}(\rho, \bar{\rho})$ are the same as those in Lemma 4.4, and $\bar{E}_{r}(\bar{\rho})$ in (4.18) is the same as $-E_{r}(F(\bar{\rho}), E(\bar{\rho}))$ here. Since $F(\bar{\rho})<b, E(\bar{\rho})>0, E_{r}(F(\bar{\rho}), E(\bar{\rho}))>$ $0, \rho_{r}<b, q(\bar{\rho})>F(\bar{\rho}), E_{1}(\rho, \bar{\rho})>0, q(\bar{\rho})>\rho_{r}$, and $E_{2}(\rho, \bar{\rho})<0$, it can be verified that

$$
B^{\prime}(\bar{\rho})>0
$$

for $(\bar{\rho}, E(\bar{\rho})) \in T\left(\rho_{l}, \alpha\right)$ satisfying $F^{-1}\left(\rho^{K}\right)<\bar{\rho} \leq \rho^{c}$. Moreover,

$$
\lim _{\bar{\rho} \rightarrow F^{-1}\left(\rho^{c}\right)+} \ell\left((F(\bar{\rho}), E(\bar{\rho})) ;\left(\rho_{r},-E_{r}(F(\bar{\rho}), E(\bar{\rho}))\right)\right) \rightarrow+\infty .
$$

This implies

$$
\lim _{\rho \rightarrow F^{-1}\left(\rho^{c}\right)+} B(\bar{\rho}) \rightarrow+\infty .
$$

(6.107) follows from (6.110) and (6.112). This proves (2b2).

Proof. Proof of (3a). If (6.99) holds, using Lemma 6.9 we can show that, by the same argument as in the proof of (a) in Theorem 6.8, there exist two and only two states $\left(\rho_{1}^{*}, E\left(\rho_{1}^{*}\right)\right) \in T\left(\rho_{l}, \alpha\right)$ and $\left(\rho_{2}^{*}, E\left(\rho_{2}^{*}\right)\right) \in T\left(\rho_{l}, \alpha\right)$ satisfying $\rho_{\text {min }}^{b w}<\rho_{1}^{*}<\hat{\rho}<\rho_{2}^{*}<$ $F^{-1}\left(\rho_{r}\right), E(\hat{\rho})<E\left(\rho_{1}^{*}\right)<0$ and $-E_{r}^{0}<E\left(\rho_{2}^{*}\right)<E(\hat{\rho})$ such that

$$
L=\mathfrak{Y}\left(\rho_{1}^{*}\right)=\mathfrak{Y}\left(\rho_{2}^{*}\right) .
$$

In this case, there are two shock locations, i.e., $\ell\left(\left(\rho_{l}, \alpha\right) ;\left(\rho_{1}^{*}, E\left(\rho_{1}^{*}\right)\right)\right)$ and $\ell\left(\left(\rho_{l}, \alpha\right) ;\left(\rho_{2}^{*}, E\left(\rho_{2}^{*}\right)\right)\right)$.

Proof. Proof of (3b). Similar to the proof of (3c) in Theorem 6.8, it follows from Lemma 6.9 that there exist two states $\left(\bar{\rho}_{1}^{*}, E\left(\bar{\rho}_{1}^{*}\right)\right) \in T\left(\rho_{l}, \alpha\right)$ and $\left(\bar{\rho}_{2}^{*}, E\left(\bar{\rho}_{2}^{*}\right)\right) \in T\left(\rho_{l}, \alpha\right)$ satisfying $\rho_{\min }^{b w}<\bar{\rho}_{1}^{*}<F^{-1}\left(\rho^{c}\right)<\bar{\rho}_{2}^{*}<F^{-1}\left(\rho_{r}\right), 0 \leq E\left(\bar{\rho}_{1}^{*}\right)<E^{c}$ and $-E_{r}^{0} \leq E\left(\bar{\rho}_{2}^{*}\right) \leq 0$, such that

$$
L=\mathfrak{Z}\left(\rho_{1}^{*}\right)=\mathfrak{Y}\left(\rho_{2}^{*}\right)
$$

which completes the proof of $(3 \mathrm{~b})$. 
6.3. The case when $\left(\rho_{l}, \alpha\right)$ is inside subsonic part of the trajectory passing through $(b, 0)$. In this case, $\left(\rho_{l}, \alpha\right)$ satisfies:

$$
\frac{1}{2} \alpha^{2}-H\left(\rho_{l}\right)<-H(b), 0<\rho_{l}<\rho_{s} .
$$

The curve

$$
\frac{1}{2} E^{2}-H(\rho)=\frac{1}{2} \alpha^{2}-H\left(\rho_{l}\right)
$$

which is the trajectory passing through $\left(\rho_{l}, \alpha\right)$, intersects the line $E=0$ at $\left(\rho_{\text {min }}^{\text {in }}, 0\right)$ and $\left(\rho_{\max }, 0\right)$ satisfying

$$
H\left(\rho_{\min }^{i n}\right)=H\left(\rho_{\max }\right)=H\left(\rho_{l}\right)-\frac{1}{2} \alpha^{2}, \rho_{\min }^{\text {in }}<\rho_{s}<\rho_{\max } .
$$

The curve (6.115) is a closed curve, lying inside the critical trajectory through $(b, 0)$. The shock curve $S\left(\rho_{l}, \alpha\right)$ lies inside the subsonic part of the curve (6.115), by Lemma 6.2 (see Figure 14).

It is follows easily from the phase portrait that

Theorem 6.11. Case for $\rho_{r}>\rho_{\max }$.

Suppose that $\left(\rho_{l}, \alpha\right)$ satisfies (6.114). If $\rho_{r}>\rho_{\max }$, then the boundary value problem (1.7) and (1.8) does not have a solution for any $L$ (see Figure 14).

Next, we turn to the case when $F\left(\rho_{\min }^{\text {in }}\right)<\rho_{r}<\rho_{\max }$. In this case, the trajectory though the point $\left(F\left(\rho_{\text {min }}^{\text {in }}\right), 0\right)$ satisfying $\frac{1}{2} E^{2}-H(\rho)=-H\left(\rho_{\text {min }}^{\text {in }}\right)$ intersects the shock curve $S\left(\rho_{l}, \alpha\right)$ at two pints, denoted by $\left(\rho_{K}, E_{K}\right)$ and $\left(\rho_{K},-E_{K}\right)$ with $E_{K}>0$ (see Figure 15). Then the following theorem holds.

THEOREM 6.12. Case for $F\left(\rho_{\min }^{\text {in }}\right)<\rho_{r}<\rho_{\max }$.

Assume that $\left(\rho_{l}, \alpha\right)$ satisfies (6.114). Then it holds that

(1) If $\alpha>E_{K}$, then

(1a) For

$$
L<\ell\left(\left(F\left(\rho_{l}\right), \alpha\right) ;\left(\rho_{r}, E_{r}^{\alpha}\right)\right),
$$

where $E_{r}^{\alpha}$ is determined by $\left(\rho_{r}, E_{r}^{\alpha}\right) \in T\left(F\left(\rho_{l}\right), \alpha\right)$ satisfying $E_{r}^{\alpha}>0$, the boundary value problem (1.7) and (1.8) does not have a solution with a single transonic shock;

(1b) For

$$
\left.\ell\left(\left(F\left(\rho_{l}\right), \alpha\right) ;\left(\rho_{r}, E_{r}^{\alpha}\right)\right) \leq L \leq \ell\left(\left(\rho_{l}\right), \alpha\right) ;\left(F^{-1}\left(\rho_{K}\right), E_{K}\right)\right)+\ell\left(\left(\rho_{K}, E_{K}\right) ;\left(\rho_{r}, 0\right)\right),
$$

there exists a unique state $\left(\rho *, E\left(\rho^{*}\right)\right) \in T\left(\rho_{l}, \alpha\right)$ satisfying $F^{-1}\left(\rho_{K}\right) \leq \rho^{*} \leq \rho_{l}$ and $E_{K} \leq E\left(\rho^{*}\right) \leq \alpha$ such that

$$
L=\ell\left(\left(\rho_{l}, \alpha\right) ;\left(\rho^{*}, E\left(\rho^{*}\right)\right)\right)+\ell\left(\left(F\left(\rho^{*}\right), E\left(\rho^{*}\right),\left(\rho_{r}, E_{r}^{*}\right)\right)\right),
$$

where $E_{r}^{*}$ satisfies $\left(\rho_{r}, E_{r}^{*}\right) \in T\left(F\left(\rho^{*}\right), E\left(\rho^{*}\right)\right)$ and $E_{r}^{*} \geq 0$;

(1c) In the case that

$$
\ell\left(\left(\rho_{l}, \alpha\right) ;\left(F^{-1}\left(\rho_{K}\right), E_{K}\right)\right)+\ell\left(\left(\rho_{K}, E_{K}\right) ;\left(\rho_{r}, 0\right)\right) \leq L \leq \ell\left(\left(F\left(\rho_{l}\right), \alpha\right) ;\left(\rho_{r},-E_{r}^{\alpha}\right)\right),
$$

where $E_{r}^{\alpha}$ is determined as in 1a), there exists a unique state $\left(\rho *, E\left(\rho^{*}\right)\right) \in T\left(\rho_{l}, \alpha\right)$ satisfying $F^{-1}\left(\rho_{K}\right) \leq \rho^{*} \leq \rho_{l}$ and $E_{K} \leq E\left(\rho^{*}\right) \leq \alpha$ such that

$$
L=\ell\left(\left(\rho_{l}, \alpha\right) ;\left(\rho^{*}, E\left(\rho^{*}\right)\right)\right)+\ell\left(\left(F\left(\rho^{*}\right), E\left(\rho^{*}\right)\right) ;\left(\rho_{r},-E_{r}^{*}\right)\right),
$$


where $E_{r}^{*}$ satisfies $\left(\rho_{r}, E_{r}^{*}\right) \in T\left(F\left(\rho^{*}\right), E\left(\rho^{*}\right)\right)$ and $E_{r}^{*} \geq 0$.

(2) If $\alpha<E_{K}$, the boundary value problem (1.7) and (1.8) does not have solutions with a single transonic shock.

The proof of this theorem is similar to those in Subsection 6.2, so we omit it.

The case $\rho_{s}<\rho_{r}<F\left(\rho_{m i n}^{i n}\right)$ can be handled in a similar way as for the case of $F\left(\rho_{\min }^{\text {in }}\right)<\rho_{r}<\rho_{\max }$. So we omit the details of the treatments.

7. Appendix We prove Lemmas 6.3 and 6.5 in this appendix.

Proof of Lemma 6.3.

Proof. We prove (6.45) first. Note that

$$
Y(\bar{\rho})=\ell\left(\left(\rho_{l}, \alpha\right) ;\left(\rho_{\text {min }}^{\text {out }}, 0\right)\right)+X(\bar{\rho}),
$$

where

$$
X(\bar{\rho})=\ell\left(\left(\rho_{\text {min }}^{\text {out }}, 0\right) ;(\bar{\rho}, E(\bar{\rho}))\right)+\ell\left((F(\bar{\rho}), E(\bar{\rho})) ;\left(\rho_{r},-E_{r}(\bar{\rho})\right)\right),
$$

for $\bar{\rho} \in\left[F^{-1}\left(\rho_{r}^{1}\right), F^{-1}\left(\rho_{r}\right)\right)$. So

$$
Y^{\prime}(\bar{\rho})=X^{\prime}(\bar{\rho}) .
$$

Applying (4.7) in Lemma 4.3 leads to

$$
\begin{aligned}
Y^{\prime}\left(F^{-1}\left(\rho_{r}\right)\right) & =X^{\prime}\left(F^{-1}\left(\rho_{r}\right)\right) \\
& =\left(p^{\prime}\left(F^{-1}\left(\rho_{r}\right)\right)-\frac{J^{2}}{\left(F^{-1}\left(\rho_{r}\right)\right)^{2}}\right)\left(\frac{1}{F^{-1}\left(\rho_{r}\right)}-\frac{1}{\rho_{r}}\right) \frac{1}{E\left(F^{-1}\left(\rho_{r}\right)\right)} .
\end{aligned}
$$

Since $E\left(F^{-1}\left(\rho_{r}\right)\right)<0, p^{\prime}\left(F^{-1}\left(\rho_{r}\right)\right)-\frac{J^{2}}{\left(F^{-1}\left(\rho_{r}\right)\right)^{2}}<0$ and $F^{-1}\left(\rho_{r}\right)<\rho_{r}$,

$$
Y^{\prime}\left(F^{-1}\left(\rho_{r}\right)\right)>0 .
$$

Again, by (4.7), one has

$$
\begin{aligned}
& Y^{\prime}\left(F^{-1}\left(\rho_{r}^{1}\right)\right) \\
= & X^{\prime}\left(F^{-1}\left(\rho_{r}^{1}\right)\right) \\
= & \left(p^{\prime}\left(F^{-1}\left(\rho_{r}^{1}\right)\right)-\frac{J^{2}}{\left(F^{-1}\left(\rho_{r}^{1}\right)\right)^{2}}\right)\left(\frac{1}{F^{-1}\left(\rho_{r}^{1}\right)}-\frac{1}{\rho_{r}^{1}}\right) Q\left(F^{-1}\left(\rho_{r}^{1}\right)\right),
\end{aligned}
$$

where

$$
\begin{gathered}
Q\left(F^{-1}\left(\rho_{r}^{1}\right)\right)=\frac{1}{E\left(F^{-1}\left(\rho_{r}^{1}\right)\right)}+b \int_{\rho_{r}^{1}}^{\rho_{r}} \frac{p^{\prime}(t)-\frac{J^{2}}{t^{2}}}{t E^{3}\left(F^{-1}\left(\rho_{r}^{1}\right), t\right)} d t, \\
E\left(F^{-1}\left(\rho_{r}^{1}\right), t\right)=-\sqrt{E^{2}\left(F^{-1}\left(\rho_{r}^{1}\right)\right)+2\left(H(t)-H\left(\rho_{r}^{1}\right)\right)}, \rho_{r} \leq t \leq \rho_{r}^{1} .
\end{gathered}
$$

Note that

$$
-\infty<E\left(F^{-1}\left(\rho_{r}^{1}\right)\right)<0 .
$$

We now claim that

$$
\int_{\rho_{r}^{1}}^{\rho_{r}} \frac{p^{\prime}(t)-\frac{J^{2}}{t^{2}}}{t E^{3}\left(F^{-1}\left(\rho_{r}^{1}\right), t\right)} d t=+\infty
$$


This can be shown as follows. Let

$$
g(t)=E^{2}\left(F^{-1}\left(\rho_{r}^{1}\right), t\right), \rho_{r} \leq t \leq \rho_{r}^{1} .
$$

Then

$$
\frac{1}{2} g(t)-H(t)=\frac{1}{2} E^{2}\left(F^{-1}\left(\rho_{r}^{1}\right)\right)-H\left(\rho_{r}^{1}\right), \rho_{r} \leq t \leq \rho_{r}^{1}
$$

Therefore,

$$
g^{\prime}(t)=2 H^{\prime}(t)=2\left(1-\frac{b}{t}\right)\left(p^{\prime}(t)-\frac{J^{2}}{t^{2}}\right), \rho_{r} \leq t \leq \rho_{r}^{1}
$$

Since $\rho_{r}^{1}>\rho_{r}>b>\rho_{s}$, there exist positive constants $C_{1}$ and $C_{2}$ such that

$$
C_{1} \leq g^{\prime}(t) \leq C_{2}, \rho_{r} \leq t \leq \rho_{r}^{1}
$$

Since $g\left(\rho_{r}\right)=0$, it holds that

$$
g(t)=O\left(\left|t-\rho_{r}\right|\right)
$$

for small $\left|t-\rho_{r}\right|$. This implies that

$$
E\left(F^{-1}\left(\rho_{r}^{1}\right), t\right)=O\left(\left|t-\rho_{r}\right|^{1 / 2}\right)
$$

for small $\left|t-\rho_{r}\right|$. (7.6) now follows since $\rho_{r}^{1}>\rho_{r}$ and $E\left(F^{-1}\left(\rho_{r}^{1}\right), t\right)<0$ for $\rho_{r} \leq t \leq \rho_{r}^{1}$. As a consequence of (7.3)-(7.6), one has

$$
Y^{\prime}\left(F^{-1}\left(\rho_{r}^{1}\right)\right)=-\infty
$$

In view of $(7.2)$ and $(7.8), Y^{\prime}(\bar{\rho})$ changes the sign in the interval $\left[F^{-1}\left(\rho_{r}^{1}\right), F^{-1}\left(\rho_{r}\right)\right]$. Since

$$
\operatorname{sign} Q(\bar{\rho})=-\operatorname{sign} X^{\prime}(\bar{\rho})=-\operatorname{sign} Y^{\prime}(\bar{\rho})
$$

for $\bar{\rho} \in\left[F^{-1}\left(\rho_{r}^{1}\right), F^{-1}\left(\rho_{r}\right)\right]$ (where $Q(\bar{\rho})$ is defined in $(4.10)$ ), $Q(\bar{\rho})$ changes the sign in the interval $\left[F^{-1}\left(\rho_{r}^{1}\right), F^{-1}\left(\rho_{r}\right)\right]$. Suppose that

$$
Q(\hat{\rho})=X^{\prime}(\hat{\rho})=Y^{\prime}(\hat{\rho})=0,
$$

for some $\hat{\rho} \in\left[F^{-1}\left(\rho_{r}^{1}\right), F^{-1}\left(\rho_{r}\right)\right]$. By (4.7) and (4.10), one gets

$$
Q(\hat{\rho})=\frac{1}{E(\hat{\rho})}+b \int_{F(\hat{\rho})}^{\rho_{r}} \frac{p^{\prime}(t)-\frac{J^{2}}{t^{2}}}{t E^{3}(\hat{\rho}, t)} d t=0 .
$$

This, together with (4.10), gives

$$
\begin{aligned}
& \frac{Q^{\prime}(\hat{\rho})}{p^{\prime}(\hat{\rho})-\frac{J^{2}}{\hat{\rho}^{2}}} \\
= & \frac{1}{E^{3}(\hat{\rho})}\left(\frac{b}{\hat{\rho}}-\frac{b}{F(\hat{\rho})}-1\right)+3 b^{2}\left(\frac{1}{\hat{\rho}}-\frac{1}{F(\hat{\rho})}\right) \int_{F(\hat{\rho})}^{\rho_{r}} \frac{p^{\prime}(t)-\frac{J^{2}}{t^{2}}}{t E^{5}(\hat{\rho}, t)} d t \\
= & b^{2}\left(\frac{1}{\hat{\rho}}-\frac{1}{F(\hat{\rho})}\right) \int_{F(\hat{\rho})}^{\rho_{r}} \frac{p^{\prime}(t)-\frac{J^{2}}{t^{2}}}{t E^{3}(\hat{\rho}, t)}\left(\frac{3}{E^{2}(\hat{\rho}, t)}-\frac{1}{E^{2}(\hat{\rho})}\right) d t-\frac{1}{E^{3}(\hat{\rho})} .
\end{aligned}
$$


Since $\rho_{r}<F(\hat{\rho}), E^{2}(\hat{\rho}, t)<E^{2}(\hat{\rho})$ for $\rho_{r} \leq t<F(\hat{\rho}), E(\hat{\rho})<0, F(\hat{\rho})>\hat{\rho}$ and $p^{\prime}(\hat{\rho})-\frac{J^{2}}{\hat{\rho}^{2}}<$ $0\left(\hat{\rho}<\rho_{s}\right),(7.11)$ implies

$$
Q^{\prime}(\hat{\rho})<0
$$

It follows from (7.2), (7.8), and (4.7) that

$Q\left(F^{-1}\left(\rho_{r}\right)\right)<0, Q\left(F^{-1}\left(\rho_{r}^{1}\right)\right)=+\infty, Q^{\prime}(\hat{\rho})<0$ as $Q(\hat{\rho})=0$ for $\hat{\rho} \in\left[F^{-1}\left(\rho_{r}^{1}\right), F^{-1}\left(\rho_{r}\right)\right]$.

Therefore, $Q(\bar{\rho})$ only changes the sign once for $\bar{\rho} \in\left[F^{-1}\left(\rho_{r}^{1}\right), F^{-1}\left(\rho_{r}\right)\right]$ at $\bar{\rho}=\hat{\rho}$ where $Q(\hat{\rho})=0$. Hence, we conclude that

$$
Q\left(F^{-1}\left(\rho_{r}^{1}\right)\right)=+\infty,\left\{\begin{array}{l}
Q(\bar{\rho})>0 \text { as } F^{-1}\left(\rho_{r}^{1}\right)<\bar{\rho}<\hat{\rho}, \\
Q(\hat{\rho})=0, \\
Q(\bar{\rho})<0 \text { as } \hat{\rho}<\bar{\rho} \leq \hat{\rho}, F^{-1}\left(\rho_{r}\right) .
\end{array}\right.
$$

This proves (6.45) and (6.46) in view of (4.7).

$\square$ Proof of Lemma 6.5.

Proof. We will show only

$$
\lim _{\bar{\rho} \rightarrow \rho_{c}+} \mu^{\prime}(\bar{\rho})=-\infty
$$

in (6.53). The rest of the proof is almost the same as that for Lemma 6.3.

For any $\bar{\rho} \in\left(F^{-1}\left(\rho_{c}\right), F^{-1}\left(\rho_{r}\right)\right),(4.7)$ and (4.8) imply that

$$
\mu^{\prime}(\bar{\rho})=\left(p^{\prime}(\bar{\rho})-\frac{J^{2}}{\bar{\rho}^{2}}\right)\left(\frac{1}{\bar{\rho}}-\frac{1}{F(\bar{\rho})}\right) Q(\bar{\rho}),
$$

where

$$
Q(\bar{\rho})=\frac{1}{E(\bar{\rho})}+b \int_{F(\bar{\rho})}^{\rho_{r}} \frac{p^{\prime}(t)-\frac{J^{2}}{t^{2}}}{t E^{3}(\bar{\rho}, t)} d t,
$$

with $-E_{r}<E(\bar{\rho})<-E_{c}$. The meaning of $E(\bar{\rho}, t)$ is given in (4.9). We now show that

$$
\lim _{\bar{\rho} \rightarrow F^{-1}\left(\rho_{c}\right)+} Q(\bar{\rho})=+\infty .
$$

This is equivalent to

$$
\int_{F(\bar{\rho})}^{\rho_{r}} \frac{p^{\prime}(t)-\frac{J^{2}}{t^{2}}}{t E^{3}(\bar{\rho}, t)} d t=+\infty
$$

Since $E(\bar{\rho}, t)<0, p^{\prime}(t)-\frac{J^{2}}{t^{2}}>0$, and $F(\bar{\rho})>b>\rho_{r}$ for $F^{-1}\left(\rho_{c}\right)<\bar{\rho}<F^{-1}\left(\rho_{r}\right), \rho_{r} \leq t \leq$ $F(\bar{\rho})$, it holds that

$$
\int_{F(\bar{\rho})}^{\rho_{r}} \frac{p^{\prime}(t)-\frac{J^{2}}{t^{2}}}{t E^{3}(\bar{\rho}, t)} d t \geq \int_{F(\bar{\rho})}^{b} \frac{p^{\prime}(t)-\frac{J^{2}}{t^{2}}}{t E^{3}(\bar{\rho}, t)} d t
$$

Let

$$
g(\bar{\rho}, t)=E^{2}(\bar{\rho}, t), \text { for } b \leq t \leq F(\bar{\rho}) .
$$

Then we have

$$
\frac{1}{2} g(\bar{\rho}, t)-H(t)=C(\bar{\rho})
$$



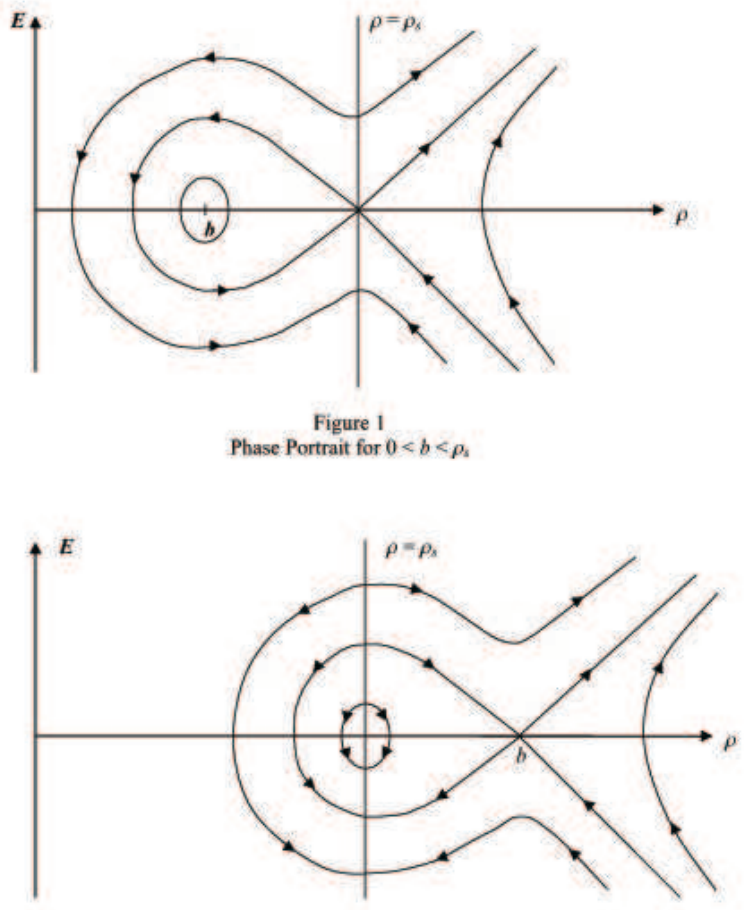

Figure 2

Phase Portrait of $b>p_{s}$

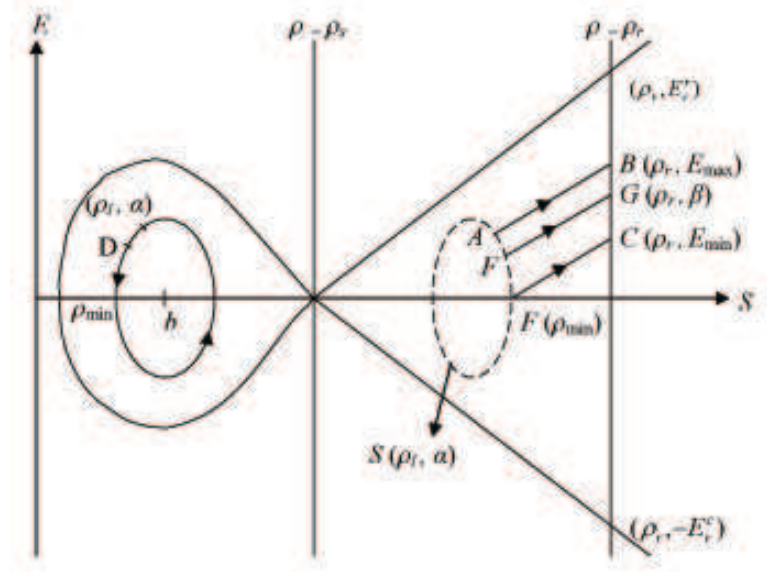

$A:\left(F\left(\rho_{i}\right), a\right), \quad B:\left(\rho_{n}, E_{\max }\right), \quad C:\left(\rho_{n}, E_{\min }\right)$

D: $\left(\rho^{*}, E^{*}\right), \quad F:\left(F\left(\rho^{*}\right), E^{*}\right)$

Figure 3

where $C(\bar{\rho})$ is a quantity depending only on $\bar{\rho}$ but not on $t$. Therefore,

$$
\frac{\partial g(\bar{\rho}, t)}{\partial t}=2 H^{\prime}(t)=2\left(1-\frac{b}{t}\right)\left(p^{\prime}(t)-\frac{J^{2}}{t^{2}}\right) .
$$




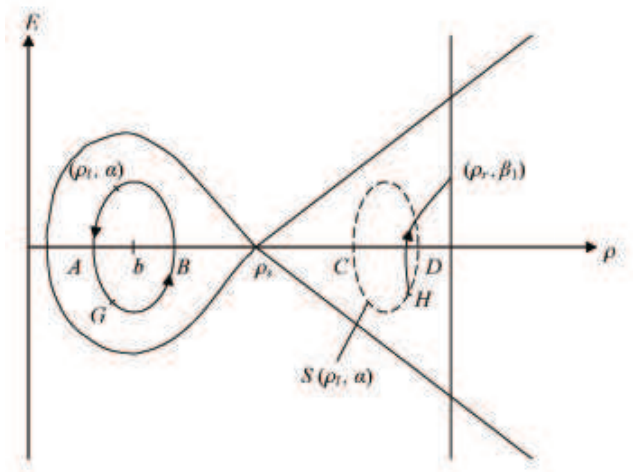

$$
A:\left(\rho_{\min }, 0\right), \quad B:\left(\rho_{\max }, 0\right), \quad C:\left(F\left(\rho_{\max }\right), 0\right),
$$

Figure 4

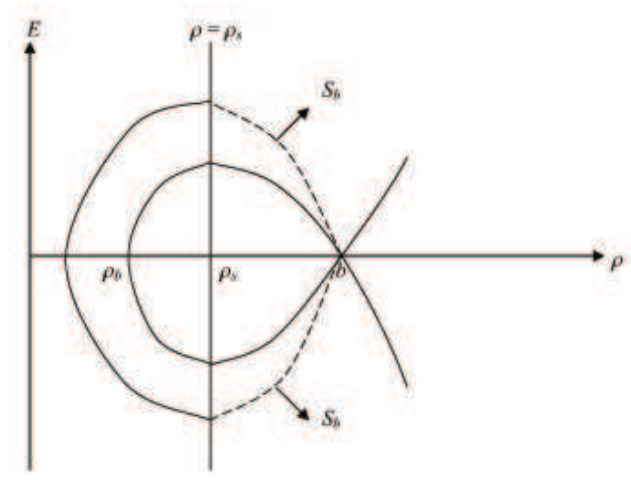

The dotted curve is $S_{i,}$

Figure 5

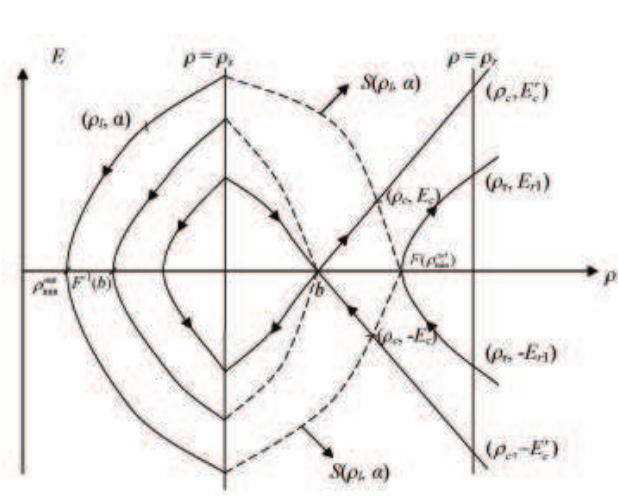

Figure 6

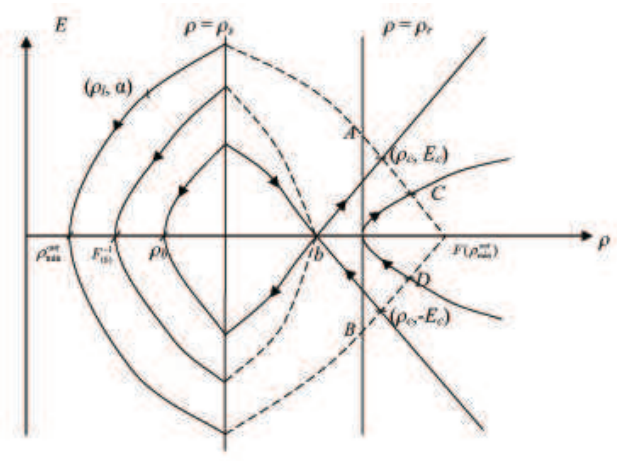

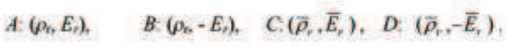

Figure 8

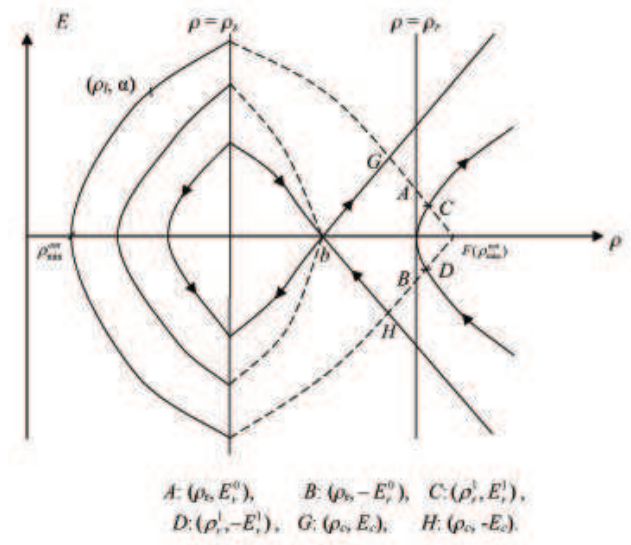

Figure ?

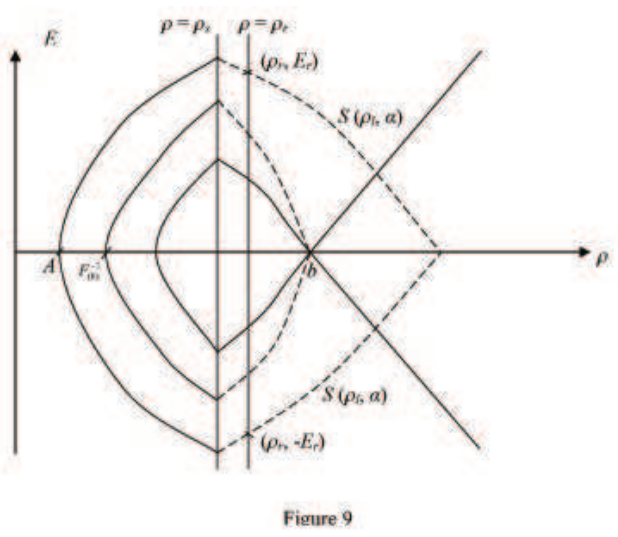



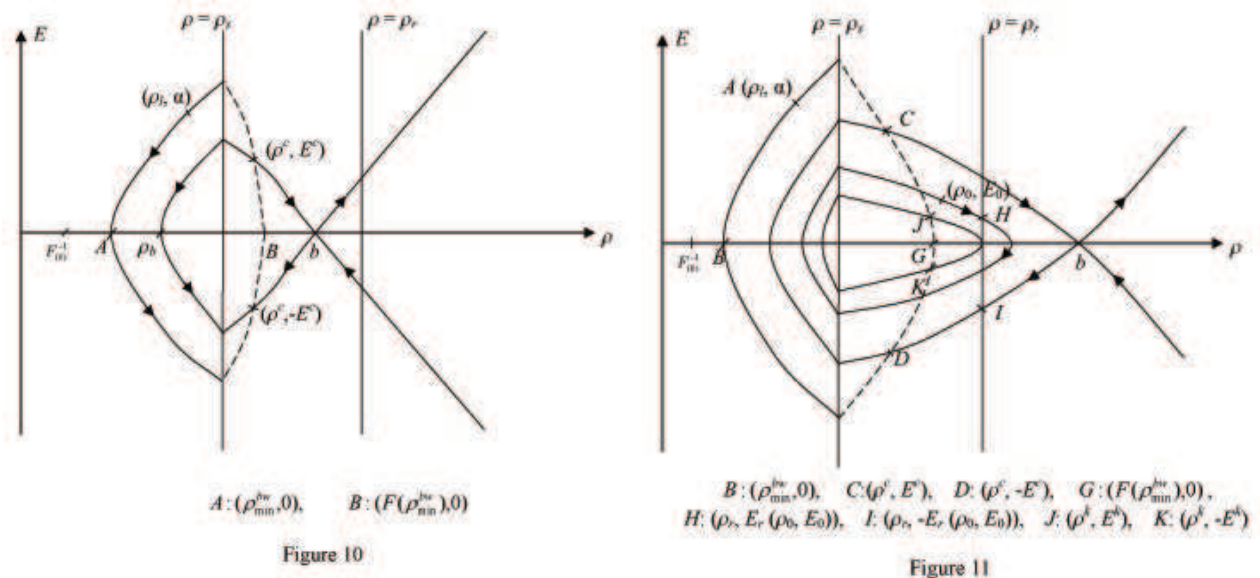

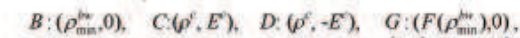

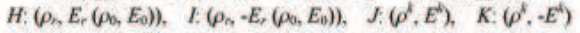

Figure 11
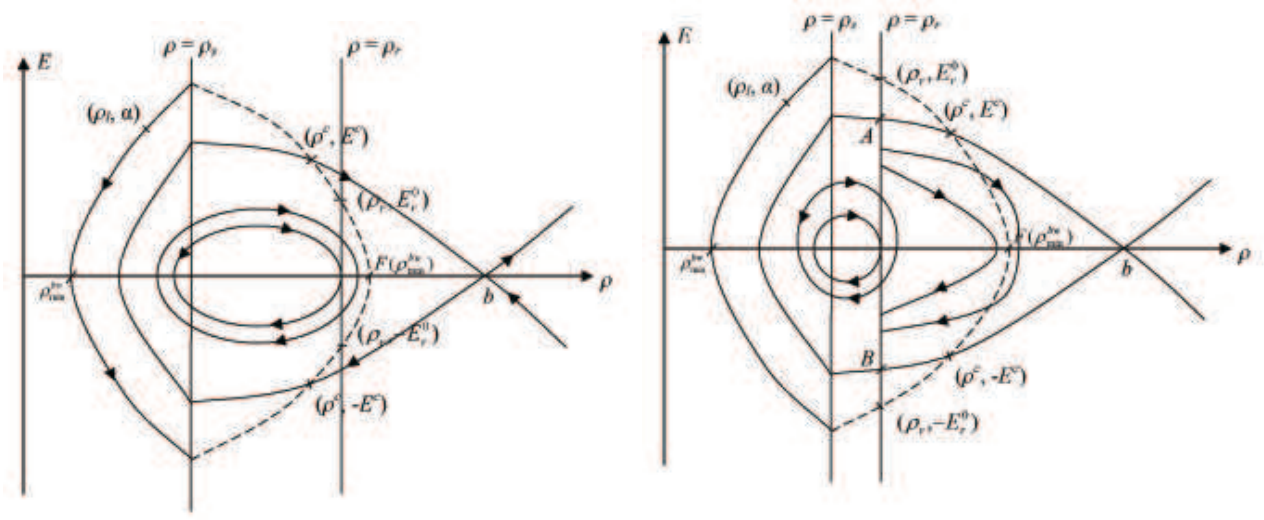

Figure 12

$A:\left(\rho_{,},-E_{,}^{\prime}\right), \quad B:\left(\rho_{r},-E_{,}^{\prime}\right)$

Figure 13

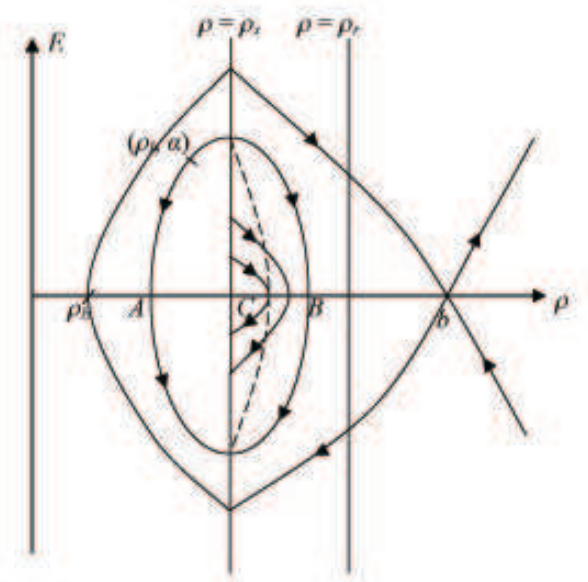

$A:\left(\rho_{\operatorname{man}}^{n}, 0\right), \quad B:\left(\rho_{\max }, 0\right), \quad C:\left(F\left(\rho_{\operatorname{mat}}^{n}\right), 0\right)$

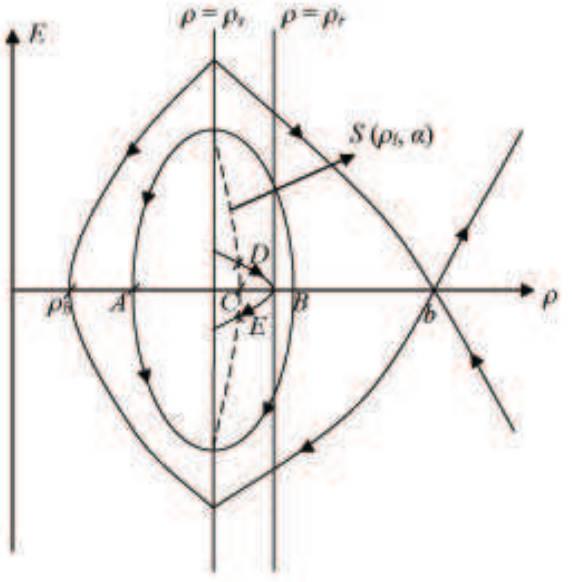

$A:\left(\rho_{\min }^{n}, 0\right), \quad B:\left(\rho_{\max }, 0\right), \quad C \div\left(F\left(\rho_{\min }^{n}\right), 0\right)$ $D:\left(\rho_{k}, E_{k}\right), \quad E:\left(\rho_{k},-E_{k}\right)$

Figure 15 
Thus

$$
\left.\frac{\partial g(\bar{\rho}, t)}{\partial t}\right|_{t=b}=0
$$

On the other hand,

$$
\lim _{\bar{\rho} \rightarrow F^{-1}\left(\rho_{c}\right)+, t \rightarrow b+} g(\bar{\rho}, t)=0 .
$$

It follows from (7.14) and (7.15) that

$$
g(\bar{\rho}, t)=o(|t-b|)
$$

as $|t-b|$ is small. Thus, (7.12) follows from (7.13) and (7.16).

Acknowledgments. This work was initiated when Luo visited Institute of Mathematical Sciences, Chinese University of Hong Kong. Luo's research was partially supported by NSF grants DMS-0742834 (continuing as DMS-0839864). Xin's research was partially supported by Hong Kong RGC Earmarked Research Grants CUHK 4040/06p, CUHK 4042/08p, the RGC Central Allocation Grant CA05/06.SC01, and a Focus Area Grant at the Chinese University of Hong Kong. The authors would like to thank Dr. Chunjing Xie for some useful discussions.

\section{REFERENCES}

[1] U.M. Asher, P.A. Markowich, P. Pietra, and C. Schmeiser, A phase plane analysis of transonic solutions for the hydrodynamic semiconductor model, Math. Mod. Meth. Appl. Sci., 1(3), 347-376, 1991.

[2] D.P. Chen, R.S. Eisenberg, J.W. Jerome, and C.W. Shu, A hydrodynamic model of temperature change in open ionic channels, Biophys. J. 69, 2304-2322, 1995.

[3] G.Q. Chen and M. Feldman, Multidimensional transonic shocks and free boundary problems for nonlinear equations of mixed type, J. AMS., 16(3), 461-494, 2003.

[4] P. Embid, J. Goodman, and A. Majda, Multiple steady states for 1-D transonic flow, SIAM J. Sci. Statist. Comput., 5(1), 21-41, 1984.

[5] P. Degond and P.A. Markowich, A steady state potential flow model for semiconductors, Ann. Mat. Pura Appl., 4(165), 87-98, 1993.

[6] I.M. Gamba, Sharp uniform bounds for steady potential fluid-Poisson systems, Proc. Roy. Soc. Edinburgh Sect., A127(3), 479-516, 1997.

[7] I.M. Gamba and C.S. Morawetz, A viscous approximation for a 2-D steady semiconductor or transonic gas dynamic flow: Existence theorem for potential flow, Commun. Pure Appl. Math., 49(10), 999-1049, 1996.

[8] I.M. Gamba, Stationary transonic solutions of a one-dimensional hydrodynamic model for semiconductors, Commun. Part. Diff. Equ., 17, 3-4, 553-577, 1992.

[9] S. Ha and T. Yang, $L^{1}$ stability for systems of hyperbolic conservation laws with a resonant moving source, SIAM J. Math. Anal., 34(5), 1226-1251, 2003.

[10] W.C. Lien, Hyperbolic conservation laws with a moving source, Commun. Pure Appl. Math., 52(9), 1075-1098, 1999.

[11] T.P. Liu, Nonlinear stability and instability of transonic gas flows through a nozzle, Commun. Math. Phys., 83(2), 243-260, 1982.

[12] T. Luo, J. Rauch, C. Xie, and Z. Xin, Stability of Transonic Shock Solutions for OneDimensional Euler-Poisson Equations, preprint, arXiv:1008.0378, 2010.

[13] P.A. Markowich, On steady state Euler-Poisson models for semiconductors, Z. Angew. Math. Phys., 42(3), 389-407, 1991.

[14] M.D. Rosini, Stability of transonic strong shock waves for the one-dimensional hydrodynamic model for semiconductors, J. Diff. Equ., 199(2), 326-351, 2004.

[15] M.D. Rosini, A phase analysis of transonic solutions for the hydrodynamic semiconductor model, Quart. Appl. Math., 63(2), 251-268, 2005.

[16] Z. Xin and H. Yin, Transonic shock in a nozzle I, 2-D case, Commun. Pure Appl. Math., 58(8), 999-1050, 2005. 